Fall 2012

\title{
Same sign dimuon search for heavy majorana mass neutrinos at the CMS experiment at Cern and design studies of a quartz plate calorimeter prototype
}

Warren James Clarida

University of Iowa

\section{Copyright 2012 Warren Clarida}

This dissertation is available at Iowa Research Online: http://ir.uiowa.edu/etd/3441

\section{Recommended Citation}

Clarida, Warren James. "Same sign dimuon search for heavy majorana mass neutrinos at the CMS experiment at Cern and design studies of a quartz plate calorimeter prototype." PhD (Doctor of Philosophy) thesis, University of Iowa, 2012.

http://ir.uiowa.edu/etd/3441.

Follow this and additional works at: http://ir.uiowa.edu/etd

Part of the Physics Commons 


\title{
SAME SIGN DIMUON SEARCH FOR HEAVY MAJORANA MASS NEUTRINOS AT THE CMS EXPERIMENT AT CERN AND DESIGN STUDIES OF A QUARTZ PLATE CALORIMETER PROTOTYPE
}

\author{
by
}

Warren James Clarida

\author{
$\underline{\text { An Abstract }}$ \\ Of a thesis submitted in partial fulfillment of the \\ requirements for the Doctor of Philosophy \\ degree in Physics in the \\ Graduate College of The \\ University of Iowa
}

December 2012

Thesis Supervisor: Professor Yasar Onel 


\begin{abstract}
This paper consists of two studies: the results of a search for heavy Majorana neutrinos $(\mathrm{N})$ using an event signature defined by two like-sign charged muons and two jets, and the results from studies of a prototype quartz plate calorimeter. The data in the Majorana search correspond to an integrated luminosity of $5.0 \mathrm{fb}^{-1}$ of pp collisions at a centre-of-mass energy of $7 \mathrm{TeV}$ collected with the CMS detector at the Large Hadron Collider. No excess of events are observed beyond the expected standard model background and therefore upper limits are set on the square of the mixing element, $\left|V_{\mu N}\right|^{2}$ as a function of Majorana neutrino mass. These are the first direct upper limits on the heavy Majorana-neutrino mixing for $m_{N}>90 \mathrm{GeV}$. The second part of this thesis is the results of performance tests of a 20-layer quartz plate calorimeter prototype. The calorimeter prototype was tested at the CERN $\mathrm{H} 2$ area in hadronic and electromagnetic configurations, at various energies of pion and electron beams. The beam test and simulation results of this prototype are reported.
\end{abstract}

Abstract Approved:

Thesis Supervisor

Title and Department

Date 
SAME SIGN DIMUON SEARCH FOR HEAVY MAJORANA MASS NEUTRINOS AT THE CMS EXPERIMENT AT CERN AND DESIGN STUDIES OF A QUARTZ PLATE CALORIMETER PROTOTYPE

\author{
by \\ Warren James Clarida
}
A thesis submitted in partial fulfillment of the requirements for the Doctor of Philosophy degree in Physics in the Graduate College of The University of Iowa

December 2012

Thesis Supervisor: Professor Yasar Onel 
Graduate College

The University of Iowa

Iowa City, Iowa

\section{CERTIFICATE OF APPROVAL}

PH.D. THESIS

This is to certify that the Ph.D. thesis of

\section{Warren James Clarida}

has been approved by the Examining Committee for the thesis requirement for the Doctor of Philosophy degree in Physics at the December 2012 graduation.

Thesis Committee:

Yasar Onel, Thesis Supervisor

Jane Nachtman

Edwin Norbeck

Mary Hall Reno

Weimin $\mathrm{Wu}$ 
To Charly, Craig, and Mike, for introducing me to a scientific view of the world. 


\section{ACKNOWLEDGMENTS}

Anyone who manages to succeed in graduate studies has an inordinate number of people to thank for their help along the way. I am not unique in this manner, and invariably by thanking a few people by name I will fail to include some who are deserving. If you happen to read this you have my apologies.

I would like to thank my advisor Prof. Yasar Onel for knowing I would find a place working in experimental physics well before I did, and supporting me through the complications of me moving from Iowa to California to Illinois and then back to Iowa again. Also, thank you to Prof. Steve Feller who got me started on this path through the excellent program he runs at Coe College.

No analysis is performed without a great deal of work from many people, in particular the Majorana search has been shepherded and pushed along tirelessly by Weimin $\mathrm{Wu}$, and now is being excellently directed by Prof. John Ellison. The Quartz Plate Calorimeter would not have continued to improve throughout these years without the constant work of Ugur Akgun as a postdoc and now professor. Another postdoc and now professor, Taylan Yetkin gave me invaluable assistance in learning how to perform physics analyses. I could never have reached this point without his guidance.

Graduate school is at times an incredibly stressful and long process. The friendships that I was lucky enough to enjoy during the process created such a warm community that often this stress was barely felt. Thank you Fermilab ultimate players, Sunday dinner crowd, lincoln karaoke, and VE climbers.

The confidence and knowledge that I needed to succeed are a direct result of the support my parents have always given me. I cannot underscore how important their belief in me has been. 
At the root of my success was the person who kept me on course every day. My girlfriend, then fiance (for far to long), and then wife Kristi would not let me lose course. Every time I felt lost she was the one who could guide me back and remind me what I was working toward. 


\begin{abstract}
This paper consists of two studies: the results of a search for heavy Majorana neutrinos $(\mathrm{N})$ using an event signature defined by two like-sign charged muons and two jets, and the results from studies of a prototype quartz plate calorimeter. The data in the Majorana search correspond to an integrated luminosity of $5.0 \mathrm{fb}^{-1}$ of pp collisions at a centre-of-mass energy of $7 \mathrm{TeV}$ collected with the CMS detector at the Large Hadron Collider. No excess of events are observed beyond the expected standard model background and therefore upper limits are set on the square of the mixing element, $\left|V_{\mu N}\right|^{2}$ as a function of Majorana neutrino mass. These are the first direct upper limits on the heavy Majorana-neutrino mixing for $m_{N}>90 \mathrm{GeV}$. The second part of this thesis is the results of performance tests of a 20-layer quartz plate calorimeter prototype. The calorimeter prototype was tested at the CERN $\mathrm{H} 2$ area in hadronic and electromagnetic configurations, at various energies of pion and electron beams. The beam test and simulation results of this prototype are reported.
\end{abstract}




\section{TABLE OF CONTENTS}

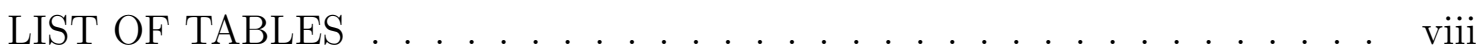

LIST OF FIGURES ........................ . . . ix

\section{CHAPTER}

1 THE STANDARD MODEL AND NEUTRINOS . . . . . . . . . . . 1

1.1 Discovery of Neutrinos . . . . . . . . . . . . . . . . . . . 1

1.2 Neutrino Mass ................... 3

2 EXPERIMENTAL APPARATUS . . . . . . . . . . . 6

$2.1 \mathrm{LHC} \ldots \ldots \ldots \ldots \ldots$

2.1.1 Proton Injection . . . . . . . . . . . . . . . . . 6

2.1.2 Beam Control . . . . . . . . . . . . . 7

2.1.3 Beam Structure . . . . . . . . . . . . . . . . . . 8

2.2 Compact Muon Solenoid Detector . . . . . . . . . . . . 8

2.2.1 Inner Tracker . . . . . . . . . . . . . . . . . . . 11

2.2.2 Electromagnetic Calorimeter . . . . . . . . . . . . 13

2.2.3 Hadronic Calorimeter . . . . . . . . . . . . . . . . . . 14

2.2.4 Muon Systems . . . . . . . . . . . . . . . . . 16

2.2.5 Trigger .................... 21

3 HEAVY MAJORANA NEUTRINO SEARCH . . . . . . . . . 24

3.1 Introduction . . . . . . . . . . . . . . . . . 24

3.2 Event Generation and Simulation . . . . . . . . . . . . . 26

3.2.1 Event Generation . . . . . . . . . . . . . . 27

3.2.2 Event Simulation . . . . . . . . . . . . . . . . 31

3.3 Muon Reconstruction . . . . . . . . . . . . . . . . . . . 32

3.4 Jet Reconstruction . . . . . . . . . . . . . . . . . . . . 33

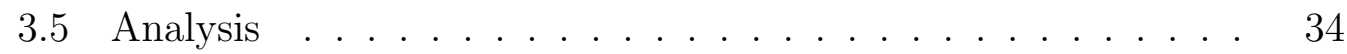

3.5.1 Data and Monte Carlo Samples . . . . . . . . . . . 34

3.5.2 Event Selection . . . . . . . . . . . . . . . . 39

3.5.3 Data-Monte Carlo Scale Factor . . . . . . . . . . . . . . 48

3.5.4 Backgrounds .................. . . 49

3.5.5 Error Analysis . . . . . . . . . . . . . . . . 56

3.5.6 Setting Exclusion Limits . . . . . . . . . . . 57

3.5.7 Results .................... 63

3.5.8 Future Prospects ............... 63

4 QUARTZ PLATE CALORIMETER PROTOTYPE . . . . . . . 66

4.1 Motivation and Introduction . . . . . . . . . . . 66 
4.2 Prototype Design _. . . . . . . . . . . . . . . 67

4.3 Prototype Testing . . . . . . . . . . . . . . . . . . 68

4.4 Summary and Future Prospects . . . . . . . . . . . . 70

BIBLIOGRAPHY . . . . . . . . . . . . . . . 77 


\section{LIST OF TABLES}

3.1 Summary of Majorana neutrino signal Monte Carlo samples generated with $\left|V_{\mu N}\right|^{2}=1$ including the calculated cross section as a function of Majorana neutrino mass. . . . . . . . . . . . . . 36

3.2 Summary of standard model Monte Carlo datasets used. . . . . . . 37

3.3 Abbreviations used in Table $3.2 \ldots \ldots$. . . . . . . . . . . . 38

3.4 Summary of 2011 datasets used. . . . . . . . . . . . . . . . . 38

3.5 Muon selection and individual cut efficiencies and statistical errors for events passing each selection criteria. . . . . . . . . . . .

3.6 Event selection efficiencies for the selection. Errors are statistical only. 43

3.7 Muon identification and isolation efficiencies for both simulation and data measured with the tag and probe method. . . . . . . . . .

3.8 Observed event yields and estimated backgrounds with statistical and systematic uncertainties are shown for the $E_{\mathrm{T}}^{\text {miss }}>50 \mathrm{GeV}$ control region. . . . . . . . . . . . . . . . .

3.9 Observed event yields and estimated backgrounds with statistical and systematic uncertainties for the analysis, with $4.98 \mathrm{fb}^{-1}$, for the signal region $\left(E_{\mathrm{T}}^{\mathrm{miss}}<50 \mathrm{GeV}\right)$. . . . . . . . . . . . .

3.10 Summary of systematic uncertainties across the mass range studied. 


\section{LIST OF FIGURES}

2.1 LHC proton injection chain at the CERN complex. . . . . . . . 9

2.2 Representation of the beam structure of the LHC, showing 25-ns intervals filled with proton bunches ("b") and gaps ("e"). . . . . .

2.3 The CMS detector, shown with sub-detector systems in the barrel and endcap regions, and forward hadron calorimeter. . . . . . . .

2.4 Inner Tracker of CMS, consisting of a pixel detector closest to the interaction point, followed by layers of silicon strip detctors in the inner barrel (TIB), outer barrel (TOB), inner disc (TID), and endcap (TEC) regions. . . . . . . . . . . . . . . . .

2.5 CMS Electromagnetic Calorimeter: Cross section of modules in the barrel region and endcap, "Dee", components in the endcap region.

2.6 CMS Hadronic Calorimeter: barrel (HB), endcap (HE), outer (HO), and forward $(\mathrm{HF})$ regions. . . . . . . . . . . . . .

2.7 Cross sectional view of a drift tube used in the CMS muon subdetector.

2.8 Location of the drift tube modules in the CMS barrel region. . . . .

2.9 Location of cathode strip chamber modules in CMS endcap region.

2.10 Cross sectional view of a cathode strip chamber. . . . . . . . . . . 20

2.11 Location of resistive plate chamber in CMS barrel region. . . . . . . 21

2.12 Diagram of information flow in the Level 1 trigger chain in CMS. .

3.1 The Feynman diagram for resonance production of a Majorana neutrino $(N)$. The charge-conjugate diagram results in a $\ell^{-} \ell^{-} q \bar{q}^{\prime}$ final state.

3.2 The cross section for resonance production of a Majorana neutrino as a function of Majorana neutrino mass from two independent event generators. . . . . . . . . . . . . . . .

3.3 Parton Distribution Functions from the CTEQ collaboration for two values of interaction scale $\mathrm{Q} . \ldots \ldots \ldots$

3.4 Typical event display of a dimuon event passing this analysis' selection cuts. The $\rho-\phi$ plane is shown. . . . . . . . . . . . 
3.5 Typical event display of a dimuon event passing this analysis' selection cuts. The $\rho-z$ plane is shown. . . . . . . . . . . . . .

3.6 Typical event display of a dimuon event passing this analysis' selection cuts. A 3D image of the event is shown. . . . . . . . . . .

3.7 Typical event display of a dimuon event passing this analysis' selection cuts. The calorimeter energy deposits in an $\eta-\phi$ graph is shown. . . . . . . . . . . . . . . . . .

3.8 This shows an example fit to a dimuon mass spectrum from the $\mathrm{Z}+$ jets Monte Carlo listed above where probe muon was required to pass the full isolation cuts and have a $p_{\mathrm{T}}$ above $20 \mathrm{GeV}$. . . . .

3.9 This shows an example fit to a dimuon mass spectrum from the data using the fit from Figure 3.8; where again the probe muon was required to pass the full isolation cuts and have a $p_{\mathrm{T}}$ above $20 \mathrm{GeV}$. .

3.10 Observed exclusion region in the Majorana neutrino mixing element squared vs. mass plane obtained using the $C L_{s}$ method is above the red line; where the black line is the expected limit (with one and two sigma bands in green and yellow respectively) and also shown are the limits from DELPHI and L3. . . . . . . . . . . . . . . . .

3.11 Comparison of the exclusion region in the Majorana neutrino mixing element squared vs. mass plane from the 2011 data above the red line study and the possible exclusion from the 2012 data above the black line. . . . . . . . . . . . . . . . 65

4.1 Different fiber geometries on plates. . . . . . . . . . . . . . . 69

4.2 This is an image of four of the layers created in the GEANT4 Simulation 71

4.3 The $300 \mathrm{GeV}$ pion response of the calorimeter prototype in the hadronic configuration; where one QIE count corresponds to $2.7 \mathrm{fC}$. . . . . .

4.4 The $100 \mathrm{GeV}$ electron response of the calorimeter prototype in the EM configuration; where one QIE count corresponds to 2.7 fC. . . .

4.5 The hadronic resolution and statistical error of the quartz plate calorimeter prototype is shown for data (solid red line - circles) and GEANT4 simulations (black dashed line-squares). . . . . . . . . . . 74

4.6 The hadronic linearity and statistical error of the quartz plate calorimeter prototype with a coefficient of determination, $R^{2}$, from the fit of

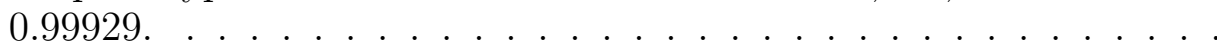


4.7 The electromagnetic resolution and statistical error of the quartz plate calorimeter prototype is shown for data (solid red line - circles) and GEANT4 simulations (black dashed line - squares). . . . .

4.8 The electromagnetic linearity and statistical error of the quartz plate calorimeter prototype; in this case with an $R^{2}$ from the fit of 0.99999 . 76 


\section{CHAPTER 1 THE STANDARD MODEL AND NEUTRINOS}

The fundamental concern of physics is understanding the structure of the universe. This understanding underwent a revolution in the early 20th century when a series of discoveries concerning the underlying nature of matter and the dynamics of interactions. These discoveries led to the advent of modern or quantum physics. Over the last century the foundations of our universe and the questions that arose from those first discoveries have been explore in exhaustive detail. This led to the development of the Standard Model (SM) a theoretical framework that describes all the known constituents of our universe and nearly all the dynamics. The history and current formulation of the standard model has been discussed many times; a thorough overview can be found in David Griffiths' particle physics textbook [1].

While the the SM has been incredibly successful it is not a complete description of the makeup and dynamics of our universe. There are several open questions still to be answered; one example is the recent discovery that neutrinos are not massless, but instead have a rest mass that when compared to the other SM particles is surprisingly small $[2,3,4,5]$. The SM as currently constructed does not predict massive neutrinos. A simple extension of the SM however would allow for the small neutrino masses. The simplest solutions calls for the addition of new heavy neutrinos $[6,7,8,9]$. The search for the signature of one type of such a new heavy neutrino is presented in this thesis.

\subsection{Discovery of Neutrinos}

In the early 20th century the study of beta decay, conversion of a neutron into a proton, led to a potential problem with conservation of energy. The visible decay products of beta decay, an electron and a proton were found to have a continuous 
energy spectrum in the rest frame of the neutron, whereas in true two body decay, the two decay products should be produced back to back with equal momenta. The addition of a third decay product would fix this problem, and that is what Pauli proposed in 1930 [10], to be later formally treated by Fermi in 1934 [11].

Bubble chamber experiments over the next two decades provided ample theoretical evidence for the existence of the neutrino. When a pion or muon decays in a bubble chamber the charged track of its decay product can be seen and the decay vertex is observed as a kink in the charged tracks. This kink offers increased evidence of the neutrino. As with beta decay, without an additional neutral particle being added to the decay, energy cannot be conserved.

This was not sufficient evidence for the neutrino, however, just that it made a convenient book-keeping method for maintaining energy conservation. The actual discovery of the neutrino required the observation of reverse beta decay.

$$
\nu+p \rightarrow n+e^{+}
$$

The incredibly small cross-section of this process made the discovery of the neutrino very difficult even with the large neutrino flux provided by uranium reactors. The discovery was finally made by Reines and Cowan in 1956 using the neutrino flux from the Savannah River nuclear power plant in South Carolina. They built two large detectors comprised of $C d C l_{2}$ dissolved in water. This allowed them to observe the gamma rays from the decay of the positron and an associated gamma ray produced by the neutron being absorbed by $\mathrm{Cd}$ nuclei. Their result was found after taking several months of data at a collection rate of a few events per hour. They measured a cross-section of $6.3 \times 10^{-44} \mathrm{~cm}^{2}$ [12] 


\subsection{Neutrino Mass}

The standard model as presently constructed does not require massive neutrinos. The neutrinos present in the standard model interact only via the weak force, referred to as interacting weakly. After electroweak symmetry is broken in the Standard Model the charged current interactions in the Lagrangian have as part of their coupling the chiral projection operator, this means that the coupling to the $\mathrm{W}$ bosons is done only by left-handed particles (or right-handed anti-particles). Thus neutrinos have only been detected in left-handed states (or right-handed in the case of anti-neutrinos). The normal way of inserting a mass term for a massive particle into the standard model, called a Dirac mass, requires both right and left handed states. So long as neutrinos are massless this, presents no problem to the Standard Model's construction (alternatively, the right handed state could be a "sterile" neutrino having almost no interaction with other known particles). As there was no theoretical need for neutrinos to have mass, it was thought for a long time that they were indeed massless. An alternative type of mass called a Majorana mass is described in Chapter 3.

Beginning in the late 1960's a series of experiments noticed similar discrepancies: that the solar neutrino flux seemed to be lower than could be expected from current models of the Sun. This was first noticed with a detector in the Homestake mine $[13,14]$ in South Dakota, but was quickly replicated by many other solar neutrino observational experiments. The Particle Data Group provides a comprehensive list [15]. For many years following, attempts were made to understand how models of the Sun could be corrected to solve this problem (a review of these proposals can be found in Reference [16]). The alternative explanation that neutrinos had mass had been known for almost as long as the problem had been present [17], but it was generally believed that neutrinos were massless. 
The first clear evidence for neutrino mass as opposed to errors in solar models being the source of the deficit came with a measurement of the solar neutrino flux after passing through the Earth (i.e., the flux at night) versus the flux during the day [18]. Neutrinos interact at a sufficiently low rate that the solar neutrino flux is not impacted significantly by the earth so this flux should be the same. If the flux is not the same it is evidence that the solar neutrinos are oscillating into different neutrino flavors which in turn implies that neutrinos have mass. Neutrino oscillation is evidence of neutrino mass because, in general the flavor states of neutrinos that are observed in detectors can be superpositions of the mass states. Over a sufficient distance a neutrino existing in a specific mass state may oscillate into another flavor as a neutrino with single mass state can be a superposition of flavor states.This concept of neutrino mixing was first studied by Pontecorvo, Maki, Nakagawa [19], and Sakata [20] leading to the neutrino mixing matrix sometimes being called the PMNS matrix. So if neutrinos did oscillate then the solar neutrino flux would be different during the day than during the night due to the extra distance traveled through the Earth, and this is indeed what was observed. Following this the Sudbury Neutrino Observatory measured not only the electron neutrino deficit but also the increase in the muon and tau neutrino flux [21]. The deficit in electron neutrinos matched the increased flux of the other flavor neutrinos. This was finally definitive evidence of neutrino oscillation, in turn, requiring that neutrinos be massive particles.

Once it was established that neutrinos are massive particles, the methods for including their mass into the standard model moved from a niche area of particle physics to a mainstream search. In their preface to the book Massive Neutrinos in Physics and Astrophysics (3rd Edition) [2] the authors state:

...neutrino mass has moved from being a matter of faith to a stark reality... The first definitive sign of physics beyond the standard model has 
finally appeared. This is a revolution that calls for a celebration.

This book [20] provides an extensive review of the formalism of the various models that provide methods for inserting massive neutrinos into the Standard Model. The most popular model, which serves as the basis for the analysis presented later in this document, is termed the "see-saw" mechanism. This mechanism accounts for the small size of the observed neutrino masses with the introduction of a new heavy state $N$. This results in light neutrino mass terms with the structure: $m_{\nu} \sim y_{\nu}^{2} v^{2} / M_{N}$, where $y_{\nu}$ is a Yukawa coupling and $v$ is the Higgs vacuum expectation value in the Standard Model. A search for particles which could fulfill the role of the new heavy mass state is the focus of the analysis presented in Chapter 3. 
CHAPTER 2

EXPERIMENTAL APPARATUS

\section{$2.1 \mathrm{LHC}$}

While there are many means by which the basic structure of the universe can be studied, the primary method used to establish the standard model was accelerator based experiments starting with fixed target experiments and then switching to colliders about 50 years ago. The use of the experiments is driven by the need to study particles that cannot be reliably found in a controlled way in nature. Most of the particles being studied here are massive particles produced by the collision of two particles, either from one accelerated particle beam hitting a fixed target or with modern colliders the collision of two accelerated beams. These massive particles then decay into the final state products that are detected in modern detectors. The Large Hadron Collider (LHC) at the European Organization for Nuclear Research (CERN) is currently the state of the art collider in the world. It accelerates protons to nearly the speed of light in two counter rotating beams, and then focuses the beams into crossing points at four locations around the accelerator ring. The full accelerator complex is shown in Figure 2.1 [22].

\subsubsection{Proton Injection}

The protons used in the accelerated particle beams are sourced from hydrogen gas. The hydrogen molecules are ionized in a duoplasmatron operating at $100 \mathrm{kV}$. The ionized gas is then propelled into a cathode chamber and dissociates, providing the protons needed by the CERN accelerators.

The protons then proceed through a number of steps through multiple accelerators which slowly bring up their energy. From the cathode chamber the protons 
are injected in to the Linac 2 (linear accelerator 2). This process performed by magnetic fields first creates a beam structure as the protons are forced through a canal for injection. The Linac 2 brings the beam energy up to $50 \mathrm{MeV}$ which is about $30 \%$ of the speed of light. From there the beam moves into the Proton Synchrotron Booster (PSB) where it is accelerated to $1.4 \mathrm{GeV}$. The Proton Synchrotron (PS) then gives the beam an additional energy up to $28 \mathrm{GeV}$. At this point the PS also arranges the beam of protons into bunches with a nominal spacing of $25 \mathrm{~ns}$. The bunched beam is fed into the Super Proton Source (SPS) that increases the beam energy up to $450 \mathrm{GeV}$. At this point many different experiments are fed by the SPS.

In order to build large experiments like those at the LHC extensive detector design and testing must be done. One of the uses for the $450 \mathrm{GeV}$ SPS beam is a test beam facility where detector prototypes can be placed in beams of known energy and composition allowing for the detector to be characterized. Additionally, the SPS protons can be used for non-collision experiments focused on studying specific aspects of the standard model; one such experiment is the production of neutrino beams for the accelerator-based neutrino physics experiments mentioned above. Finally, the majority of the protons from the SPS are injected in the LHC ring. At this point the beams are accelerated to the full collision energy of $3.5 \mathrm{TeV}$ [23] for the 2011 run and $4 \mathrm{TeV}$ for the 2012 run. The full design energy of the LHC is $7 \mathrm{TeV}$ per beam.

\subsubsection{Beam Control}

The LHC ring is composed of $27 \mathrm{~km}$ of dipole and quadropole cryogenically cooled magnets in a tunnel lying 50 to $100 \mathrm{~m}$ underneath the French Swiss border outside the Swiss city of Geneva. The protons are accelerated by RF cavities operating at $400 \mathrm{MHz}$ while the 1232 dipole magnets steer the beam around the ring and the 386 quadropoles focus the beams to a diameter of about $20 \mu \mathrm{m}$ for collisions 
at each of the four crossing points. As charged particles radiate when accelerated (i.e., changed direction around the ring) the RF cavities must constantly maintain the beam energy. Their energy is provided by 16 klystrons which each provide an output of $300 \mathrm{~kW}$ for a total power output of $4800 \mathrm{~kW}$ [23].

\subsubsection{Beam Structure}

As mentioned above the beam has a bunch structure, where there are, for the most part 25 ns gaps between proton bunches. Not every one of the 3564 bunch buckets is filled however, as shown in Figure 2.2; this provides opportunities for the beam to be steered in a different direction. Specifically it can be steered into a beam dump under the Jura Mountains. If this gap structure were not present the beam would sweep out through sensitive instruments during occasions where it needed to be discarded. Such an event could cause extensive damage and irradiate areas of the tunnel complicating repair. The beam dump gap is placed between the bunches numbered 3445 and 3564. Also shown in the figure are other smaller gaps present from the beam dump gaps in the previous accelerators discussed above.

\subsection{Compact Muon Solenoid Detector}

The Compact Muon Solenoid (CMS) is one of four detectors at the LHC. It is one of two general purpose detectors built primarily for the discovery of new physics. The full specification of the detector is described in Reference [24]. This document provides a brief description of the various components. Detector schematics are also taken from Reference [24].

CMS has four major subdetector units each of which are layered into a cylindrical barrel with two end caps; this is depicted in Figure 2.3 [25]. The inner three subdetectors in the barrel are placed inside the $3.8 \mathrm{~T}$ solenoid for which the detector is partially named. Working outward from the collision point at the center of the 


\section{CERN's accelerator complex}

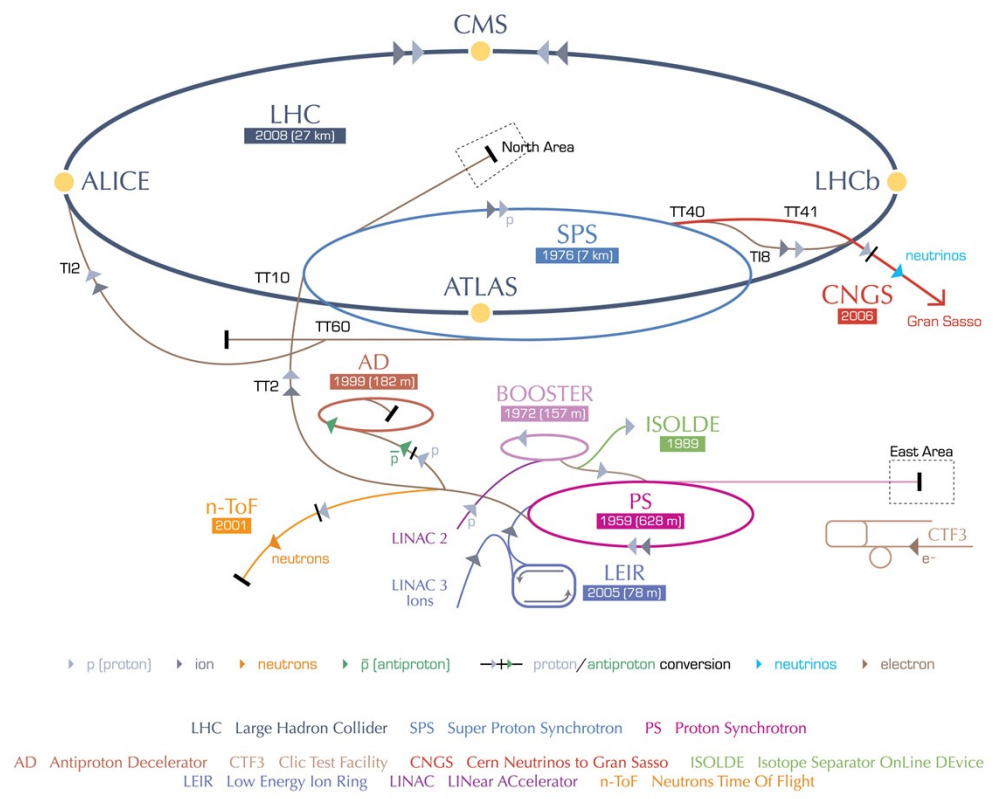

European Organization for Nuclear Research | Organisation européenne pour la recherche nucléaire

O CERN 2008

Figure 2.1: LHC proton injection chain at the CERN complex.

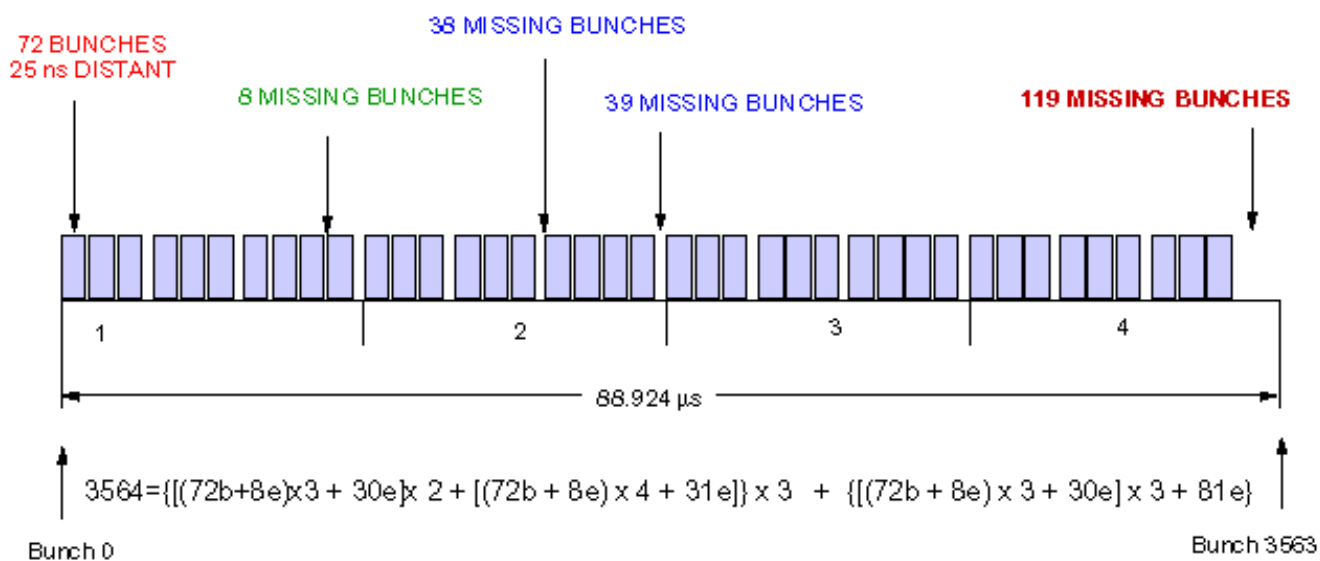

Figure 2.2: Representation of the beam structure of the LHC, showing 25-ns intervals filled with proton bunches ("b") and gaps ("e"). 
detector the various subdetectors are:

- Tracker Measures with high precision the paths of charged particles.

- Electromagnetic Calorimeter Measures the energy of electrons and photons and also contributes to the measurement of the energy coming from hadronic showers.

- Hadronic Calorimeter Measures the energy of hadronic showers.

- Solenoid Provides magnetic field causing charged particles to have a curved trajectory.

- Muon Tracker Measures the path of a muon leaving the detector.

In addition to the subdetectors there is an online trigger system which chooses the events to be saved based upon information provided from each subdetector. The trigger is discussed further in Section 2.2.5.

CMS uses a cylindrical coordinate system defined with the $\mathrm{z}$ axis aligned along the beam axis, the azimuthal angle $\phi$ is measured from $-\pi$ to $\pi$ where $\phi=0$ is defined as point inward along the LHC ring radius, and finally the angle $\theta$ measured from the $\mathrm{z}$ axis runs from 0 to $\pi$. The angle $\theta$ is often replaced by the pseudorapidity, $\eta$ defined:

$$
\eta=-\ln \left(\tan \left(\frac{\theta}{2}\right)\right)=\frac{1}{2} \ln \left(\frac{|p|+p_{L}}{|p|-p_{L}}\right)
$$

Here, $p_{L}$ is the $\mathrm{z}$ component of the particle's momentum, and $|p|$ is the magnitude of the particles full momentum. The coordinate $\eta$ is a convenient coordinate because in minimum bias events particle production is constant as a function of $\eta$ at the zero mass limit. Minimum bias events are bunch crossings where at least one interaction is required in the bunch crossing. Additionally, the angle between two particles 
when measured with $\eta$ is invariant under Lorentz boosts.

\subsubsection{Inner Tracker}

The innermost subdetector, the tracker, is composed of two parts a high resolution pixel detector closest to the interaction point followed by a silicon strip tracker. The pixel detector starts at a radial distance of $4.4 \mathrm{~cm}$ from the crossing point and continues out to a radius of $10.2 \mathrm{~cm}$. The silicon strip detector then continues out to $1.1 \mathrm{~m}$ as shown in Figure 2.4.

Each bunch crossing will have an average of 1000 particles hitting the inner face of the pixel detector. This combined with the 25ns bunch spacing leads to a hit rate density of $1 \mathrm{MHz} / \mathrm{mm}^{2}$. The CMS detector's physics requirements lead to a need for a single channel occupancy of $1-3 \%$. These specifications define the requirements of the pixel detector and its designed coverage out to $10 \mathrm{~cm}$.

Each pixel is composed of a thin layer of depleted silicon, so that when a charged particle passes through it create electron hole pairs. These are separated by a $300 \mathrm{~V}$ bias voltage and the resulting charge buildup is read out by an active amplifier. In the barrel region there are 3 layers of pixels and then two layers in each endcap disc. This gives a total of 1440 modules and 66 million pixels.

Beyond $10 \mathrm{~cm}$ the particle flux is reduced enough (because some particles are stopped by the pixel detector) that silicon strip detectors can be used instead of pixels. Silicon strips give two dimensional position information from successive perpendicular strips as opposed to the three dimensional information provided by a single pixel. Each silicon strip is a six inch wafer of n-doped silicon with $p^{+}$implants on the front side and $n^{+}$on the back side. This is done to increase the lifetime of the detector; once radiation damage leads to inversion of the silicone to p-type it will still be able to use the $\mathrm{p}$ - $\mathrm{n}$ junction. The barrel region has 10 layers of strips while the endcap has 12 . In the endcap the inner 3 layers are discs with a radius of 


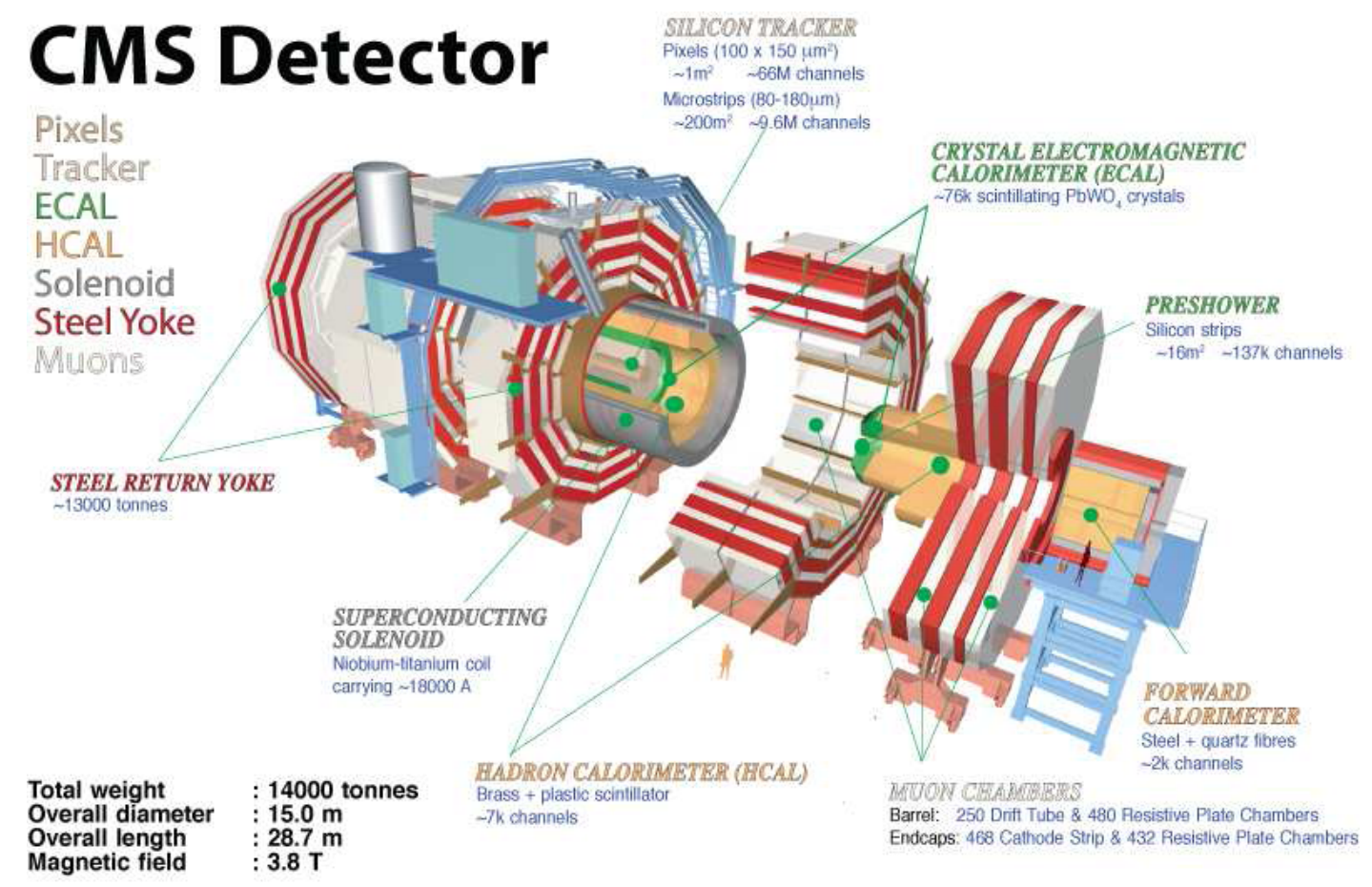

Figure 2.3: The CMS detector, shown with sub-detector systems in the barrel and endcap regions, and forward hadron calorimeter.

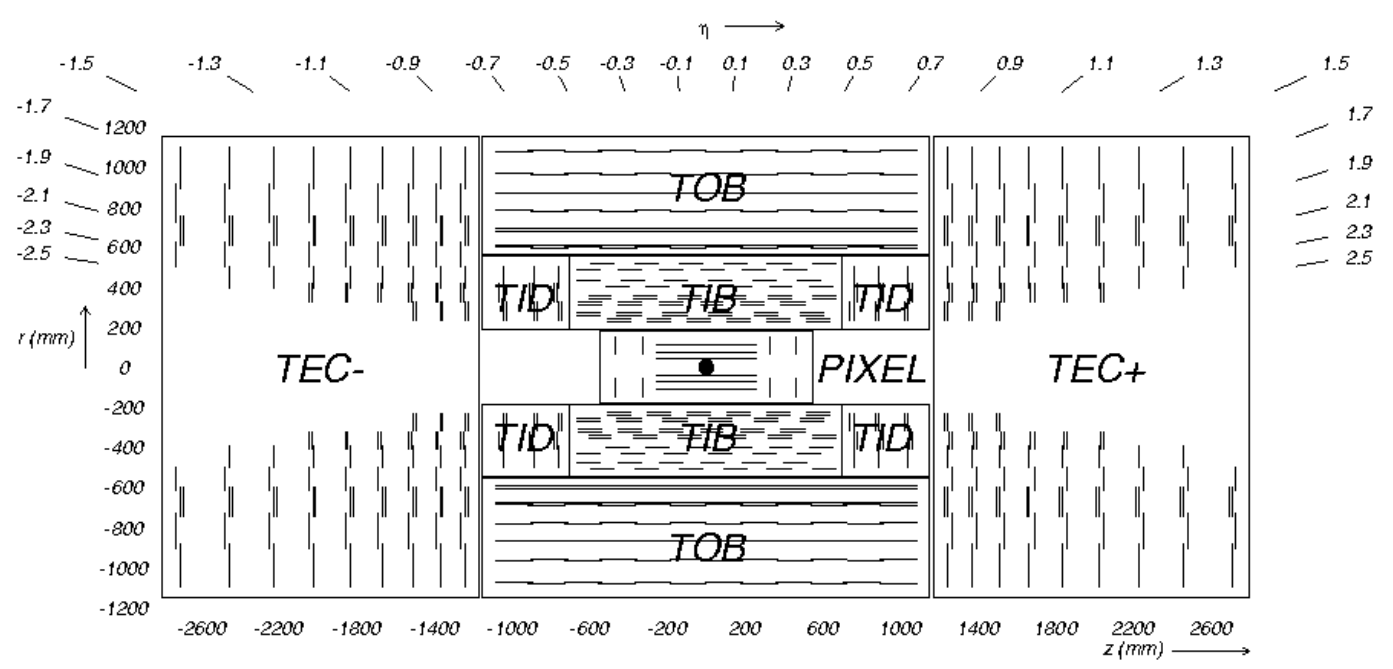

Figure 2.4: Inner Tracker of CMS, consisting of a pixel detector closest to the interaction point, followed by layers of silicon strip detctors in the inner barrel (TIB), outer barrel (TOB), inner disc (TID), and endcap (TEC) regions. 
$55 \mathrm{~cm}$ while the outer 9 layers cover out to $113.5 \mathrm{~cm}$. There are a total of 93 million strips.

\subsubsection{Electromagnetic Calorimeter}

The electromagnetic calorimeter (ECAL) is a homogeneous calorimeter, that is a calorimeter in which the absorbing material and the sensitive material that produces a signal are one and the same. The ECAL is made up of completely transparent lead tungstate $\left(\mathrm{PbWO}_{4}\right)$ crystals. they are cut in a truncated pyramidal shape so as to be essentially projective toward the primary vertex. This minimizes the amount of material along particle trajectories in which decays showers are not recorded. This can be seen in the ECAL schematic shown in Figure 2.5. The design of ECAL had to consider several constraints, the major one being the 25ns bunch crossing rate. $\mathrm{PbWO}_{4}$ provides an excellent material for dealing with this engineering challenge because $80 \%$ of the scintillation light is produced within 25ns. Also with a Moliere radius of $2.2 \mathrm{~cm}$ and a radiation length of $0.89 \mathrm{~cm}$ the electromagnetic showers in $\mathrm{PbWO}_{4}$ are short enough to allow for the homogeneous construction of the ECAL. The ECAL has a total of 75,688 crystals: 61,200 in the barrel region (EB) and an additional 7,244 in each endcap (EE).

As charged particles pass through the crystals they scintillate producing light in the 420 - $430 \mathrm{~nm}$ range. This light reflects off the polished sides of the crystals to an electronic readout. The readout is done using avalanched photodiodes (APDs) in the barrel and vacuum phototriodes (VPTs) in the endcap. The choice of VPTs in the endcap is made because of the higher radiation environment and the nonuniformity of the magnetic field from the solenoid. VPTs have only a single gain state an are therefore less affected by these complications. APDs and VPTs produce charge proportional to the amount of light reaching them. The readout is therefore accomplished by measuring the amount of charge produced by the APDs or VPTs 
which is proportional to the energy of the originating particle. APDs and VPTs have less than 2 ns rise times, so when combined with the fast response of the crystal's scintillation almost all the light can be collected in one $25 \mathrm{~ns}$ time slice.

While the crystals are initially transparent radiation damage will form color centers over time. The amount of light reaching the APDs and VPTs therefore will not be constant for a given energy. This transmission loss is calibrated for with a laser light shown into ECAL during the beam gaps discussed above.

\subsubsection{Hadronic Calorimeter}

As with ECAL the hadronic calorimeter (HCAL) has barrel and endcap components, but it also has an additional two components: an outer detector (HO) outside the solenoid and a forward detector (HF) beyond the endcap muon system

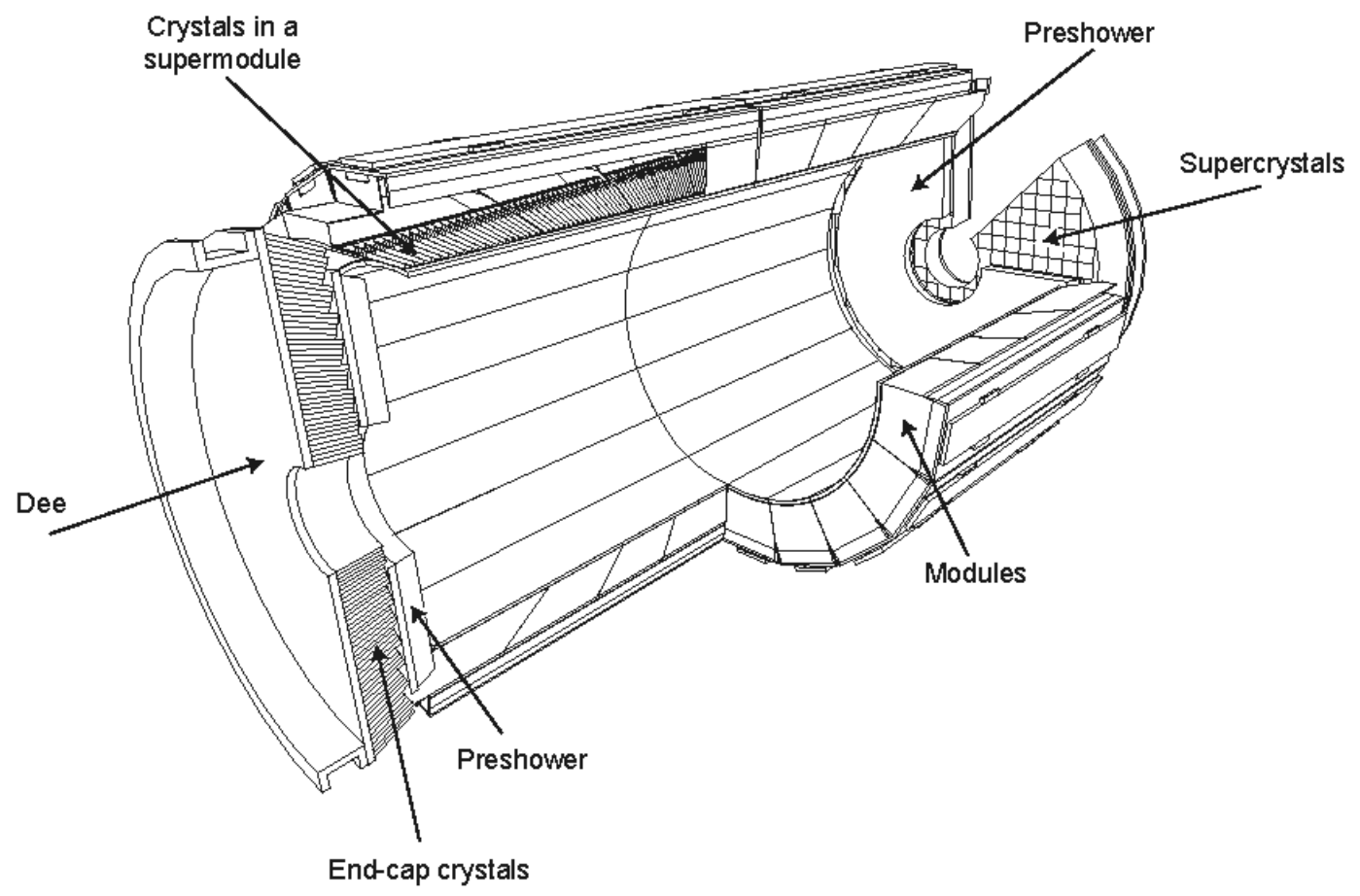

Figure 2.5: CMS Electromagnetic Calorimeter: Cross section of modules in the barrel region and endcap, "Dee", components in the endcap region. 
$11.2 \mathrm{~m}$ from the primary vertex. The four components can all be seen in Figure 2.6. HCAL is also unlike ECAL in that it is a sampling calorimeter using separate alternating absorber and active scintillating materials. The scintillating material is composed of Kuraray SCSN81 tiles while the absorber is either steel or brass. In the barrel region (HB) the absorber starts with a $40 \mathrm{~mm}$ front steel plate. Following this there are 14 brass plates with varying thicknesses (50.5 to $56.5 \mathrm{~mm}$ ) placed between the tiles. Finally there is a $75 \mathrm{~mm}$ thick steel back plate. The tiles between each metal plate are $3.7 \mathrm{~mm}$ thick. In most of the endcap (HE) there are 19 layers of brass plates with $9 \mathrm{~mm}$ gaps for the tiles. In the area where HE and HB overlap there are as few as six HE layers. This overlap minimizes the amount of dead material where particles can pass without being detected.

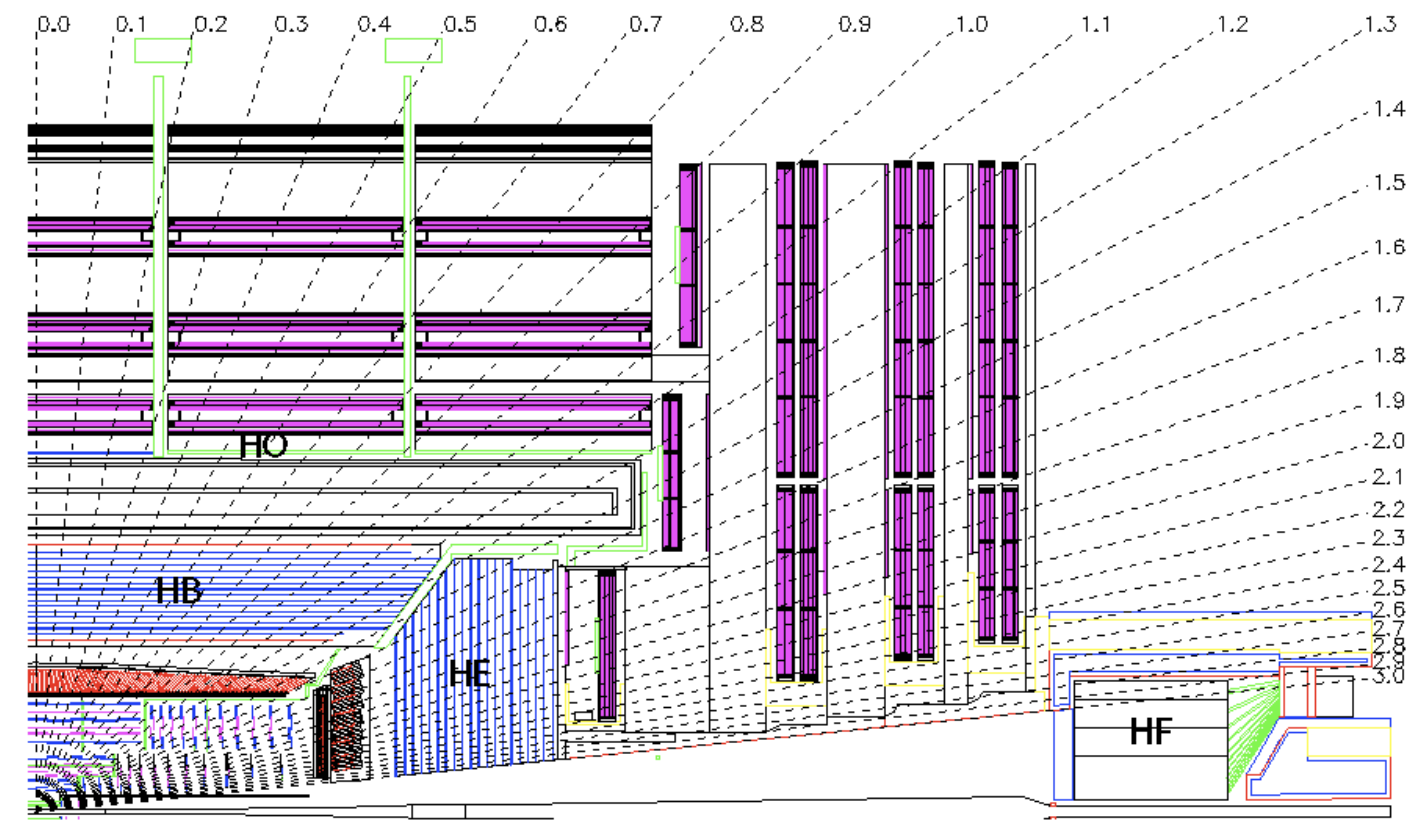

Figure 2.6: CMS Hadronic Calorimeter: barrel (HB), endcap (HE), outer (HO), and forward (HF) regions.

As hadronic particles pass through the absorber they shower. The particles then scintillate in the tiles. The scintillation light is collected by Kuraray Y-11 
double clad wavelength shifting (WLS) fibers. The fibers transport the light to high-voltage hybrid photo diodes (HPDs). The overall energy of the shower as measured by the light being conveyed to the HPDs is a sample of the original particles energy.

Showers from the most energetic particles could pass fully through HB. Called "punch through" HO is intended to detect these particles. HO is an additional layer of scintillating material outside the solenoid. The first layer of the steel magnetic field return yoke for the solenoid serves as the absorber. In the most central region there are two layers of scintillator on either side of the absorber. The rest of the barrel region has one layer on the outside of the absorber.

The forward calorimeter (HF), which was designed by the University of Iowa, serves to detect forward jets; that is, hadronic showers at high pseudorapidity. HF overlaps with $\mathrm{HE}$ and then extends to an $|\eta|$ of 5.2. This detector is located in an incredibly high radiation environment both from collision particles and from beam halo particles which led to unique design challenges. Scintillation tiles would not work under such high radiation. Instead quartz fibers are used as the active material. Steel serves as the absorber. The fibers lie in machined grooves parallel to the $\mathrm{z}$ axis. When a charged particle passes through the fibers they create Čerenkov radiation. The fibers then direct the light to photomutiplier tubes (PMTs) at the back of the subdetector. As there is no ECAL component in front of HF two different fiber lengths are used. Longer fibers detect the shorter (in depth) electromagentic showers, while shorter fibers only see particles from hadronic showers.

\subsubsection{Muon Systems}

All of the proceeding subdetector systems are enclosed within the CMS solenoid. Surrounding the solenoid is a large steel superstructure providing the structural stability for the detector. The steel yokes also serve as the return for the magnetic field 
lines of the solenoid helping the field uniformity within the solenoid. Between these layers of steel are placed the muon tracking layers. There are three different type of muon systems: drift tubes (DTs), cathode strip chambers (CSCs) and resistive plate chambers (RPCs).

The CMS DTs are rectangular tubes two to three meters long filled with an $\mathrm{Ar} / \mathrm{CO}_{2}$ gas mixture. A schematic is shown in Figure 2.7 [26]. Running down the center of each tube is an anode wire maintained at $3.7 \mathrm{kV}$ voltage. When a charged particle passes through the gas, the gas is ionized. The charge is then attracted to the anode wire; the resulting charge separation between the cathode and the anode creates a potential difference that can be measured. The time between the bunch crossing (from the CMS global clock) and the current registering give the particle's distance from the wire. The electrode strips on the top and bottom of the DT combined with the cathode strips on the side help maintain a uniform drift velocity through the DT. This affect is shown in Figure 2.7 where the contour lines are isochrones, lines along which the drift time will be the same. Multiple signals from different DT can be combined together to obtain a three dimensional track. The DT are used only in the barrel region of CMS. As can be seen in Figure 2.8 there are four layers of DTs. Each layer has three sets of tubes, two parallel to the beam and one perpendicular to the beam. The full DT system has about 172,000 wires.

Shown in Figure 2.9, the CMS endcap region uses CSCs rather than DTs. As mentioned before the endcap region has significantly more radiation and a less uniform magnetic field, and as with HE this is the reason for choosing a different detector system. CSCs are trapezoidal panels separated with $\mathrm{Ar} / \mathrm{CO}_{2} / \mathrm{CF}_{4}$ gas mixtures. Also in the intervening space are planes of anode wires while the panels are lined with cathode strips. The cathode strips follow radial lines outward from 


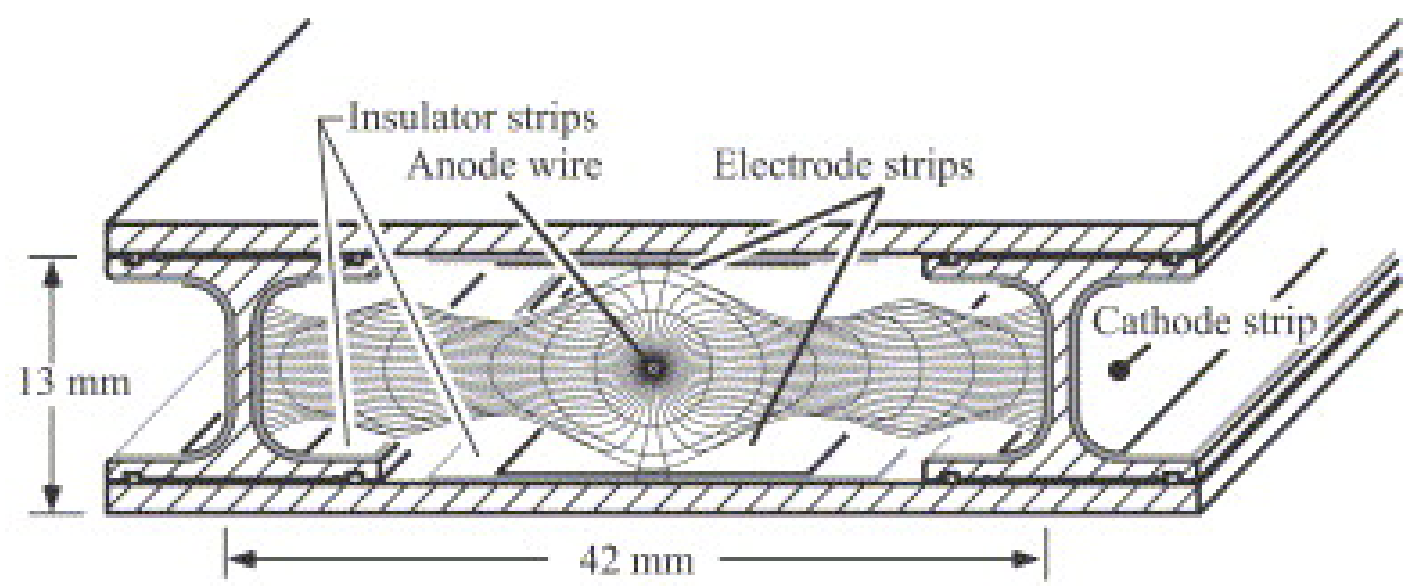

Figure 2.7: Cross sectional view of a drift tube used in the CMS muon sub-detector.

the $\mathrm{z}$ axis and also run perpendicular to the anode wires. A cross sectional view of this layout is shown in Figure 2.10

The detection mechanism is very similar to the DTs, when a charged particle passes through the gas mixture ionized gas molecules are once again created. Again the charges separate and there is a charge created on the anode, and an associated image charge on the cathode. Both currents are read out which gives a two dimensional path of the muons track. There are about 220,000 cathode strips and 180,000 anode wires in the CSC system.

An additional detector system is included in the muon subdector for muon specific triggering. RPCs provide a fast trigger system for the easily detected (compared to other types of particles) muons. RPCs consist of parallel plate double-gap gaseous chambers in both the barrel and endcap regions. A high voltage readout strip is located in the chamber's center separating the two gaps. The ionized gas in both gaps is summed together to produce a signal. The small width of each gap creates faster response and therefore faster timing from the RPCs. The charge integration is less than the $25 \mathrm{~ns}$ bunch crossing time, so that a signal can be uniquely associated with one bunch crossing. 


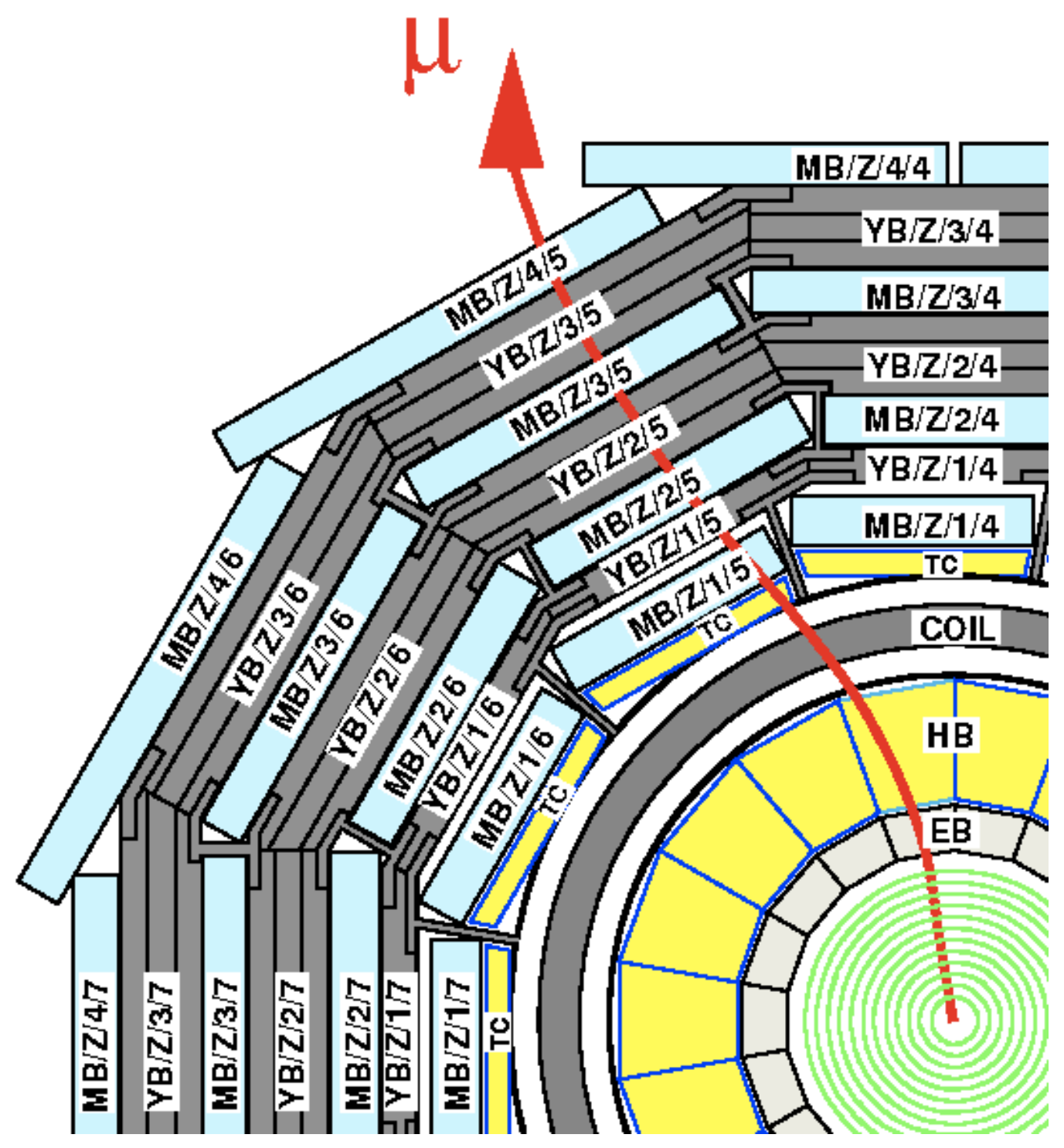

Figure 2.8: Location of the drift tube modules in the CMS barrel region. 


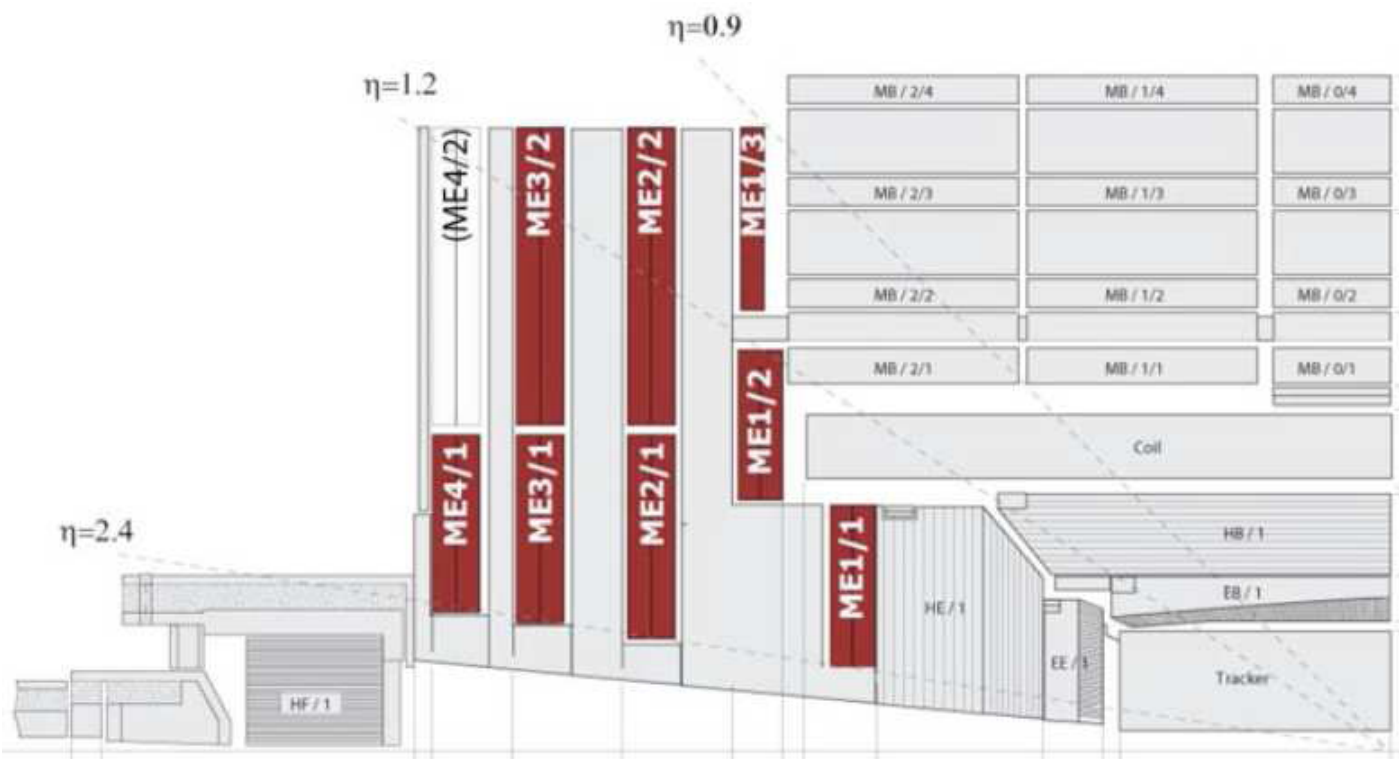

Figure 2.9: Location of cathode strip chamber modules in CMS endcap region.

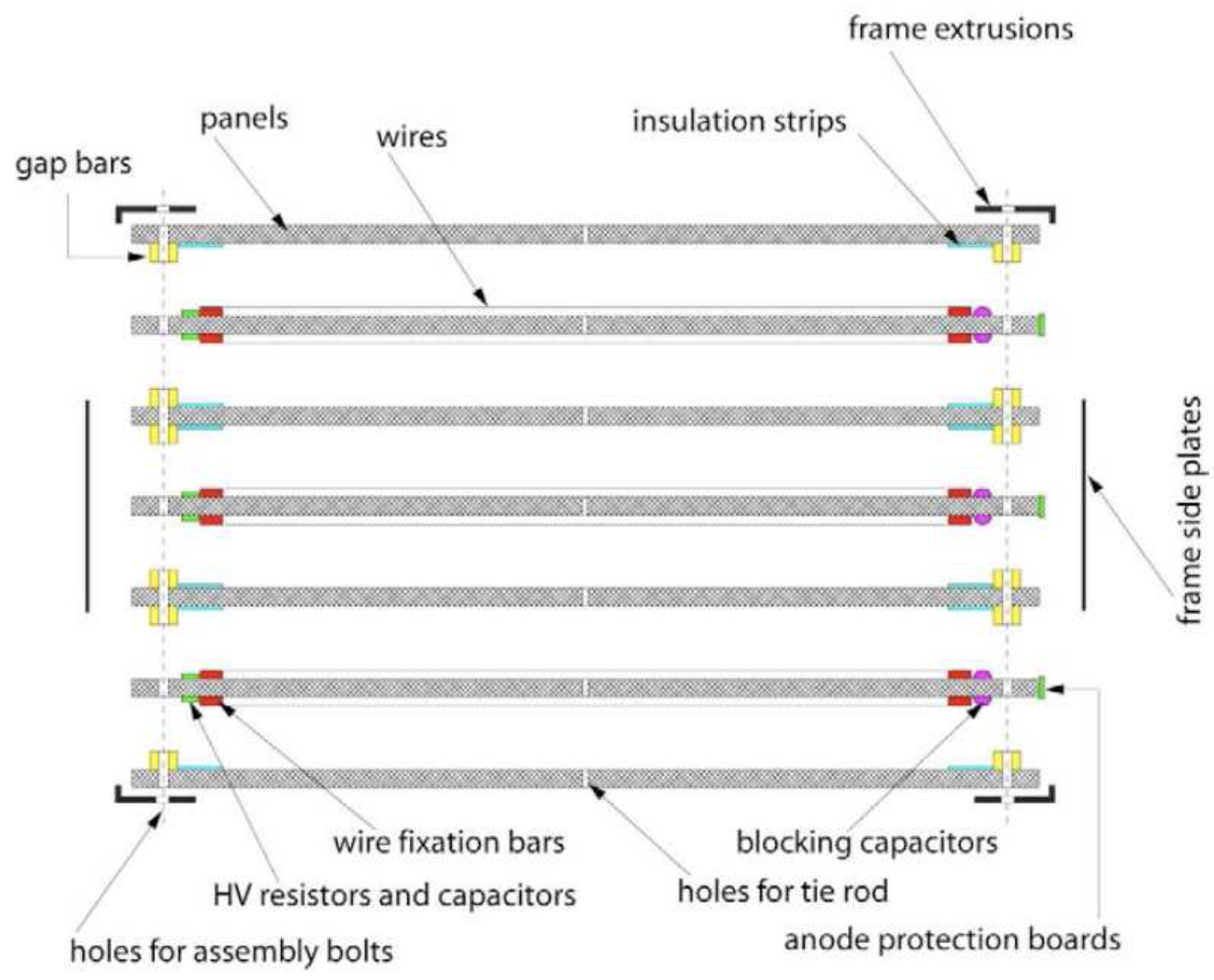

Figure 2.10: Cross sectional view of a cathode strip chamber. 
In the barrel there is one RPC on either side of a DT for the first two layers and an additional RPC on the inside of the final two layers. In the endcap there are three RPC layers. The full layout can be seen in Figure 2.11.

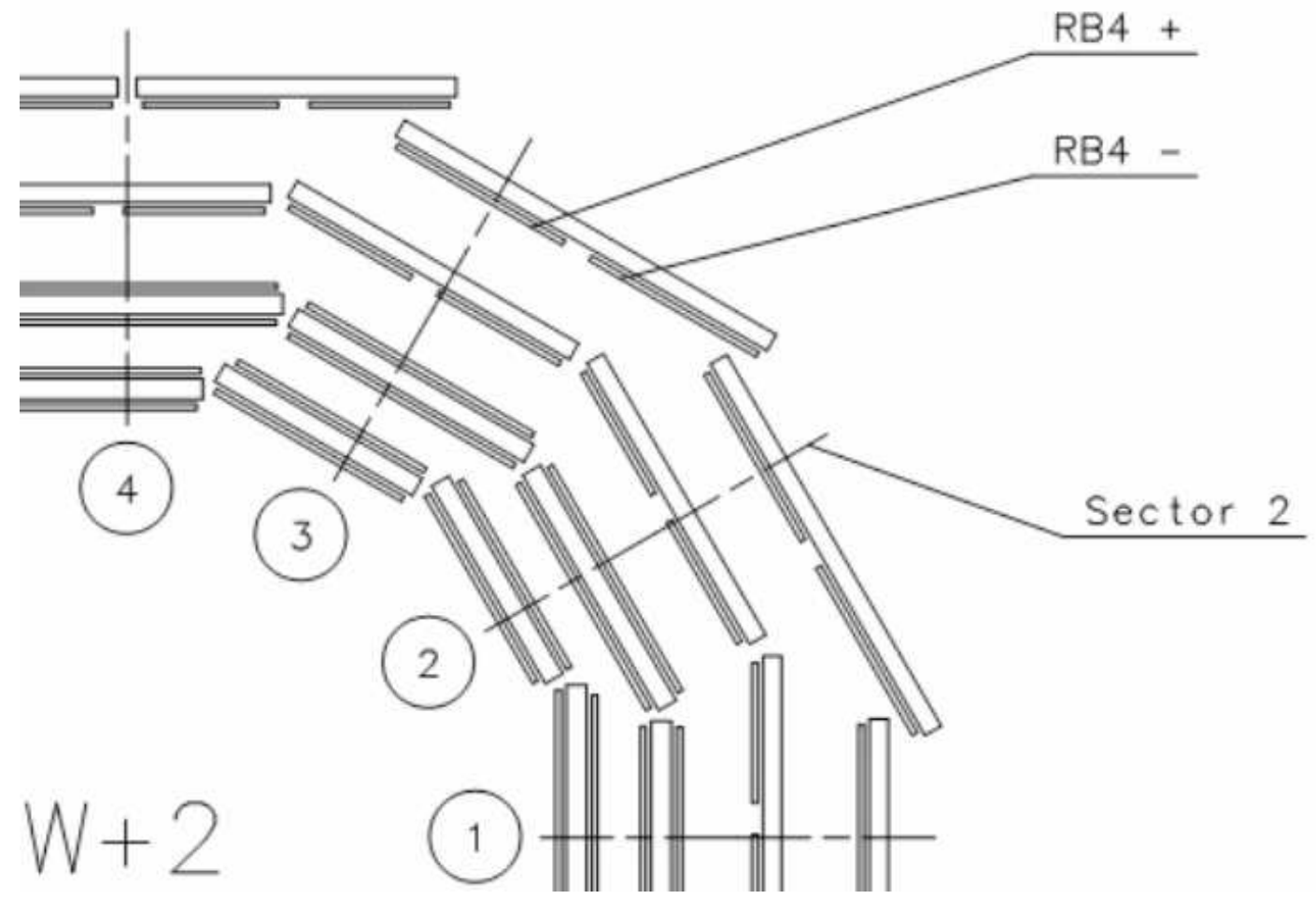

Figure 2.11: Location of resistive plate chamber in CMS barrel region.

\subsubsection{Trigger}

The design luminosity of the LHC is $10^{9} \mathrm{~Hz}$ proton-proton collisions. This combined with the massive number of channels in CMS as detailed in the previous few pages means that it is not possible to store all the data being produced by the CMS detector. Instead a trigger system is used to determine which bunch crossings are interesting and should be saved. The trigger system uses all the previously described subsystems to make its determination.

The trigger was constructed in two parts a level 1 (L1) and high level trigger 
(HLT). The L1 trigger consists of programmable electronics located on or right next to the detector. The HLT on the other hand is software based and located in a server room next to the control room of CMS. The HLT software uses about 1000 processors for its calculations. A schematic of the trigger's logic flow is shown in Figure 2.12. During the trigger processing of an event, that event is stored in a buffer before it is ultimately stored or thrown away.

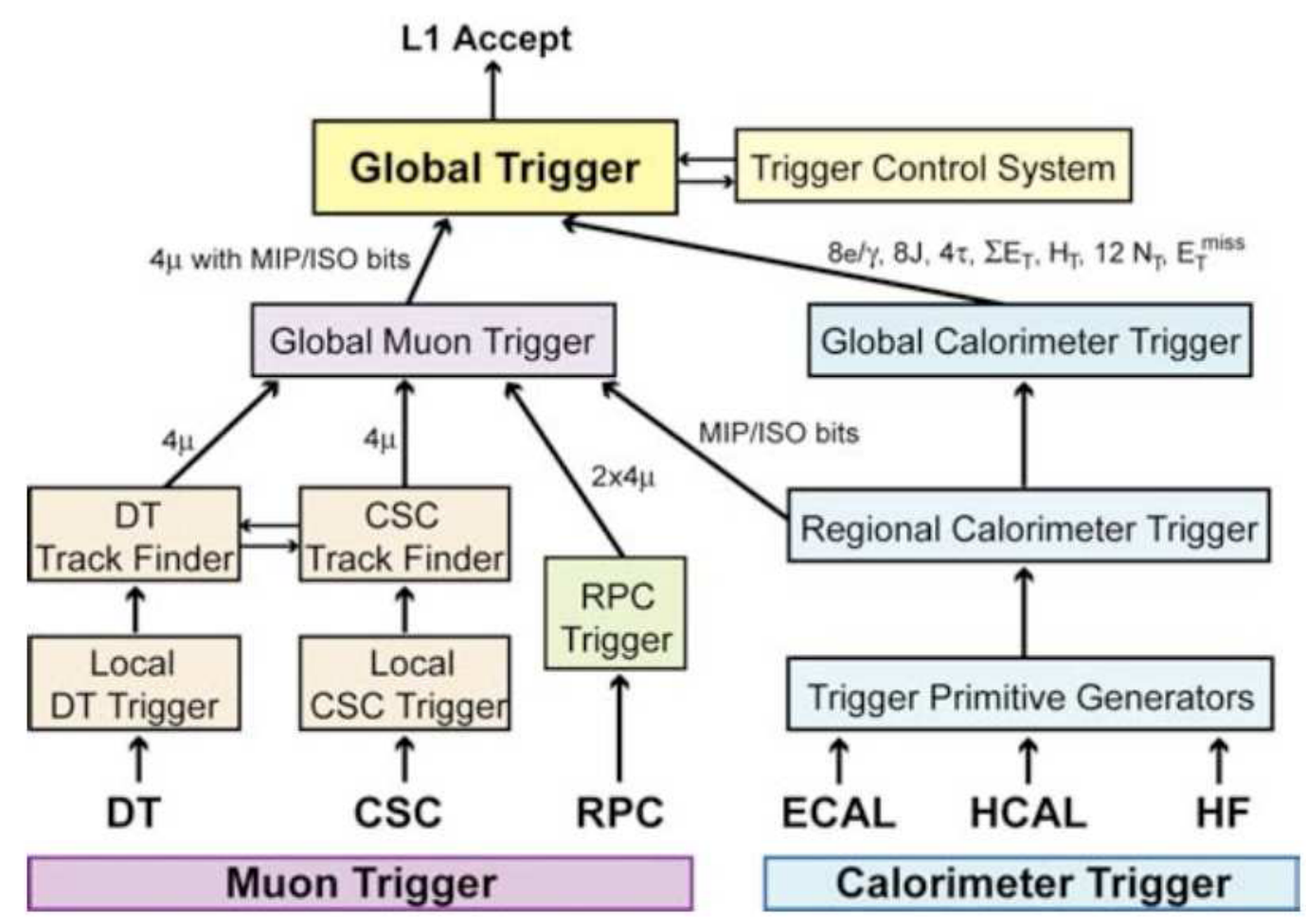

Figure 2.12: Diagram of information flow in the Level 1 trigger chain in CMS.

The L1 trigger receives information from all the subdetectors but the inner tracker. The inner tracker is to far within CMS and produces to much data to move and process its signal fast enough to use. The L1 trigger designed to operate at 100 $\mathrm{kHz}$ reducing the event rate by a factor of 400. As the trigger used in the analysis described by this paper is the muon trigger it is described in more detail here.

The muon L1 trigger combines information from the DT and CSC for track 
finding along with the RPC. This data is collected by the global muon trigger (GMT). The GMT also receives information from the concurrently running calorimeter L1 stream from the regional calorimeter trigger $(\mathrm{RCT})$. This allows for more complex muon trigger including isolation variables along with simple identification and momentum. The GMT sends its information to the global trigger (GT) which can then issue an L1 accept (L1A). An L1A causes all the subdetectors to send the event information for that bunch crossing stored in their buffers to the global data acquisition system (DAQ). The DAQ provides two data streams, one for use by the HLT and another for storage based upon the HLT's decision.

While the L1 trigger can be modified it is located underground near the detector as electronics, making any changes difficult. The HLT on the other hand is above ground software and was designed to change over time; different changes are called menus. The HLT also has access to the full detector readout, and can therefore make more complicated calculations. It is designed to produce a $100 \mathrm{~Hz}$ output for a final reduction of the event rate by $4 \times 10^{5}$. The HLT menu's are composed of primary datasets (PDs) which define specific types of event content. Used in the analysis described by this paper were the single muon PD and the double muon PD, composed of events passing a single muon trigger requirement and a double muon trigger requirement respectively. 


\section{CHAPTER 3 \\ HEAVY MAJORANA NEUTRINO SEARCH}

\subsection{Introduction}

As detailed in Chapter 1.2, the non-zero masses of the neutrinos have been recently confirmed from oscillation experiments and are arguably the first evidence for physics beyond the SM. The tiny values of the observed neutrino mass aggravate the lack of an understanding of mass generation for elementary particles, but could be solved by the "see-saw" mechanism, where the new heavy state is a new Majorana mass neutrino.

For neutral particles, there is an alternative way to write down the Dirac equation for a fermion where all the $\gamma$ matrices are real [2]. This equation proposed by Ettore Majorana produces particles which are their own anti-paritcles [27]. As a new heavy neutrino with a Majorana nature would be its own anti-particle; this would allows for decays which violate lepton number conservation by two units. Searches for heavy Majorana neutrinos at hadron colliders have been considered by many authors $[28,29,30,31,32]$, and the interest for the LHC has been lately renewed [33, 34, 35]. This search follows a recent study for the $14 \mathrm{TeV}$ LHC [33], which takes a model-independent phenomenological approach, assuming that the heavy neutrino mass $M_{N}$ and $V_{\ell N}$, the mixing element describing the mixing between the heavy Majorana neutrino and the SM neutrino $\nu_{\ell}$ of flavor $\ell$, are free parameters. Previous direct searches for heavy Majorana neutrinos have been reported by the DELPHI [36] and L3 [37] collaborations. They set limits on the mixing element squared for Majorana neutrino masses below $90 \mathrm{GeV} / \mathrm{c}^{2}$.

This thesis describes an analysis consisting of a detailed search for the resonance production of a heavy Majorana neutrino as shown by the Feynam in Figure 3.1. The analysis use data from the the CMS detector located at the LHC. 
The complete 2011 run period is used which provides $4.96 \mathrm{fb}^{-1}$ of data. The analysis proceeds with a search for events from the 2011 dataset containing same sign isolated dimuons $\left(\mu^{ \pm} \mu^{ \pm}\right)$and two jets. Also studied but not covered in this thesis is the same sign dielectron signature. Standard Model processes creating this signature are very small. The background is dominated by processes involving either one isolated prompt lepton and one non-prompt lepton or two non-prompt leptons. Throughout this document the former type of lepton is referred to as a "fake lepton." There are many sources of fake lepton events, as fake leptons can be produced in any hadronic shower decay (called a jet). As an example, in the first case the single isolated prompt lepton may come from a decaying $\mathrm{W}$ boson which is produced in conjunction with several jets. One of those jets could then fake an electron; this type of event is referred to as $\mathrm{W}+$ jets.

Figure 3.2 shows the cross section for heavy Majorana neutrino production and decay via the process in Figure 3.1 as a function of Majorana neutrino mass for $\left|V_{\ell N}\right|^{2}=1.0$. The plot shows the results from the two independent event generators, del Aguila et al. [34] and Han and Zhang [33].

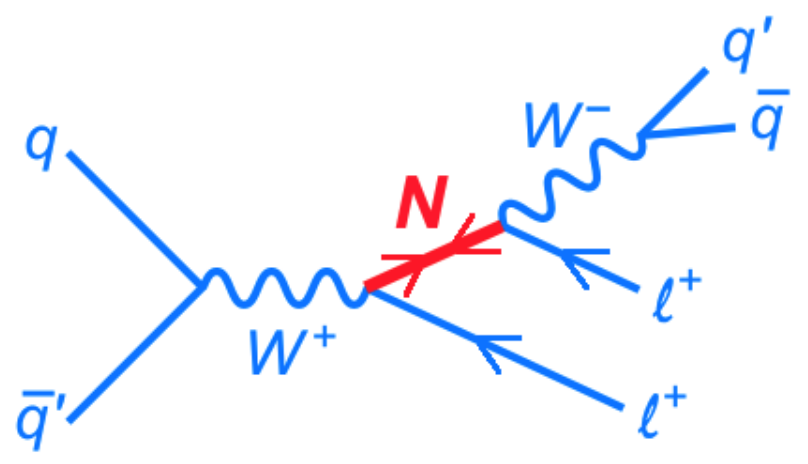

Figure 3.1: The Feynman diagram for resonance production of a Majorana neutrino $(N)$. The charge-conjugate diagram results in a $\ell^{-} \ell^{-} q \bar{q}^{\prime}$ final state. 


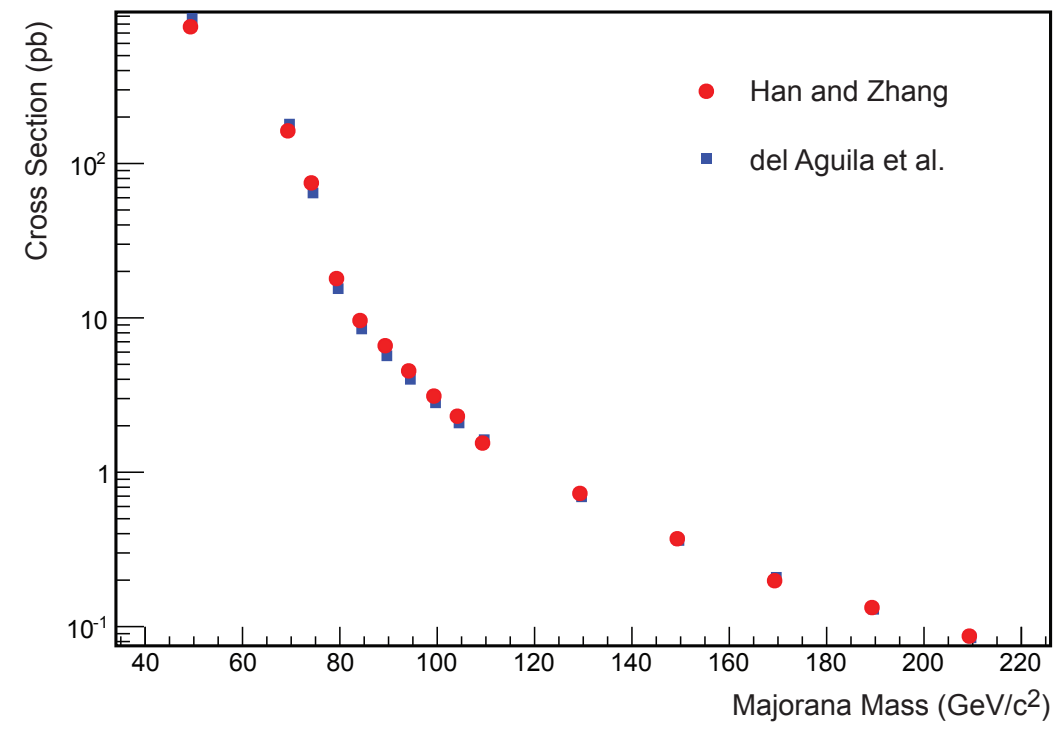

Figure 3.2: The cross section for resonance production of a Majorana neutrino as a function of Majorana neutrino mass from two independent event generators.

\subsection{Event Generation and Simulation}

Searches for new physics in high energy particle physics experiments requires a good understand of the way the detector records the already understood physics processes of the Standard Model. Similarly the new physics often must be modeled to provide information on how to search through the data. While calculations of the tree level interactions that form the basis of the processes being studied are relatively straightforward, there are many additional complications when considering the signal actually read out by the detector.

At just the tree level, both the incoming and outgoing particles may radiate, referred to as initial state radiation (ISR) and final state radiation (FSR). Additionally all charge particle radiate when curving in a magnetic field such as is produced by CMS's solenoid, and particles lose energy when passing through the detector material. The showers from particles decaying are intended to be detected in the calorimeters but no calorimeter is $100 \%$ efficient. This is particularly the case for a 
sampling calorimeter such as HCAL. These factors combine to make precise calculation of the results from a specific process impossible to analytically calculate, and in fact many of the factors are driven by probability so must be studied through the use of large statistical samples providing a distribution of the possible outcomes from the process.

Instead, tools have been developed to simulate various types of collision events. These simulations can be compared to (and tuned to match) real data from an experiment like CMS. This type of simulation algorithm is referred to as a Monte Carlo, MC, simulation in reference to the probabilistic nature of the data being simulated. It is used to produce large simulated datasets allowing for the preparation of what to expect in real collision data.

Even before an experiment is built allowing for data collection, MC simulations can provide guidance in the detector design and in the development of the software tools that will eventually be used for physics analysis. Once data is available, simulations help in the development of an analysis tailored to a specific signal being searched for in the data. Standard MC simulation has two step event generation where tree level interactions are calculated, then events are simulated where the outgoing particle's detector interactions are included.

\subsubsection{Event Generation}

The first step of MC simulation, event generation, produces the final state particles from an interaction of two colliding particles. The final state particles are provided with their correct kinematical properties. These final state particles are either produced for a particular hard scatter or for a particular set of processes. Their kinematical properties are determined by matrix element calculations. The matrix calculations consider all possible tree level diagrams up to a preset level of precision, either specific to the generator or defined by the user. The generator step 
also includes ISR, FSR, and the decay of the non-stable final state particles. This includes hadronic showers of colored particles called "jets." How FSR and ISR are handled by different generators can affect the topology of jets in the final output of the generator. The two generators used in this analysis provide an example of these differences.

One of the most commonly used generators, PYTHIA [38], does not include ISR or FSR at the matrix element level calculation. It only does the hard scatter process calculations. Following that it uses a very successful model for implementing the hadronization of colored particles called the "Lund string model." The Lund model worked very well for the $e^{+} e^{-}$colliders PETRA and PEP, and was the first real successful implementation of hadronic showers. It uses a string to model the potential between quark pairs. As the quarks separate, the string stretches, representing an increase in potential energy. When the tension is great enough the string breaks, forming new quark pairs. Radiating gluons are represented as kinks in the string which increase the tension. This does a very good job with jet substructure, but not with jet multiplicity.

Improving jet multiplicity calculations has become important with the advent of modern hadron-hadron colliders such as the LHC and previously the Tevatron at Fermilab. To this end, several generators have been developed to perform matrix element calculations that include FSR and ISR. Their output can then be allowed to develop via the PYTHIA style shower generators. This style of generator introduces a new parameter in order to keep the calculations time practical. A scale, $Q_{c u t}$, is defined such that a radiating particle below that scale is not considered part of the full matrix element calculation.

The MC simulations of the Majorana neutrinos searched for in this analysis used the ALPGEN [39] generator. ALPGEN is an example of a generator that uses 
exact calculations to the first order in perturbative QCD in order to determine the final state partons. This calculated hard scatter process is then added to final state gluon radiation. The matrix element calculation used the ALPHA algorithm [40]. The ALPHA algorithm uses a Greens' function generator for the hard scatter's associated Lagrangian. This allows ALPGEN to preserve the full color and spin information for the process.

Regardless of the type of generator being used, to correctly generate the particles kinematic spectra across multiple simulated events, the process's cross section $(\sigma)$ must be known. This would be fine if the colliders could accelerate quarks rather than colorless hadrons as perturbative QCD can predict $(\sigma)$ for $q_{i}+q_{j}=\Sigma_{n} q_{n}$ where $q_{i}$ and $q_{j}$ are partons with known momenta and $q_{n}$ are the $\mathrm{n}$ final state particles. However, only the accelerated hadrons (in the case of the LHC these are protons) have known momentum, not the partons within the protons. The solution to this problem is the use of parton distribution functions (PDFs). These functions are derived experimentally, and describe the distribution of the parton's momentum to its constituent partons.

There are many different choices of PDFs which vary in how they have been determined from experimental data. They all use data from a raft of different experiments including data from mature hadronic colliders where the processes are well understood along with fixed target experiment scattering experiments. The data being produced from the LHC collisions provides a very large amount of minimum bias events in a new high energy regime. Studies of these events have provided the ability to begin to put new constraints on these PDFs. The data from these sources is fit to functions which depend upon the fraction of the hadrons momentum, $x$, carried by a parton.

Equation 3.1 shows how the p-p cross section can then be calculated for any 
process,

$$
\sigma\left(P_{1}, P_{2}\right)=\Sigma_{i, j} \int f_{1}\left(x_{1}, Q^{2}\right) \times f_{2}\left(x_{2}, Q^{2}\right) \times \sigma_{i j}\left(x_{1}, x_{2}, Q^{2}\right) d x_{1} d x_{2} d Q^{2}
$$

Here $P_{1}$ and $P_{2}$ are the momenta of the protons, $x_{1}$ and $x_{2}$ are momenta fraction carried by the partons in the hard scatter, $f_{1}$ and $f_{2}$ are the PDFs for the partons, $Q$ is the total momentum being transferred between the partons, $\sigma_{i}$ is the cross section of the hard scatter process being calculated, and the sum is taken over all the partons that could contribute to the process being studied.
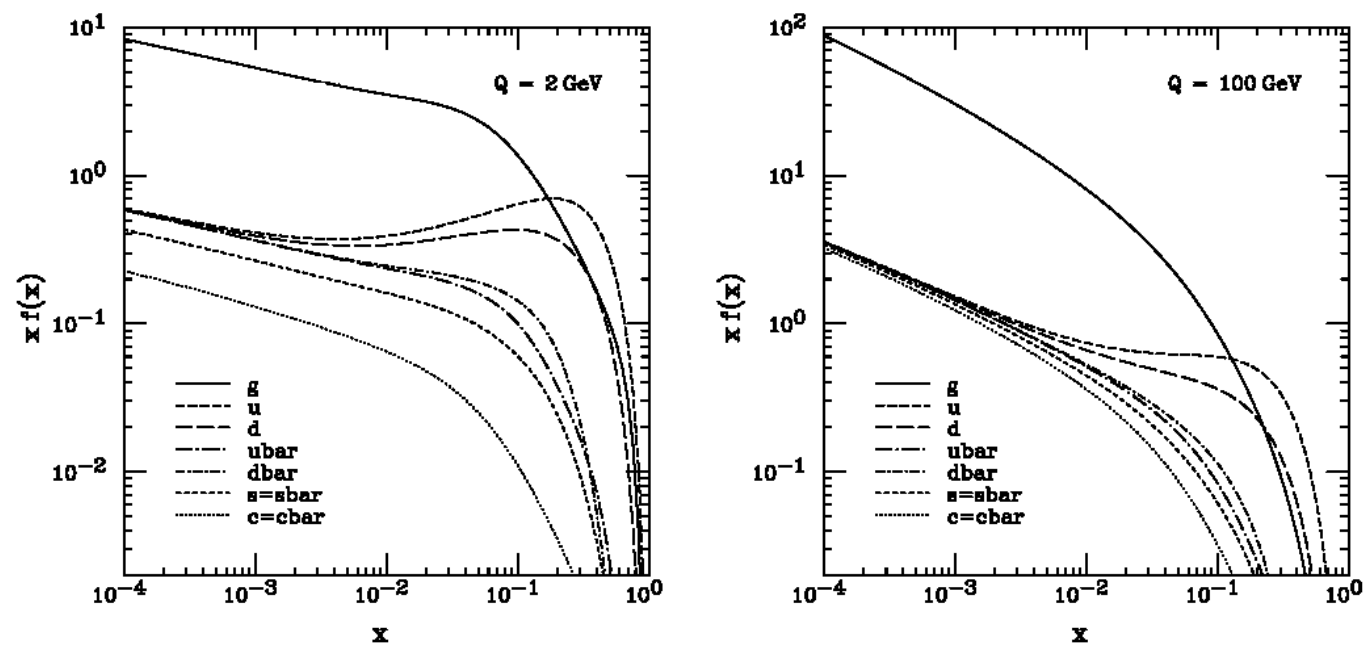

Figure 3.3: Parton Distribution Functions from the CTEQ collaboration for two values of interaction scale $\mathrm{Q}$.

Figure 3.3 shows plots of the PDF's from the CTEQ collaboration [41, 42]. From this figure, where the horizontal axis is the fraction of the total proton momentum carried by the parton, $x$, and the vertical axis shows the product of the parton momentum fraction and the $\mathrm{PDF}$, it can be seen that for greater $Q$, a larger fraction of the momentum is carried by the sea quarks. When the LHC was being designed this fact allowed for $p-p$ collisions to be used rather than the $p-\bar{p}$ collisions used 
at the lower energy Tevatron collider.

\subsubsection{Event Simulation}

The actual signals recorded by a detector are the many decay products of the generated final state particles in thousands of electronic channels across many different subdetectors. Full understanding of the data must include simulation of the detectors response. For this, a detailed computer model must be developed including precise detector geometry and accurate representation of the physics interactions of these particles within the detector's material. The primary tool for this type of simulation in HEP and the one used by CMS is GEANT4 [43].

GEANT4 is a highly configurable software toolkit for the computer design and simulation of detectors. Users may specify all the physical characteristics of a detector and then (to a user defined level of intricacy) it will step particles through the detector simulating the full physics interactions. The software considers all possible electromagnetic, optical, or hadronic physics interactions along with particle decay at each "step" of the particle's through the detector.

GEANT4 works in an iterative way moving particles in simulated geometrical steps through the detector. At each iterative step an algorithm chooses which process is appropriate and applies it to the particle. The algorithm only applies processes that are relevant to the particle while the stepping process is generic to all particles. The detector is not static during runs as there will be previously unanticipated detector noise, as well as malfunctions that may require some channels to not be read out. GEANT4 allows for the detector simulation be adjusted to reflect this.

As mentioned the user can define a level of intricacy in the detector simulation's steps. Part of this is the ability to declare material "sensitive." This requires that the software remember more details of the particles entering or produced in 
that material. This allows for the creation of "hits" to this material representing the hits to the sensitive material in the actual CMS detector. From there collections of hits can be formed into "digis" just as is done with the actual electronic readouts. Digis from various subdetectors can be added together to form full simulated events. Multiple events can even be combined to simulate the results from multiple interactions occurring during a bunch crossing, called pile up.

At this point the simulated events in digi form are in the same format as the digis coming from actual collisions. Therefore, the same software for event reconstructions is used from this point on. There are detector specific pieces of software for reconstructing high level physics objects. "High level physics objects" is the generic software term for real particles and features coming from the collisions: electrons, jets, photons, muons, and missing transverse energy $\left(E_{\mathrm{T}}^{\mathrm{miss}}\right)$.

This analysis concerns itself primarily with reconstructed muons and jets, which are discussed in the following sections.

\subsection{Muon Reconstruction}

Muons are reconstructed offline from a combination of silicon tracker hits (tracker track) and muon chamber hits (standalone-muon track). There are three types of muons that can be reconstructed:

- Tracker Muons: All tracker tracks fitting some simple kinematic requirements are considered as muons.

- Standalone Muons: Muons with only a track in the muons system.

- Global Muons: These start from a standalone-muon and are matched to a tracker track. 
Tracker muons and standalone muons are first independently reconstructed. Global muons which are then reconstructed by matching each of the standalonemuon tracks to an independently reconstructed tracker track. The complete set of hits contained in the matched tracks from both detector systems is then refit for a global-muon track. A more complete description of all three types of muons is given in Reference [44].

\subsection{Jet Reconstruction}

Jets are reconstructed from combinations of HCAL and ECAL cells located in the same region of $(\eta, \phi)$ space; these cell combinations are referred to as calorimeter "towers". Towers are clustered together using the anti- $k_{T}$ clustering algorithm [45]. A jet "cone" is visualized with its vertex located at the collision vertex, and its central axis co-aligned with an energy-weighted axis through the center of the jet. Jet clustering algorithms typically make use of such cones to determine what tracks or towers to include in the total jet energy/momentum calculation; a cone with angular radius $\Delta R=\sqrt{(\Delta \eta)^{2}+(\Delta \phi)^{2}}=0.5$ was used to reconstruct jets for this analysis. The anti- $k_{T}$ algorithm attempts to combine the two general types of jet clustering algorithms: iterative cone based algorithms and sequential recombination clustering algorithms such at the $k_{t}$ and Cambridge/Aachen algorithms [46]. Iterative cone algorithms have the advantage of geometrically intuitive clustering (physical cones) and are based around hard particle/pseudojets; they however don't have a clear method of dealing with overlapping jets such as can be found in the high luminosity and energy environment at the LHC. The sequential recombination algorithms are much better at handling these high jet multiplicity environments, but suffer in that they can create geometrically unnatural jet shapes and can prefer clustering soft particles above hard particles.

Jets are required to pass the following quality requirements, which are intended 
to suppress backgrounds from calorimetric noise:

1. A jet's energy must not have a single tower contributing more than $90 \%$ of its overall energy.

2. The electromagnetic fraction of the jet (the fraction of the jet's energy that is measured in the electromagnetic calorimeter) must exceed $1 \%$.

3. The fraction of the jet's energy that is measured in a single photodetector in the hadron calorimeter (which spans multiple towers) must be less than $98 \%$.

In addition, after jet identification and before any selection on the jet $p_{T}$ is performed, jet energy scale corrections are applied. The corrections are factored into stages; currently two stages of corrections are applied. The first stage levels the jet response as a function of pseudorapidity across subdetectors, and the second stage levels the jet response as a function of $p_{T}$. These corrections are derived currently from MC truth; therefore the data are subjected to a third residual correction in the relative energy scale that compensates for known differences between the MC and data [47].

\subsection{Analysis}

\subsubsection{Data and Monte Carlo Samples}

The heavy Majorana neutrino production and decay process is simulated using the event generator described in Reference [34] and implemented in ALPGEN. The production process simulated is the resonant production of a Majorana neutrino (see Figure 3.1) for proton-proton collisions at $7 \mathrm{TeV}$. The output of the ALPGEN generator was stored in the Les Houches 1.0 format [48]. These data sets were then unweighted using PYTHIA [38] version 6.4 to generate events for each neutrino mass. The generated event files were interfaced with CMS Software, CMSSW, version 4_2_4 
where parton showering, vertex smearing, GEANT4 detector simulation, digitization of simulated electronics signal, and reconstruction were performed. The Monte Carlo samples are reweighted to ensure the proper simulation of the number of interactions per crossing. The list of official production Monte Carlo samples and the cross section as a function of Majorana neutrino mass, are shown in Table 3.1 (the abbreviations used in the table are specified in Table 3.3), using a Majorana neutrino muon coupling value $S_{\mu \mu}=\left|V_{\mu N}\right|^{2}=1$.

The Monte Carlo samples used for background estimation and validation of the data-driven background estimation methods are listed in Table 3.2. Samples used to estimate the number of events from standard model processes include diboson production, $t \bar{t} W$ production, and same sign $W$ pair production via double $W$-strahlung and double parton scattering. The $t \bar{t}, W+$ jets, and QCD samples are used to validate the fake background estimation method. The Monte Carlo samples are reweighted to ensure the proper simulation of the number of interactions per crossing.

The data used in this analysis is comprised of both the 2011A and 2011B Run CMS datasets, as listed in Table 3.4. The total integrated luminosity is $4.96 \pm$ $0.11 \mathrm{fb}^{-1}$.

CMS data is broken up into so-called primary datasets, PD, which are organized based upon the type of trigger which selected the event. Data from two of the CMS primary datasets is used. For the heavy Majorana neutrino signal selection the lowest available unprescaled double muon trigger from the DoubleMu primary dataset is used. The triggers used are:

- HLT_DoubleMu7 for runs 160404 to 163869

- HLT_Mu13_Mu8 for runs 165071 to 178380

- HLT_Mu17_Mu8 for runs 178424 to 180252 
Table 3.1: Summary of Majorana neutrino signal Monte Carlo samples generated with $\left|V_{\mu N}\right|^{2}=1$ including the calculated cross section as a function of Majorana neutrino mass.

\begin{tabular}{|c|c|c|c|}
\hline $\begin{array}{l}\text { Mass } \\
\left(\mathrm{GeV} / \mathrm{c}^{2}\right)\end{array}$ & Dataset Name & $\begin{array}{c}\text { Cross } \\
\text { Section (pb) }\end{array}$ & $\int \mathcal{L} d t\left(\mathrm{pb}^{-1}\right)$ \\
\hline 50 & /NToMuMu_M-50_7TeV_Alpgen/Fall11 & $866.4 \pm 1.1$ & 57.70 \\
\hline 60 & /NToMuMu_M-60_7TeV_Alpgen/Fall11 & $492.5 \pm 0.7$ & 101.52 \\
\hline 70 & /NToMuMu_M-70_7TeV_Alpgen/Fall11 & $171.3 \pm 0.3$ & 291.81 \\
\hline 75 & /NToMuMu_M-75_7TeV_Alpgen/Fall11 & $64.70 \pm 0.10$ & 768.11 \\
\hline 80 & /NToMuMu_M-80_7TeV_Alpgen/Fall11 & $15.21 \pm 0.02$ & 3286.9 \\
\hline 85 & /NToMuMu_M-85_7TeV_Alpgen/Fall11 & $(8242 \pm 6) \times 10^{-3}$ & 6066.0 \\
\hline 90 & /NToMuMu_M-90_7TeV_Alpgen/Fall11 & $(5601 \pm 2) \times 10^{-3}$ & 8604.5 \\
\hline 95 & /NToMuMu_M-95_7TeV_Alpgen/Fall11 & $(3915 \pm 1) \times 10^{-3}$ & 12770 \\
\hline 100 & /NToMuMu_M-100_7TeV_Alpgen/Fall11 & $(2787 \pm 1) \times 10^{-3}$ & 17938 \\
\hline 105 & /NToMuMu_M-105_7TeV_Alpgen/Fall11 & $(2067.7 \pm 0.6) \times 10^{-3}$ & 24179 \\
\hline 110 & /NToMuMu_M-110_7TeV_Alpgen/Fall11 & $(1590.2 \pm 0.5) \times 10^{-3}$ & 31250 \\
\hline 120 & /NToMuMu_M-120_7TeV_Alpgen/Fall11 & $(1017.6 \pm 0.3) \times 10^{-3}$ & 49126 \\
\hline 130 & /NToMuMu_M-130_7TeV_Alpgen/Fall11 & $(690.6 \pm 0.2) \times 10^{-3}$ & 69791 \\
\hline 150 & /NToMuMu_M-150_7TeV_Alpgen/Fall11 & $(354.8 \pm 0.1) \times 10^{-3}$ & 140900 \\
\hline 170 & /NToMuMu_M-170_7TeV_Alpgen/Fall11 & $(203.93 \pm 0.06) \times 10^{-3}$ & 236336 \\
\hline 190 & /NToMuMu_M-190_7TeV_Alpgen/Fall11 & $(126.57 \pm 0.04) \times 10^{-3}$ & 395030 \\
\hline 210 & /NToMuMu_M-210_7TeV_Alpgen/Fall11 & $(83.011 \pm 0.024) \times 10^{-3}$ & 569804 \\
\hline
\end{tabular}

Note: The abbreviation Fall11 is used for Fall11-PU_S6_START42_V14B/AODSIM and NToMuMu for MajoranaNeutrinoToMuMu. 
Table 3.2: Summary of standard model Monte Carlo datasets used.

\begin{tabular}{|c|c|c|c|}
\hline Dataset & Dataset Name & $\sigma(p b)$ & $\int \mathcal{L} d t\left(\mathrm{pb}^{-1}\right)$ \\
\hline$W^{+} W^{-}$ & /WW_TZ2_7_p_tauola/F11 & 43 & $9.83 \times 10^{4}$ \\
\hline$W Z$ & /WZ_TZ2_7_p_tauola/F11 & 18.2 & $2.34 \times 10^{5}$ \\
\hline$Z Z$ & /ZZ_TZ2_7_p_tauola/F11 & 5.9 & $7.10 \times 10^{5}$ \\
\hline$W \gamma$ & /WGstarToLNu2Mu_TuneZ2_7m-t/S11a & 1.60 & $6.23 \times 10^{4}$ \\
\hline$W^{+} W^{+}$ & /PhysicsProcesses_WplusWplus_42x_v1 & 0.165 & $8.11 \times 10^{5}$ \\
\hline$W^{-} W^{-}$ & /PhysicsProcesses_WminusWminus_42x_v1 & 0.0551 & $3.18 \times 10^{6}$ \\
\hline $\operatorname{dp} W^{ \pm} W^{ \pm}$ & /DoublePartonWWFastSim_CMSSW425PUv1 & 0.38 & $1.06 \times 10^{6}$ \\
\hline$t \bar{t}$ & /TTJets_TuneZ2_7m-t/F11 & 154 & $2.40 \times 10^{4}$ \\
\hline$t\left(t W^{-}\right)$ & /T_TuneZ2_tW-channel-DR_7p-t/F11 & 5.3 & $1.54 \times 10^{5}$ \\
\hline $\bar{t}\left(\bar{t} W^{+}\right)$ & /Tbar_TuneZ2_tW-channel-DR_7p-t/F11a & 5.3 & $1.53 \times 10^{5}$ \\
\hline$t(s$-channel $)$ & /T_TuneZ2_s-channel_7p-t/F11 & 2.72 & $9.56 \times 10^{4}$ \\
\hline $\bar{t}(s$-channel $)$ & /Tbar_TuneZ2_s-channel_7p-t/F11 & 1.49 & $9.26 \times 10^{4}$ \\
\hline$t(t$-channel $)$ & /T_TuneZ2_t-channel_7p-t/F11 & 42.6 & $9.16 \times 10^{4}$ \\
\hline $\bar{t}(t$-channel $)$ & /Tbar_TuneZ2_t-channel_7p-t/F11 & 22 & $8.84 \times 10^{4}$ \\
\hline$t \bar{t} W$ & /TTbarInclWIncl_TuneZ2_7m-t/F11 & 0.153 & $2.29 \times 10^{6}$ \\
\hline$W+$ jets & /WJetsToLNu_TuneZ2_7m-t/F11 & $3.13 \times 10^{4}$ & $2.6 \times 10^{3}$ \\
\hline$Z+$ jets & /ZMuMu_Pt-0to15_TZ2_7_p/S11 & 4281 & 48.9 \\
\hline$Z+$ jets & /ZMuMu_Pt-15to20_TZ2_7_p/S11 & 145.1 & $1.50 \times 10^{3}$ \\
\hline$Z+$ jets & /ZMuMu_Pt-20to30_TZ2_7_p/S11 & 130.5 & $1.26 \times 10^{3}$ \\
\hline$Z+$ jets & /ZMuMu_Pt-30to50_TZ2_7_p/S11 & 84.0 & $1.85 \times 10^{3}$ \\
\hline$Z+$ jets & /ZMuMu_Pt-50to80_TZ2_7_p/S11 & 32.2 & $3.31 \times 10^{3}$ \\
\hline$Z+$ jets & /ZMuMu_Pt-80to120_TZ2_7_p/S11 & 9.98 & $1.10 \times 10^{4}$ \\
\hline $\mathrm{QCD}$ & /QCD_Pt-15to20_MuPt5_TZ2_7_p/F11 & $5.79 \times 10^{8}$ & 1.51 \\
\hline $\mathrm{QCD}$ & /QCD_Pt-20to30_MuPt5_TZ2_7_p/F11 & $2.36 \times 10^{8}$ & 7.51 \\
\hline $\mathrm{QCD}$ & /QCD_Pt-30to50_MuPt5_TZ2_7_p/F11 & $5.31 \times 10^{7}$ & 18.3 \\
\hline QCD & /QCD_Pt-50to80_MuPt5_TZ2_7_p/F11 & $6.35 \times 10^{6}$ & 77.3 \\
\hline QCD & /QCD_Pt-80to120_MuPt5_TZ2_7_p/F11 & $7.85 \times 10^{5}$ & 291 \\
\hline $\mathrm{QCD}$ & /QCD_Pt-120to150_MuPt5_TZ2_7_p/F11 & $9.30 \times 10^{4}$ & 1772 \\
\hline QCD & /QCD_Pt-150_MuPt5_TZ2_7_p/F11 & $4.76 \times 10^{4}$ & 1409 \\
\hline
\end{tabular}

Abbreviations are defined in Table 3.3 
Table 3.3: Abbreviations used in Table 3.2.

\begin{tabular}{cc}
\hline \hline & Abbriviations Used \\
\hline$/$ F11 & /Fall11-PU_S6_START42_V14B-v1/AODSIM \\
/F11a & /Fall11-PU_S6_START42_V14B-v2/AODSIM \\
/S11 & /Summer11-PU_S3_START42_V11-v2/AODSIM \\
/S11a & /Summer11-PU_S4_START42_V11-v1/AODSIM \\
/ZMuMu & /ZJetToMuMu \\
MuPt5 & MuPt5Enriched \\
$7 \mathrm{p}-\mathrm{t}$ & 7TeV-powheg-tauola \\
$7 \mathrm{~m}-\mathrm{t}$ & 7TeV-madgraph-tauola \\
TZ2_7_p & TuneZ2_7TeV_pythia6 \\
\hline
\end{tabular}

Table 3.4: Summary of 2011 datasets used.

\begin{tabular}{ll}
\hline \hline Dataset & Run Range \\
\hline /DoubleMu/Run2011A-May10ReReco-v1/AOD & $160329-163869$ \\
/DoubleMu/Run2011A-PromptReco-v4/AOD & $165071-167784$ \\
/DoubleMu/Run2011A-05Aug2011-v1/AOD & $170053-172619$ \\
/DoubleMu/Run2011A-PromptReco-v6/AOD & $172620-175770$ \\
/DoubleMu/Run2011B-PromptReco-v1/AOD & $175832-180252$ \\
/SingleMu/Run2011A-May10ReReco-v1/AOD & $160329-163869$ \\
/SingleMu/Run2011A-PromptReco-v4/AOD & $165071-167151$ \\
/SingleMu/Run2011A-05Aug2011-v1/AOD & $170053-172619$ \\
/SingleMu/Run2011A-PromptReco-v6/AOD & $172620-175770$ \\
/SingleMu/Run2011B-PromptReco-v1/AOD & $175832-180252$ \\
\hline
\end{tabular}


The corresponding integrated luminosities for these triggers are $0.2153 \mathrm{fb}^{-1}, 3.860 \mathrm{fb}^{-1}$, and $0.8847 \mathrm{fb}^{-1}$, respectively. The efficiency of these triggers is discussed in Section 3.5.2.

For the fake rate background estimation and the tag and probe study, events collected using single muon triggers were used to calculate the fake rates and muon selection efficiencies. The triggers used were:

- HLT_Mu8

- HLT_Mu8_Jet40

- HLT_Mu15

\subsubsection{Event Selection}

The signal signature is characterized by two muons and an accompanying $W$ boson. Because of the Majorana nature of the heavy neutrino considered here, both opposite-sign and same-sign muons are produced. In this note we concentrate on the same-sign dimuon signature since this final state has very low standard model backgrounds. In addition to these muons, the Majorana Neutrino also produces an accompanying $W$ boson during its decay. This analysis looks for signatures in which the $\mathrm{W}$ decays to two jets, as this allows the consideration of final states without light neutrinos.

The selection criteria for each of the two like sign muons is as follows:

- $\chi^{2} / n d o f<10$

- $d_{0}$ (the distance of closest approach to the origin of the muon's projected path in the xy-plane) relative to the primary vertex mentioned above $<0.1 \mathrm{~mm}$

- $d_{z}$ (the distance of closest approach to the origin of the muon's projected path along the z-axis) within $0.1 \mathrm{~cm}$ of primary vertex 
- Number of hits in the tracker $\geq 11$

- Number of hits in the muon system $\geq 1$

- Global and tracker muon types

- $p_{\mathrm{T}}>10 \mathrm{GeV} / \mathrm{c}(>20 \mathrm{GeV} / \mathrm{c}$ for one muon)

- $|\eta|<2.4$

- isolated muon (where isolation values are all calculated in a $\Delta R$ cone of 0.3 excluding the muons contribution)

- sum ECAL deposits $<4 \mathrm{GeV}$

- sum HCAL deposits $<6 \mathrm{GeV}$

- relative isolation $($ RelIso) $<0.1$ (defined as the sum of the energy deposits in the ECAL, HCAL, and tracker divided by the $p_{\mathrm{T}}$ of the muon)

Isolation is corrected for pileup for both muons and electrons using energy density $\rho$ in the event [49]. The quantity of $\pi \rho r^{2}$ is subtracted from the isolation value. The value of $\rho$ depends on the pileup condition of each event and is parametrized in in 5 bins in pseudorapidity.

The two jets in the event having passed the quality cuts and after jet energy scale corrections have been applied are required to have a $p_{\mathrm{T}}>30 \mathrm{GeV} / \mathrm{c}$ and $|\eta|<$ 2.5 and be well separated from the muon candidates $(\Delta R>0.4)$.

After the requirement of two same sign muons and two jets described above, events were required to satisfy the following criteria:

- dimuon mass $>5 \mathrm{GeV} / \mathrm{c}^{2}$

- events with a 3rd opposite sign muon that combines with one of the candidate muons to have a mass within $76-106 \mathrm{GeV} / \mathrm{c}^{2}$ are excluded 
Finally, the events are required to have missing transverse energy $\left(E_{\mathrm{T}}^{\mathrm{miss}}\right)$ less than $50 \mathrm{GeV}$. This requirement suppresses backgrounds such a $t \bar{t}$ and $W+$ jets, which have significant real $E_{\mathrm{T}}^{\text {miss }}$ in the final state. Events with $E_{\mathrm{T}}^{\text {miss }}>50 \mathrm{GeV}$ are used as a control sample to cross check the background prediction.

The double muon trigger selection efficiencies are discussed in detail in Reference [50]. As this analysis uses the same muon selection requirements, their results are summarized here. They measure the efficiencies for muons with $p_{\mathrm{T}}$ above 10 $\mathrm{GeV} / \mathrm{c}$ in dilepton $\mathrm{Z}$ boson events using a data driven tag and probe approach. The tag and probe method is a common method in particle physics to obtain efficiencies from data. Events are selected which contain a high quality "tag" physics object as well as a lower quality "probe" object. The efficiency of the probe to pass the tag cuts is then measured. In this case they measure the single muon efficiency to be in the range of $95-97 \%$ depending upon muon $p_{\mathrm{T}}$. This agrees with the efficiency seen in the simulated signal datasets, and a trigger efficiency of (96 \pm 2$) \%$ is applied.

The efficiency to select two isolated muons with $p_{T}>10,20 \mathrm{GeV} / \mathrm{c}$ as described above is given in Table 3.5 .

In the region between the heavy Majorana neutrino masses of 70 to $100 \mathrm{GeV} / \mathrm{c}^{2}$ the efficiency of finding two muons passing the selection cuts drops considerably. This is due to the nature of the production process, where the heavy neutrino is either producing or being produced from an on shell $W$ boson. When the neutrino mass is very near to the $W$ boson mass the muon momentum drops below the cut thresholds and many events do not pass the selection.

The overall efficiency to select signal events passing the muon and additional selection cuts is shown in Table 3.6.

A visualization of a typical event in data passing these selection cuts is shown in Figures 3.4, 3.5, 3.6, and 3.7. 
Table 3.5: Muon selection and individual cut efficiencies and statistical errors for events passing each selection criteria.

\begin{tabular}{lccccc}
\hline \hline Generated & Total & & \% Events Accepted & \\
$m_{N}$ & Events & Track Quality & $\eta$ and $p_{\mathrm{T}}$ & Isolation & All \\
\hline $50 \mathrm{GeV} / \mathrm{c}^{2}$ & 49997 & $50.2 \pm 0.3$ & $25.8 \pm 0.2$ & $44.5 \pm 0.3$ & $16.8 \pm 0.2$ \\
$70 \mathrm{GeV} / \mathrm{c}^{2}$ & 49999 & $50.1 \pm 0.3$ & $8.90 \pm 0.1$ & $39.5 \pm 0.3$ & $5.2 \pm 0.1$ \\
$75 \mathrm{GeV} / \mathrm{c}^{2}$ & 49700 & $44.4 \pm 0.3$ & $7.84 \pm 0.1$ & $35.8 \pm 0.3$ & $4.7 \pm 0.1$ \\
$80 \mathrm{GeV} / \mathrm{c}^{2}$ & 49994 & $45.2 \pm 0.3$ & $13.1 \pm 0.2$ & $36.1 \pm 0.3$ & $6.0 \pm 0.2$ \\
$85 \mathrm{GeV} / \mathrm{c}^{2}$ & 49997 & $48.1 \pm 0.3$ & $14.1 \pm 0.2$ & $37.5 \pm 0.3$ & $7.5 \pm 0.1$ \\
$90 \mathrm{GeV} / \mathrm{c}^{2}$ & 48192 & $57.1 \pm 0.4$ & $20.8 \pm 0.2$ & $42.8 \pm 0.3$ & $10.3 \pm 0.2$ \\
$95 \mathrm{GeV} / \mathrm{c}^{2}$ & 49993 & $60.6 \pm 0.4$ & $33.0 \pm 0.3$ & $46.8 \pm 0.3$ & $18.2 \pm 0.2$ \\
$100 \mathrm{GeV} / \mathrm{c}^{2}$ & 49994 & $58.3 \pm 0.3$ & $42.1 \pm 0.3$ & $38.6 \pm 0.3$ & $25.1 \pm 0.2$ \\
$105 \mathrm{GeV} / \mathrm{c}^{2}$ & 49995 & $63.9 \pm 0.4$ & $49.9 \pm 0.3$ & $53.4 \pm 0.3$ & $30.8 \pm 0.3$ \\
$110 \mathrm{GeV} / \mathrm{c}^{2}$ & 49694 & $65.4 \pm 0.4$ & $56.1 \pm 0.3$ & $56.1 \pm 0.3$ & $36.2 \pm 0.3$ \\
$130 \mathrm{GeV} / \mathrm{c}^{2}$ & 48197 & $69.5 \pm 0.4$ & $66.8 \pm 0.3$ & $63.2 \pm 0.4$ & $46.7 \pm 0.3$ \\
$150 \mathrm{GeV} / \mathrm{c}^{2}$ & 49997 & $72.5 \pm 0.4$ & $71.8 \pm 0.4$ & $67.1 \pm 0.4$ & $51.4 \pm 0.3$ \\
$170 \mathrm{GeV} / \mathrm{c}^{2}$ & 48196 & $75.4 \pm 0.4$ & $75.7 \pm 0.4$ & $69.7 \pm 0.4$ & $54.7 \pm 0.3$ \\
$190 \mathrm{GeV} / \mathrm{c}^{2}$ & 49999 & $77.3 \pm 0.4$ & $78.4 \pm 0.4$ & $71.4 \pm 0.4$ & $56.7 \pm 0.3$ \\
$210 \mathrm{GeV} / \mathrm{c}^{2}$ & 47300 & $79.2 \pm 0.4$ & $81.0 \pm 0.4$ & $73.3 \pm 0.4$ & $58.4 \pm 0.4$ \\
\hline
\end{tabular}


Table 3.6: Event selection efficiencies for the selection. Errors are statistical only.

\begin{tabular}{lccccc}
\hline \hline Generated & Total & \multicolumn{3}{c}{ \% Events Accepted } & \# of Events \\
$m_{N}$ & Events & All Muon & Jets & All Cuts & for $4.96 \mathrm{fb}^{-1}$ \\
\hline $50 \mathrm{GeV} / \mathrm{c}^{2}$ & 49997 & $16.8 \pm 0.2$ & $6.20 \pm 0.1$ & $0.432 \pm 0.026$ & $18340 \pm 1104$ \\
$70 \mathrm{GeV} / \mathrm{c}^{2}$ & 49999 & $5.15 \pm 0.1$ & $9.80 \pm 0.1$ & $0.323 \pm 0.022$ & $2709 \pm 185$ \\
$75 \mathrm{GeV} / \mathrm{c}^{2}$ & 49700 & $4.71 \pm 0.2$ & $12.6 \pm 0.2$ & $0.429 \pm 0.027$ & $1360 \pm 85.6$ \\
$80 \mathrm{GeV} / \mathrm{c}^{2}$ & 49994 & $6.02 \pm 0.2$ & $20.1 \pm 0.2$ & $1.07 \pm 0.050$ & $798 \pm 37.3$ \\
$85 \mathrm{GeV} / \mathrm{c}^{2}$ & 49997 & $7.46 \pm 0.1$ & $30.7 \pm 0.3$ & $1.20 \pm 0.046$ & $483 \pm 18.6$ \\
$90 \mathrm{GeV} / \mathrm{c}^{2}$ & 48192 & $10.3 \pm 0.2$ & $34.7 \pm 0.3$ & $2.60 \pm 0.071$ & $714 \pm 19.5$ \\
$95 \mathrm{GeV} / \mathrm{c}^{2}$ & 49993 & $18.2 \pm 0.2$ & $36.9 \pm 0.3$ & $5.40 \pm 0.10$ & $1036 \pm 19.2$ \\
$100 \mathrm{GeV} / \mathrm{c}^{2}$ & 49994 & $25.1 \pm 0.2$ & $39.1 \pm 0.3$ & $7.87 \pm 0.12$ & $1074 \pm 16.4$ \\
$105 \mathrm{GeV} / \mathrm{c}^{2}$ & 49995 & $30.8 \pm 0.3$ & $40.8 \pm 0.3$ & $10.0 \pm 0.14$ & $1013 \pm 14.2$ \\
$110 \mathrm{GeV} / \mathrm{c}^{2}$ & 49694 & $36.2 \pm 0.3$ & $42.4 \pm 0.3$ & $11.9 \pm 0.15$ & $926 \pm 11.7$ \\
$130 \mathrm{GeV} / \mathrm{c}^{2}$ & 48197 & $46.7 \pm 0.3$ & $46.9 \pm 0.3$ & $17.0 \pm 0.18$ & $576 \pm 6.09$ \\
$150 \mathrm{GeV} / \mathrm{c}^{2}$ & 49997 & $51.4 \pm 0.3$ & $51.3 \pm 0.3$ & $20.7 \pm 0.19$ & $359 \pm 3.03$ \\
$170 \mathrm{GeV} / \mathrm{c}^{2}$ & 48196 & $54.7 \pm 0.3$ & $55.2 \pm 0.3$ & $23.3 \pm 0.21$ & $233 \pm 2.10$ \\
$190 \mathrm{GeV} / \mathrm{c}^{2}$ & 49999 & $56.7 \pm 0.3$ & $59.2 \pm 0.3$ & $26.3 \pm 0.22$ & $163 \pm 1.36$ \\
$210 \mathrm{GeV} / \mathrm{c}^{2}$ & 47300 & $58.4 \pm 0.4$ & $62.2 \pm 0.4$ & $28.8 \pm 0.24$ & $117 \pm 0.98$ \\
\hline
\end{tabular}




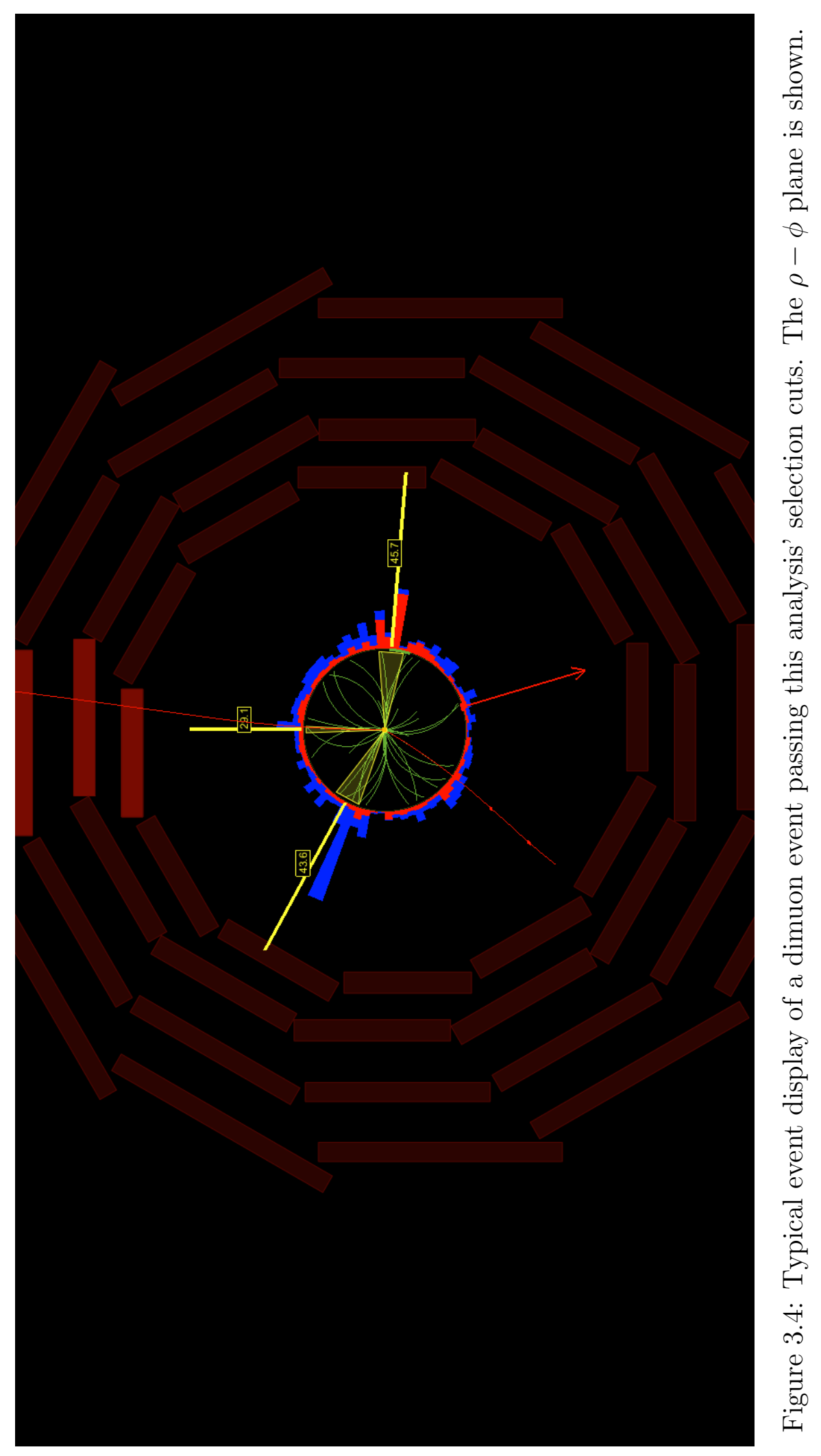




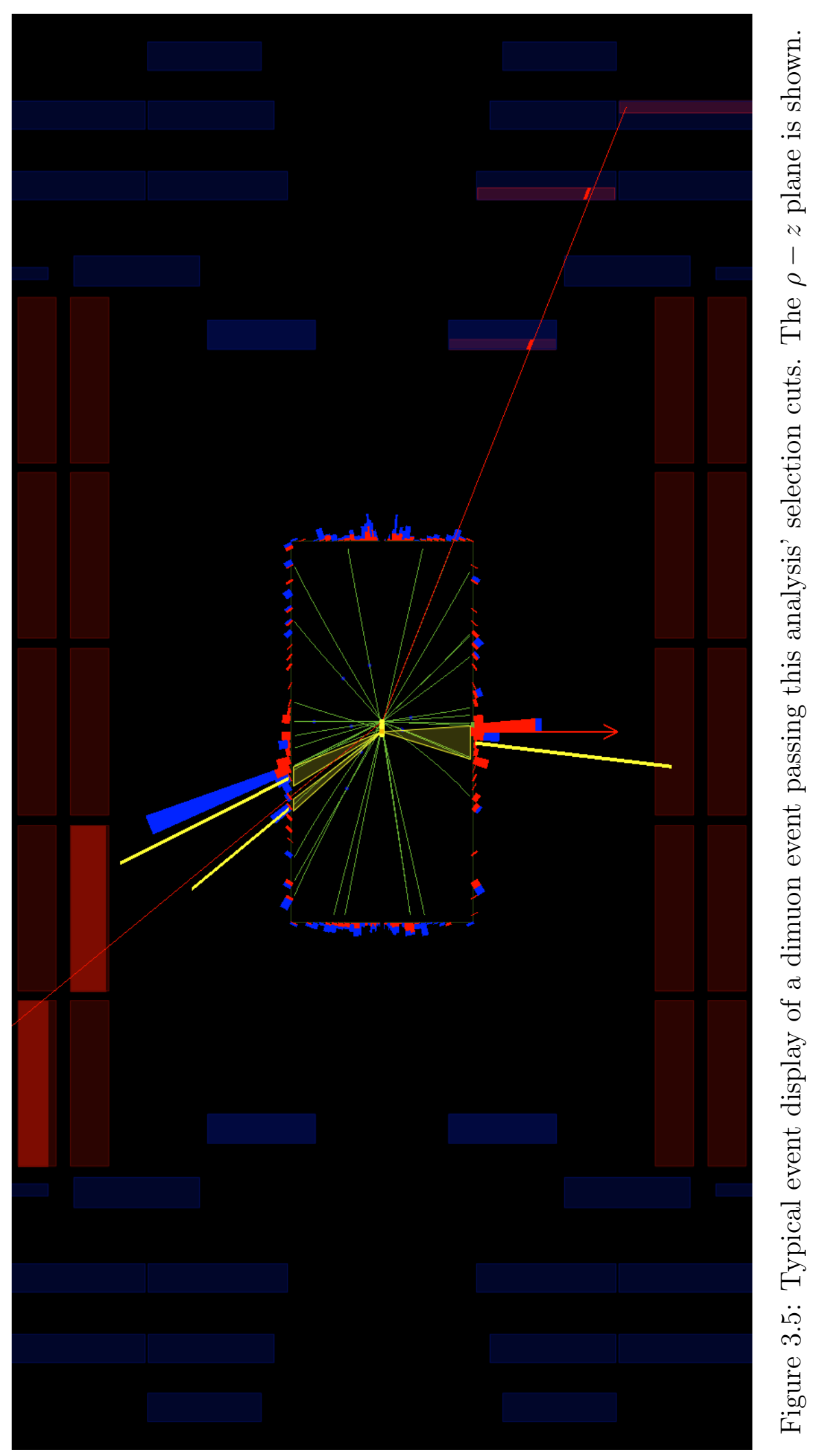




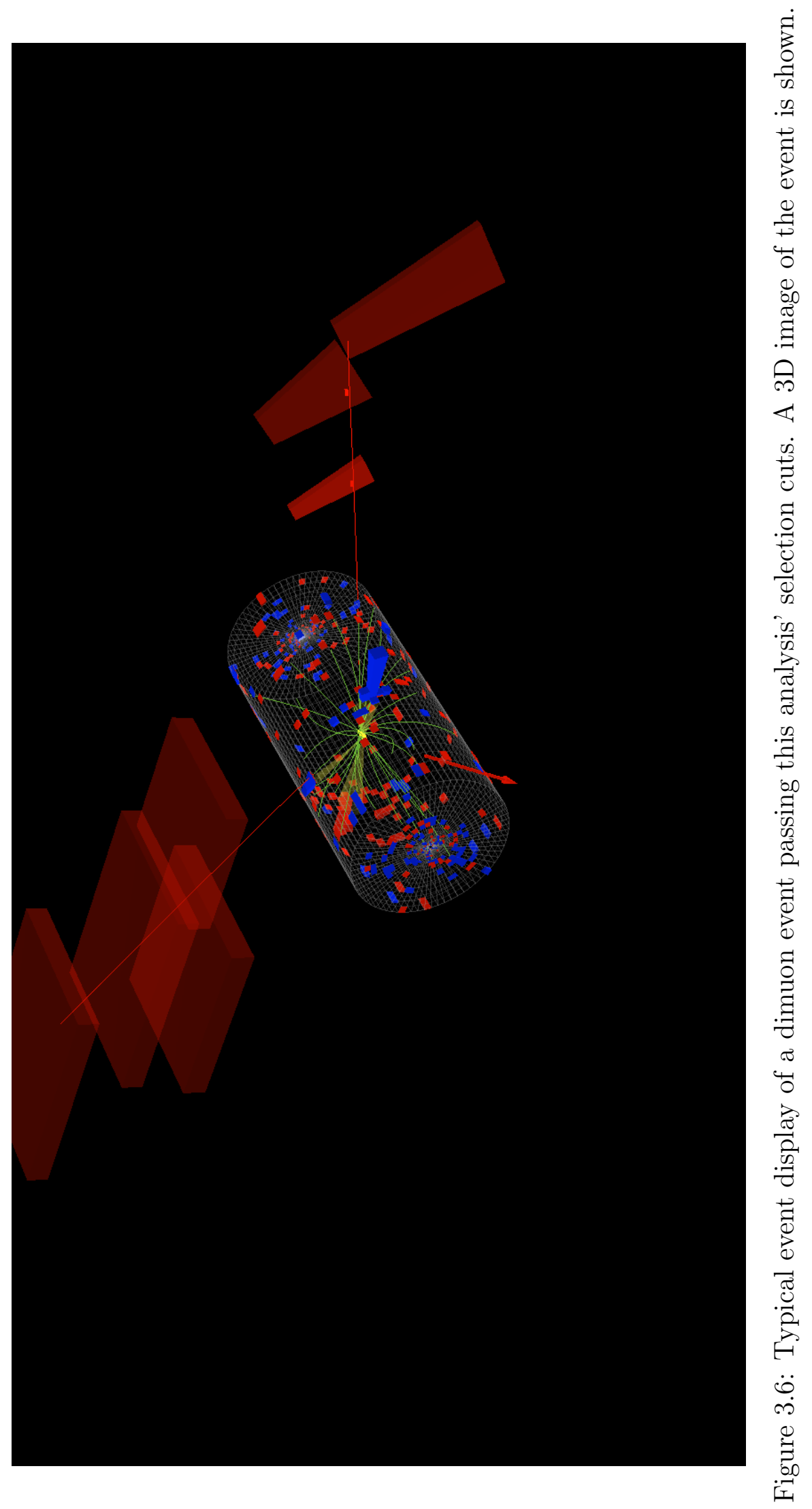





\subsubsection{Data-Monte Carlo Scale Factor}

The accuracy of the simulation of the signal events can be examined via a "tag and probe" method on $Z$ boson decaying to dilepton events. This method which has been used successfully in previous CMS analyses $[50,51,52,53]$ has been also performed for this analysis.

Single muon triggered data is used requiring that the tag muon pass the full muon selection criteria, while the other muon (the probe muon) must pass looser cuts which are in this case:

- $p_{\mathrm{T}}>5 \mathrm{GeV} / \mathrm{c}$

- $|\eta|<2.4$

- be both a tracker and global muon type

A detailed description of the method used to extract the efficiencies is discussed in References $[50,53]$. In summary, the efficiency is $\epsilon=T P /(T P+T F)$ where $T P$ is the number of probes that pass the selection cuts being studied (isolation or track quality) and $T F$ is the number that fail. These numbers are found from fitting the tag-probe muon pair mass distributions with a signal model taken from simulation, see Figure 3.8 for an example, and an exponential to describe the background. Figure 3.9 provides an example of the resulting fit to data. In the kinematic range of the muons produced by the heavy neutrino signal the muons are well modeled by the simulation as seen in Table 3.7. A systematic error of $1 \%$ on the muon identification and isolation is estimated by comparing these results to the same calculation made by counting the number of dimuon tag probe pairs passing the selection criteria whose masses lies within the same mass window used in the signal analysis cuts to veto on $Z$ bosons. The background contribution can be estimated by looking at the 
same sign pairs in the same mass window. There is an additional systematic uncertainty on the efficiency of finding isolated muons with low momentum; however, the effect is too small to be seen in this analysis' results.

Table 3.7: Muon identification and isolation efficiencies for both simulation and data measured with the tag and probe method.

\begin{tabular}{ccccc}
\hline Criteria & $p_{\mathrm{T}}$ range $(\mathrm{GeV} / \mathrm{c})$ & Data & $\mathrm{MC}$ & Data/MC \\
\hline \hline Iso & $10-20$ & $0.736 \pm 0.003$ & $0.753 \pm 0.006$ & $0.978 \pm 0.009$ \\
& $>20$ & $0.893 \pm 0.001$ & $0.903 \pm 0.001$ & $0.990 \pm 0.002$ \\
ID & $10-20$ & $0.987 \pm 0.001$ & $0.989 \pm 0.001$ & $0.998 \pm 0.002$ \\
& $>20$ & $0.997 \pm 0.004$ & $0.990 \pm 0.007$ & $0.993 \pm 0.008$ \\
Total & $10-20$ & & & $0.975 \pm 0.009$ \\
& $>20$ & & & $0.983 \pm 0.008$ \\
\hline
\end{tabular}

\subsubsection{Backgrounds}

The main backgrounds in this analysis originate from events in which either one muon is fake (e.g. $t \bar{t}$ and $W+$ jets) or both muons are fake (e.g. QCD multijet events). In the first case one muon originates from a $W$ decay, while the second originates from a jet (e.g. a jet from a $b$ quark decay) and is typically less isolated. In the second case both muons originate from jets. A data driven "tight to loose" method is used to estimate these backgrounds. It is expected is that since the signal has region no $E_{\mathrm{T}}^{\text {miss }}$ the fake background will be dominated by QCD events. This is because both $t \overline{\mathrm{t}}$ and $\mathrm{W}+$ jets produce $E_{\mathrm{T}}^{\mathrm{miss}}$ and while QCD does not often produce fakes it has a very large cross section in the low $E_{\mathrm{T}}^{\mathrm{miss}}$ region. While double fakes may come from any type of event, it is expected that most will come from QCD processes, thus we expect there should be more double fakes than single fakes. In 


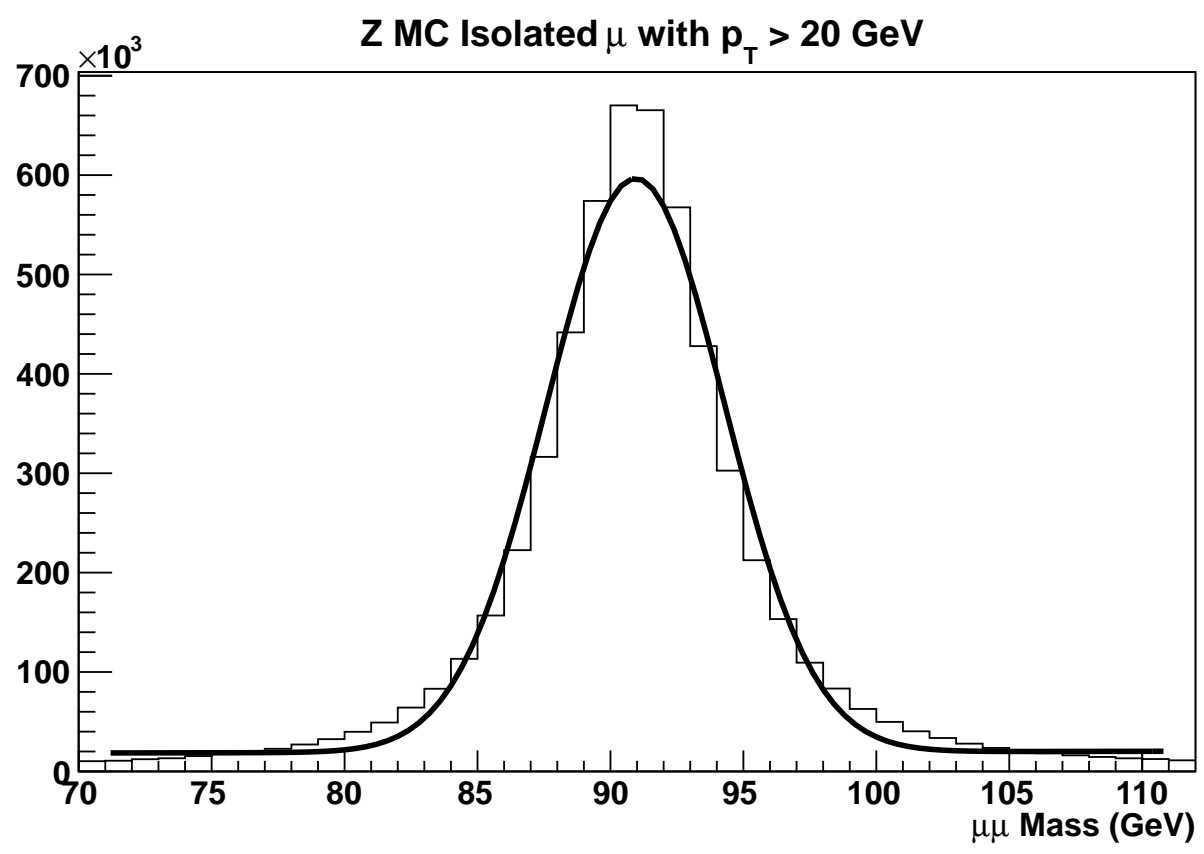

Figure 3.8: This shows an example fit to a dimuon mass spectrum from the Z+jets Monte Carlo listed above where probe muon was required to pass the full isolation cuts and have a $p_{\mathrm{T}}$ above $20 \mathrm{GeV}$.

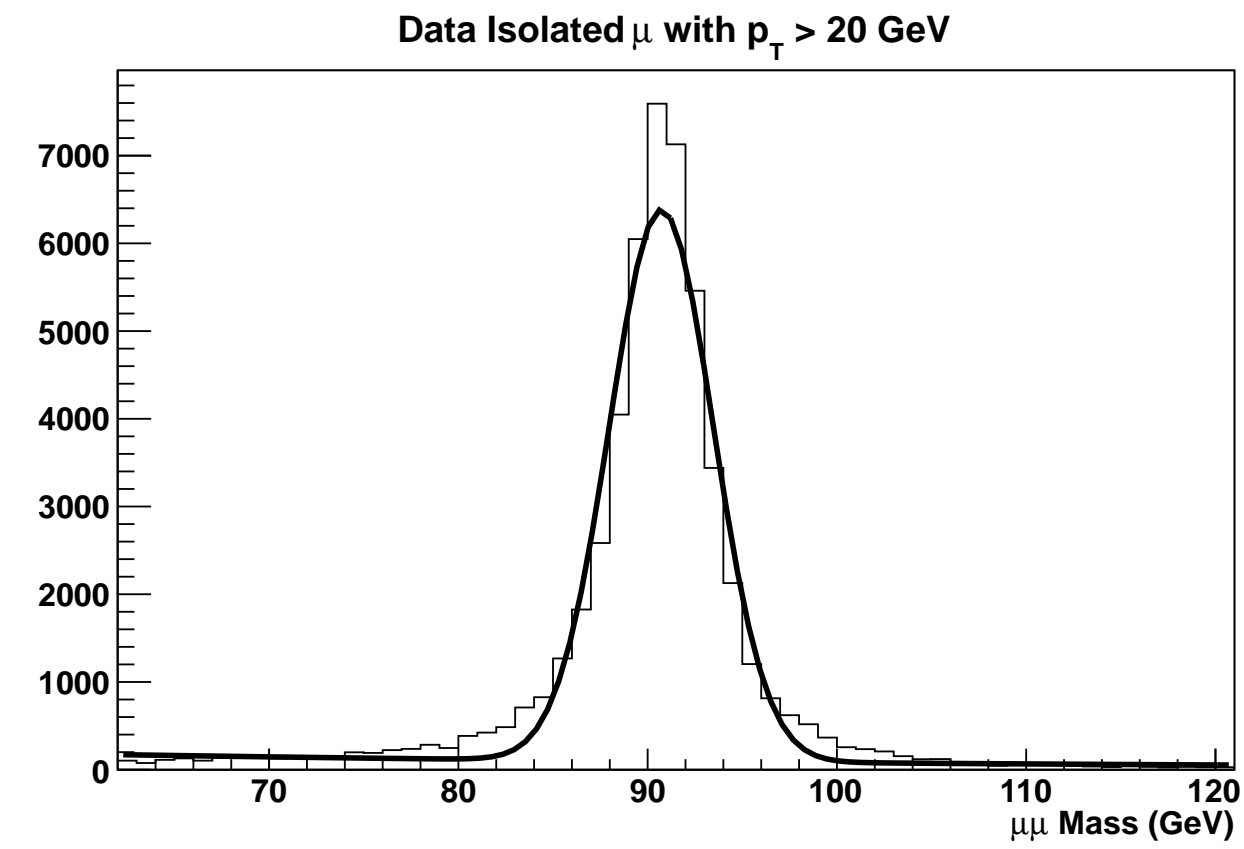

Figure 3.9: This shows an example fit to a dimuon mass spectrum from the data using the fit from Figure 3.8; where again the probe muon was required to pass the full isolation cuts and have a $p_{\mathrm{T}}$ above $20 \mathrm{GeV}$. 
practice the number of single and double fakes also is affected by the way the data driven method is performed. Due to this the more important result is the over all prediction which is stable under different "tight to loose" definitions.

The sources of real same-sign dimuon events in the standard model are also taken into account. They are much smaller than the fake background and originate mainly from diboson and $t \bar{t} W$ production. They are estimated using Monte Carlo samples.

The background from muon charge mismeasurement was considered and found to be negligible.

The "tight to loose" method is used to estimate the background due to fake muons. This method is described in Reference. [54] and has been used in the CMS same-sign dilepton SUSY search $[51,52]$ as well as the Higgs search in the $H \rightarrow W^{+} W^{-} \rightarrow \ell^{+} \nu \ell^{-} \bar{\nu}$ channel [50]. Here is a basic description of the method; see Reference [54] for a more detailed description.

An independent data sample dominated by QCD jets is used to calculate a fake rate (also known as a tight-to-loose ratio or $\mathrm{T} / \mathrm{L}$ ratio), defined as the fraction of muon candidates passing loose cuts that also pass the tight cuts used in the analysis to select the final event sample. The muon candidates passing the loose cuts are referred to as "fakeable objects". The fake rate is calculated as a function of fakeable object transverse momentum and pseudorapidity. The fake rate is then used as an event weight to calculate the background using a sample events which pass all the signal selection cuts, except that one or both muons fail the tight selection. This sample is referred to as the "orthogonal" sample.

The application of the fake rate to the orthogonal sample is obtained by weighting the counts of the number of events in which one lepton passes the tight cuts and the other muon fails the tight selection but passes the loose selection $\left(N_{n \bar{n}}\right)$ 
and the number of events in which both leptons fail the tight selection but pass the loose cuts $\left(N_{\bar{n} \bar{n}}\right)$. The total contribution to the signal sample (i.e. the number of events in which both muons pass the tight selection, $\left.N_{n n}\right)$, is given by

$$
\begin{aligned}
N_{n n} & =\sum_{i, j} \frac{F R_{i} F R_{j}}{\left(1-F R_{i}\right)\left(1-F R_{j}\right)} N_{\bar{n} \bar{n}}^{i j} \\
& +\sum_{i, j} \frac{F R_{i}}{\left(1-F R_{i}\right)}\left[N_{n \bar{n}}^{i j}-\frac{F R_{i}\left(1-F R_{j}\right)+F R_{j}\left(1-F R_{i}\right)}{\left(1-F R_{i}\right)\left(1-F R_{j}\right)} N_{\bar{n} \bar{n}}^{i j}\right]
\end{aligned}
$$

where $F R$ is the fake rate and the indices $i, j$ label the two muons in the event. Note that in the square parentheses, the second term is a correction to $N_{n \bar{n}}^{i j}$ due to $\bar{n} \bar{n}$ events that can be also be $n \bar{n}$.

In data fakeable objects are defined by relaxing the muon isolation requirement from RelIso $<0.1$ (used in the signal event selection) to RelIso $<0.8$. The independent control sample used to obtain the fake rate is selected from the single muon triggers primary dataset mentioned in Section 3.5.1. A logical OR of the same triggers used in the tag and probe study was used to produce the data sample.

- HLT_Mu8

- HLT_Mu8_Jet40

- $\mathrm{HLT} \_\mathrm{Mu} 15$

Events must contain one muon with $p_{T}>10 \mathrm{GeV}$ passing the loose requirements and one jet on the opposite side to the muon $\left(\Delta R_{\mu j}>1.0\right)$ satisfying all the same selection cuts used in the analysis except the $p_{T}$ requirement, which is set at $p_{T}>40 \mathrm{GeV}$. This cut is chosen to match as closely as possible the expected $p_{T}$ distribution of jets in the orthogonal data sample. In order to reject $W$ events, the cuts $E_{\mathrm{T}}^{\mathrm{miss}}<20 \mathrm{GeV}$ and $M_{T}<20 \mathrm{GeV}$ are imposed. To suppress $Z$ events, dimuon events with an invariant mass $m_{\mu \mu}$ within $20 \mathrm{GeV}$ of the $Z$ boson mass are rejected. The fake rate is corrected for electroweak contamination by subtracting 
the Monte Carlo contributions due to electroweak processes from the event samples used to calculate the fake rates, normalized to the trigger luminosities of the three triggers used. The fake rate is obtained in bins of $\eta$ and $P_{t}$.

The fake rate method may be tested on Monte Carlo samples in which the true background is known. The misidentification probabilities are obtained from multijet events and are used to estimate the misidentified lepton backgrounds in t $\bar{t}, \mathrm{~W}+$ jets, and multijet events. A full description of this method and its results may be found in Reference [55]. The predicted backgrounds for misidentified leptons agree with the actual backgrounds to within 35\%. Based upon this and an observation of the variability in the fake rate when the fakeable object definition is changed the systematic error on this prediction can be taken as $35 \%$.

A cross check can also be performed on the background estimation method in data by using a signal-free control region in which all selection cuts are applied except that the missing transverse energy is required to be $E_{\mathrm{T}}^{\mathrm{miss}}>50 \mathrm{GeV}$. The results from this cross check are shown in Table 3.8. The predicted total background of $55.1 \pm 2.0$ (stat.) \pm 15.8 (syst.) events is within $15 \%$ of the 48 events observed. This is well within the $35 \%$ systematic uncertainty on the background prediction, showing that the fake background prediction method performs as expected.

With a good understanding of the expected background the observed data can now be compared to the expectation, where an excess could indicate new physics. There are 65 events observed in data, and the final background estimates are given in Table 3.9. The total background estimate is $70.4 \pm 4.2$ (stat.) \pm 22.1 (syst.) events, with the dominant contribution arising from the fake muon background $(63.1 \pm 4.2$ (stat.) \pm 22.1 (syst.) events). No excess is observed so an exclusion on the production of a heavy Majorana neutrino may be done. 
Table 3.8: Observed event yields and estimated backgrounds with statistical and systematic uncertainties are shown for the $E_{\mathrm{T}}^{\text {miss }}>50 \mathrm{GeV}$ control region.

\begin{tabular}{ll}
\hline \hline Source & Events \\
\hline Monte Carlo: & \\
$W Z$ & $3.16 \pm 0.30$ (stat.) \pm 0.13 (syst.) \\
$Z Z$ & $0.19 \pm 0.04$ (stat.) \pm 0.01 (syst.) \\
$W \gamma$ & $0.31 \pm 0.17$ (stat.) \pm 0.03 (syst.) \\
$t \bar{t} W$ & $3.19 \pm 0.10$ (stat.) \pm 1.60 (syst.) \\
$W^{+} W^{+}$ & $2.57 \pm 0.10$ (stat.) \pm 1.29 (syst.) \\
$W^{-} W^{-}$ & $0.84 \pm 0.04$ (stat.) \pm 0.42 (syst.) \\
double parton $W^{ \pm} W^{ \pm}$ & $0.08 \pm 0.02$ (stat.) \pm 0.04 (syst.) \\
Total Monte Carlo & $10.34 \pm 0.37$ (stat.) \pm 2.10 (syst.) \\
\hline Data driven background estimate: & \\
Total fake background & $44.74 \pm 2.00$ (stat.) \pm 15.66 (syst.) \\
\hline Total background & $55.08 \pm 2.03$ (stat.) \pm 15.80 (syst.) \\
\hline Observed in data (4.98 fb ${ }^{-1}$ ) & 48 \\
\hline
\end{tabular}


Table 3.9: Observed event yields and estimated backgrounds with statistical and systematic uncertainties for the analysis, with $4.98 \mathrm{fb}^{-1}$, for the signal region $\left(E_{\mathrm{T}}^{\mathrm{miss}}<50 \mathrm{GeV}\right)$.

\begin{tabular}{ll}
\hline \hline Source & Events \\
\hline Monte Carlo: & \\
$W Z$ & $3.22 \pm 0.3$ (stat.) \pm 0.13 (syst.) \\
$Z Z$ & $1.02 \pm 0.10$ (stat.) \pm 0.04 (syst.) \\
$W \gamma$ & $0.75 \pm 0.27$ (stat.) \pm 0.07 (syst.) \\
$t \bar{t} W$ & $1.06 \pm 0.05$ (stat.) \pm 0.53 (syst.) \\
$W^{+} W^{+}$ & $0.76 \pm 0.06$ (stat.) \pm 0.38 (syst.) \\
$W^{-} W^{-}$ & $0.45 \pm 0.03$ (stat.) \pm 0.23 (syst.) \\
double parton $W^{ \pm} W^{ \pm}$ & $0.07 \pm 0.02$ (stat.) \pm 0.04 (syst.) \\
Total Monte Carlo & $7.33 \pm 0.42$ (stat.) \pm 0.71 (syst.) \\
\hline Data-driven background estimate: & \\
Fake muon background & $63.09 \pm 4.20$ (stat.) \pm 22.08 (syst.) \\
\hline Total background & $70.42 \pm 4.22$ (stat.) \pm 22.09 (syst.) \\
\hline Observed in data (4.98 fb ${ }^{-1}$ ) & 65 \\
\hline
\end{tabular}




\subsubsection{Error Analysis}

As this is a counting experiment the dominant sources of systematic uncertainties are those associated with the understanding of the signal model, efficiencies, and the background prediction. Uncertainties are taken into account from the following sources:

- Integrated Luminosity: The systematic uncertainty on the integrated luminosity is $2.2 \%$.

- Parton Distribution Functions: The ALPGEN signal Monte Carlo parton distribution function uncertainty is estimated using the method in Reference [56]. The resulting uncertainty is $5 \%$ on the signal yield and $1 \%$ on the signal acceptance.

- $Q^{2}$ Scale: The event $Q^{2}$ scale in the ALPGEn signal Monte Carlo generator is varied from $4 Q^{2}$ to $Q^{2} / 4$. The resulting uncertainty is $1 \%$.

- Muon Trigger and Selection: Based upon the results of the tag and probe study and the trigger studies in Reference [50] a $2 \%$ uncertainties is assigned for the trigger efficiency and $2 \%$ for the muon selection efficiency.

- Jet Energy Scale: The jet energy may be scaled by the official energy uncertainty [57] and then the resulting effect on the signal efficiency is observed. The resulting systematic uncertainty is between $3.3-14.2 \%$ and depends on the overall importance of the jet $p_{\mathrm{T}}$ cut. In the lower Majorana neutrino mass range it has a larger effect (up to $14.2 \%$ at $m_{N}=50 \mathrm{GeV} / \mathrm{c}^{2}$ ), while at $210 \mathrm{GeV} / \mathrm{c}^{2}$ is decreases to $3.3 \%$.

- Jet Energy Resolution: The jet energy uncertainty is scaled by an additional 
$10 \%$ and again observed for any additional effect this has on the signal efficiency. An additional systematic of $0.2 \%-1 \%$, depending on Majorana neutrino mass, is observed.

- Pile Up Model: As recommended by the in Reference [58] the number of interactions is smeared by \pm 0.6 for the purpose of calculating the uncertainty due to pile up modeling in the signal simulations. This results in a systematic uncertainty of about $1 \%$.

- Background Estimate: The uncertainty on the background estimate is $35 \%$ and is discussed briefly in Section 3.5.4 and in greater detail in Reference [55].

The uncertainties in the muon transverse momentum energy scale were found to be between $1.3 \%-6 \%$ depending on muon pseudorapidity [59]. The effect of this error on the overall signal efficiency is negligible. A summary of the systematic uncertainties is given in Table 3.10.

\subsubsection{Setting Exclusion Limits}

There now must be an attempt to understand what information has been gained from the results of this analysis. When there is not an observed excess in the number of events above the predicted background, it standard to quantify a region of phase space considered excluded according to a commonly accepted level of statistical confidence. This is a description of a standard way to set this exclusion.

This type of analysis's results have been formulated within the framework of classical statistical hypothesis testing. For this test one defines two hypotheses, the null and alternate (or signal) hypotheses. Reference [60] summarizes it thusly

The null hypothesis is the background hypothesis 'b', i.e. the data can be understood with existing physics explanations. The alternative hypothesis, which is favored when the null hypothesis has been rejected to 
Table 3.10: Summary of systematic uncertainties across the mass range studied.

\begin{tabular}{lccc}
\hline \hline & \multicolumn{2}{c}{ Majorana Neutrino } \\
& \multicolumn{2}{c}{ Mass $\left(\mathrm{GeV} / \mathrm{c}^{2}\right)$} \\
Source & 70 & 130 & 190 \\
\hline Mass-dependent uncertainties: & & & \\
Jet Energy Scale & $12 \%$ & $7 \%$ & $4 \%$ \\
Jet Energy Resolution & $0.68 \%$ & $0.23 \%$ & $0.23 \%$ \\
Pile Up Model & $1.7 \%$ & $0.48 \%$ & $0.16 \%$ \\
Mass-independent uncertainties: & & & \\
Integrated Luminosity & $2.2 \%$ & $2.2 \%$ & $2.2 \%$ \\
Parton distribution Functions & $5 \%$ & $5 \%$ & $5 \%$ \\
Event Q ${ }^{2}$ scale & $1 \%$ & $1 \%$ & $1 \%$ \\
Muon Trigger & $2 \%$ & $2 \%$ & $2 \%$ \\
Muon Selection & $2 \%$ & $2 \%$ & $2 \%$ \\
Background Prediction & $35 \%$ & $35 \%$ & $35 \%$ \\
\hline
\end{tabular}


a sufficient degree, is that we need new physics to understand the data.

In this analysis there is background present even after all selection criteria are applied, so the two hypotheses are labeled $B$, for the background-only (or null) hypothesis, and $S+B$ for signal plus background which is the alternate hypothesis. We then follow the procedure outlined in these steps:

1. Identify the observables. This analysis uses a simple counting experiment, and the final observable has been defined as the number of events passing all selection criteria, $N_{\text {obs }}$.

2. Define a test statistic $Q$ that is a function of the defined observables and other parameters of the model. The definition of $\mathrm{Q}$ is discussed below. Other parameters of the model, also known as nuisance parameters, are defined here as the final yields of the expected background events from different processes, their total fractional uncertainties, the uncertainty in the number of signal $(S)$ events passing the selection criteria, and the uncertainty in the integrated luminosity of the data used in this analysis.

3. Define the rules for exclusion and discovery, which are the ranges of values of the test statistic for which the signal is considered to be discovered or excluded. A confidence limit is defined which is a lower limit on $\left|V_{\mu N}\right|^{2}$ as a function of $m_{N}$ where the signal is said to be excluded to a confidence level (CL) of $95 \%$.

The test statistic is usually designed to increase monotonically, so that small (or large) values signify increasingly signal-like (or background-like) data. This is the procedure that this analysis adopts. The confidence in a hypothesis $H$ then becomes the probability that, given the hypothesis, the test statistic $Q$ is less than or equal to the value observed in the experiment, $Q_{o b s}$, or 


$$
C L_{H}=P_{H}\left(Q \leq Q_{o b s}\right)=\int_{-\infty}^{Q_{o b s}} \frac{d P_{H}}{d Q} d Q
$$

where $d P_{H} / d Q$ is the probability density function, or p.d.f., of the test statistic for hypothesis $H$. For the sense of a $Q$ as is adopted above, when $H=S+B$, a high confidence in $H$ is signified by high values (close to 1 ) of $C L_{H}=C L_{s+b}$; but for $H=B$, high confidence in $H$ is signified by low values (close to 0 ) of $C L_{H}=C L_{b}$.

It is typical to speak of the probability $P$ of a possible outcome that $X$ number of events would be observed given a certain model $M$ with a set of parameters $\Theta$, which is notated $P(X \mid \Theta)$. However, in this case the interest in in the reverse, the data that has been observed is the given, and the interest is in saying something definitive about the theory. This requires the introduction of the concept of a likelihood. Likelihood is very similar to a probability but instead of the above case where the probability discusses an observational outcome given a model the likelihood discusses what is known of a model given an observational outcome. Thus the likelihood that the model $M(\Theta)$ is true for the observed data $X$, notated $L(\Theta \mid X)$, is what needs to be obtained. In general $L(\Theta \mid X)$ and $P(X \mid \Theta)$ are proportional by some arbitrary constant. To eliminate this constant ratios of likelihoods are used. It is then possible to speak of whether one model is more likely than another, given the observed data.

In this case the parameter of interest is simply a number of events, so the probability $P$ is replaced with a p.d.f that is a statistical distribution describing the probability of observing $X$ events; for a simple case like this it is usually a Poisson. This analysis has two p.d.f.'s $B\left(N_{o b s}\right)$, corresponding to the hypotheses $H=B$, and the signal plus background p.d.f. $S B\left(N_{o b s}\right)$, corresponding to hypothesis $H=S+B$. It s now possible to define the test statistic $Q$ to be the ratio of likelihoods $L(\Theta \mid X)$, which given the above is simply the ratio of p.d.f.'s: 


$$
Q=\left(\frac{\prod_{i} S B\left(N_{i}\right.}{\prod_{i} B\left(N_{i}\right.}\right)
$$

Finally, it is the general process to take the natural logarithm of $Q$ to form the "log likelihood ratio." This turns the products into sums while also maintaining the sense of $Q$ described above:

$$
L L R=\log (Q)
$$

In searches for new physics if the available statistics are small and the observed events oscillate low, the background hypothesis can sometimes be called into question. In this search the observed is in fact lower than the prediction of the background hypothesis (although well within the uncertainties). In cases of this type there is a method that has been established to gain some meaning from the results of the classical "frequentist" approach of $C L_{s+b}$ that has been described above. This method, called the $C L_{s}$ method, modifies the above method by normalizing the confidence level from the null hypothesis:

$$
C L_{s}=\frac{C L_{s+b}}{C L_{b}}
$$

This does not strictly speaking result in a confidence limit, but the signal hypothesis can still be considered excluded at the confidence level $C L=95 \%$ when

$$
1-C L_{s} \leq C L ; C L_{s} \geq 0.05
$$

An analysis package RooStATs [61] coupled with a counting experiment package wrapper developed by the CMS Statistical Tools group (c195) [62] is used to automate the hypothesis testing that has been just described. RooStATs is a package addition to the ROOT data analysis software package that allows for sophisticated statistical modeling and hypothesis testing. It automates much of the 
machinery of the $C L_{s}$ method. The c195 RooStats based packaged automates the following procedure:

- Instantiate p.d.f's of the background prediction.

- Instantiate a signal p.d.f., one for each mass $\left(m_{N}\right)$ hypothesis using the Monte Carlo prediction of the number of events that remain after the full selection.

- Identify the uncertainties in the background, signal prediction, and integrated luminosity as "nuisance parameters" of the models to be varied over a number of statistical trials. A log-normal distribution is used for these nuisance parameter uncertainties.

- Invoke a hypothesis test that produces an ensemble of pseudo-experiments based on the $S B\left(N_{o b s}\right)$ and $B\left(N_{o b s}\right)$ distributions. The distribution of LLR values is used to determine the confidence levels for the signal plus background hypothesis $\left(C L_{s+b}\right)$ and for the background-only hypothesis $\left(C L_{b}\right)$.

- Calculate $C L_{s}$ from the hypothesis test results.

The software then repeats each pseudo-experiment over a range of scan points for each $m_{N}$ hypothesis. That is, the signal p.d.f. is scaled in steps over a cross section interval that is intended to provide complete coverage up to the "bare" cross sections seen in Figure 3.2 (defined as the cross section for a mixing of 1.0). This scan may be considered a "brute force" computing method to determine the contour of the Majorana neutrino mixing element squared above which the signal hypothesis can be said to be excludable at $95 \% \mathrm{CL}$, taking into account all uncertainties that have been quantified. 


\subsubsection{Results}

There is no significant excess of events in the data beyond the predicted background. Therefore, limits on the square of the Majorana neutrino mixing element based on the number of observed events, the predicted number of background events, and the predicted number of events from Majorana neutrino production are set. The resulting limits on $\left|V_{\mu N}\right|^{2}$ as a function of $m_{N}$ for $C L=0.95$ are shown in Figure 3.10. Also shown are the currently existing limits from the only other direct searches for this type of neutrino. These were done by DELPHI [36] and L3 [37]. Not shown is the $90 \%$ CL indirect limit from a global fit to electroweak precision data of $\left|V_{\mu N}\right|^{2}<0.0060$ [63]. This result provides the first direct search limits for a Majorana mass above $90 \mathrm{GeV} / \mathrm{c}^{2}$ and has been published in Physics. Letters B [55]. The CMS results provided a significant extension to the studied Majorana mass range, but were not able to improve upon the DELPHI and L3 limits. In the lower mass range, the $p_{T}$ of the final state muons and jets is often below the detectors resolution and trigger level; therefore, the efficiency of observing events in this low mass range becomes very low as can be observed in Table 3.6. This will not improve in the 2012 data where the energy and luminosity of the collisions will increase.

The $95 \% C L_{s}$ upper limit on the cross section times acceptance times efficiency $(\sigma A \epsilon)$ can also be calculated. The observed limit is $(\sigma A \epsilon)_{95}=5.39 \mathrm{fb}$ and the expected limit is $(\sigma A \epsilon)_{95}=5.26 \mathrm{fb}$.

\subsubsection{Future Prospects}

This analysis was also conducted in the dielectron channel which is covered in the publication (Reference [55]) as well. Both analyses will be repeated with the 2012 LHC collision data. This collision data, from $8 \mathrm{TeV} / \mathrm{c}^{2}$ center of mass collisions, is being produced at a higher luminosity than was present in the 2011 runs. Due 


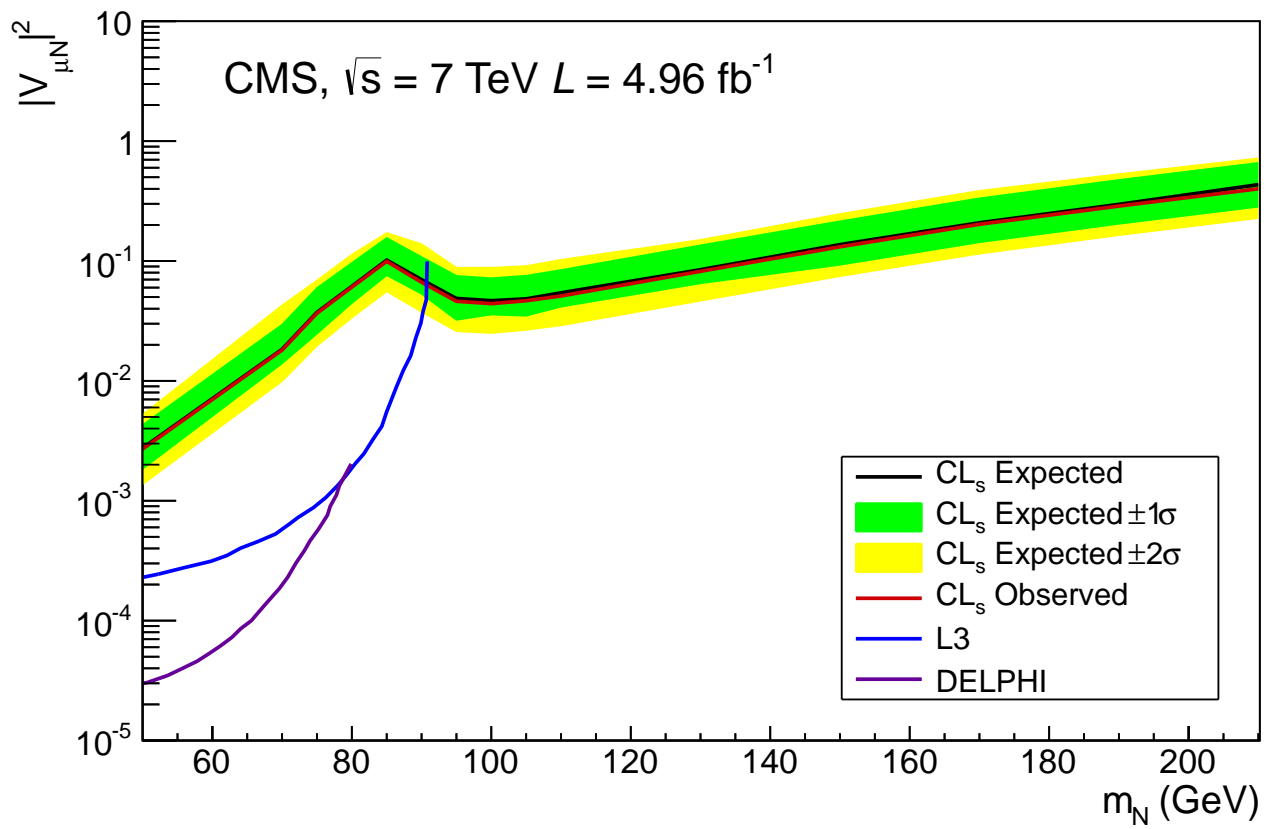

Figure 3.10: Observed exclusion region in the Majorana neutrino mixing element squared vs. mass plane obtained using the $C L_{s}$ method is above the red line; where the black line is the expected limit (with one and two sigma bands in green and yellow respectively) and also shown are the limits from DELPHI and L3. 
to this the possibilities for exclusion are much increased. Figure 3.11 shows the current exclusion results that have been discussed in this thesis as well as the same exclusion curve scaled and extrapolated to the possibilities presented by the 2012 data. There will need to be changes made to the analysis selection criteria, so this serves only as a rough idea of what may come from the 2012 data. The analysis may also be extended beyond the two channels already studied, mixed muon and electron dilepton channels are possible as is the study of trilepton processes where the final state includes a lepton and $E_{\mathrm{T}}^{\text {miss }}$ from the $\mathrm{W}$ boson decay rather than two jets.

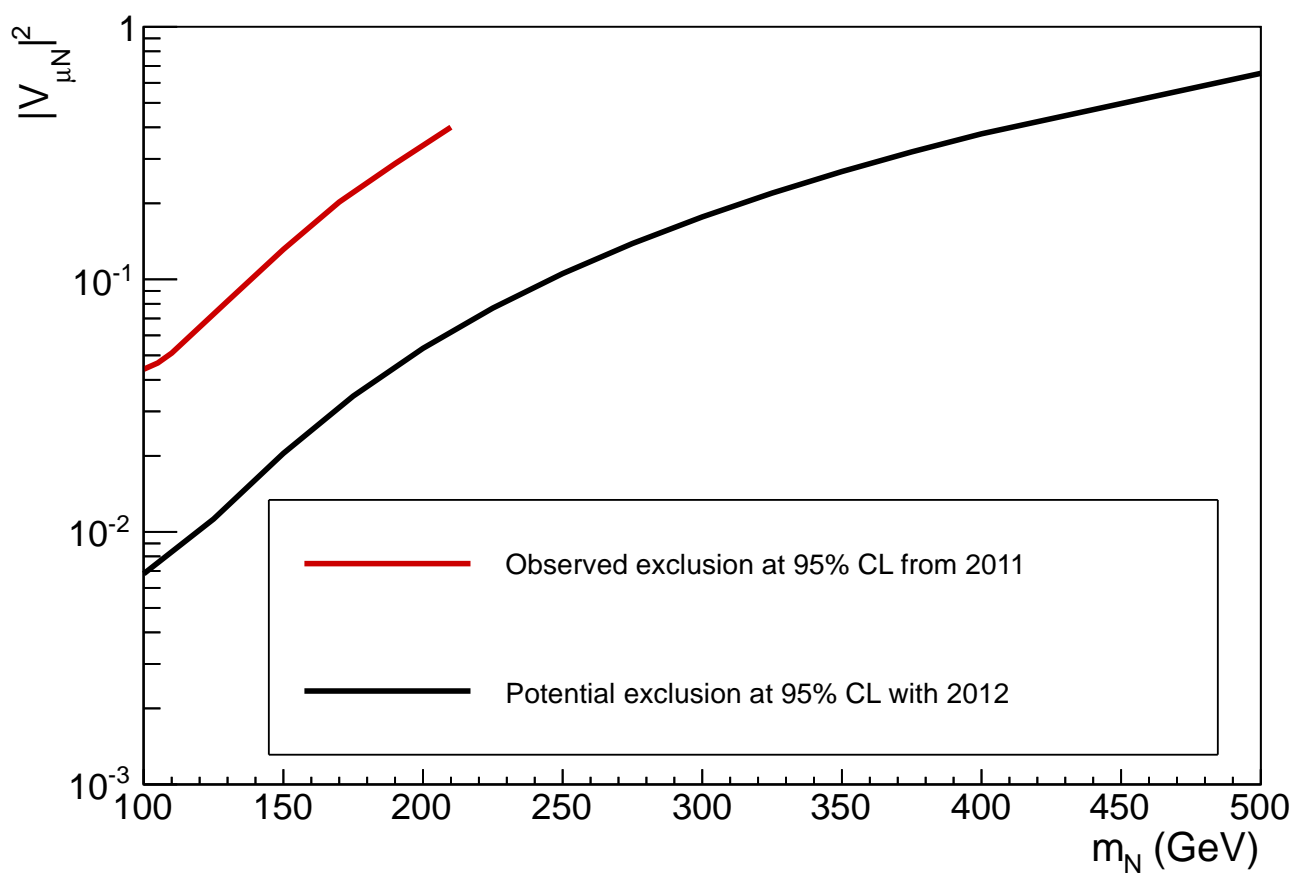

Figure 3.11: Comparison of the exclusion region in the Majorana neutrino mixing element squared vs. mass plane from the 2011 data above the red line study and the possible exclusion from the 2012 data above the black line. 


\section{CHAPTER 4 \\ QUARTZ PLATE CALORIMETER PROTOTYPE}

\subsection{Motivation and Introduction}

The LHC is designed to provide $14 \mathrm{TeV} / \mathrm{c}^{2}$ proton-proton collisions every $25 \mathrm{~ns}$; an energy level which is planned to be reached at the end of the 2013/2014 shut down period with a nominal instantaneous luminosity of $10^{34} \mathrm{~cm}^{-2} \mathrm{~s}^{-1}$. Subsequently, the integrated luminosity is planned to increase by a factor of 5 around 2017 or 2018 and finally upgrades in 2022 will increase it again for a final nominal instantaneous luminosity of $10^{35} \mathrm{~cm}^{-2} \mathrm{~s}^{-1}[24,64]$. The high luminosity runs will require substantial upgrades to some of the detector systems, such as the Hadronic Endcap (HE) calorimeters, which are positioned at both ends of the detector. These calorimeters are especially essential on jet and missing transverse energy reconstruction in the pseudorapidity region of $1.8-3$ [24].

The CMS HE calorimeters are described in Section 2.2.3. The Kuraray SCSN81 scintillators and Kuraray Y-11 wavelength shifting (WLS) fibers have been shown to be moderately radiation resistant up to $25 \mathrm{kGy}$. The simulation studies on high luminosity runs predict radiation levels up to $10 \mathrm{kGy}$ in high $\eta$ towers. Moreover, this value reaches up to $30 \mathrm{kGy}$ for the front towers where the Electromagnetic Endcap (EE) calorimeter does not shield the HE calorimeter [65, 66, 67, 68, 69].

As a solution to this radiation damage problem, it is proposed to substitute the scintillators with quartz plates $[70,71,72]$. The major advantage of such replacement is the radiation hardness of quartz material. Although there are some variations between different types, quartz shows robust performance under electron and proton irradiations $[73,74]$. On the other hand light production within quartz is through the Cherenkov process, in which the number of generated photons is inversely proportional to the wavelength, and increases in deep UV. The number of 
photons that a charge particle creates within $5 \mathrm{~mm}$ thick quartz plate is 2 orders of magnitude less than the same size plastic scintillator. Currently the University of Iowa HEP group is pursuing two different approaches to overcome this discrepancy: i) covering the surface of the quartz plates with various radiation hard, UV absorbing, wavelength shifter chemicals (such as pTp, or $\mathrm{ZnO}$ ) and readout of the signal from the edge of the plate [75]. ii) carrying the signal away from high radiation and magnetic field region by using WLS fibers. Duru et al. outlined an effective method of collecting the Cherenkov light within quartz by using WLS fibers in a bar-shape geometry [76]. However, the performance of such a calorimeter was not reported. This is a follow up to the idea of using WLS fibers, and summarizes the beam test and simulation results of the quartz plate calorimeter prototype prepared with UV absorbing WLS fibers.

\subsection{Prototype Design}

The WLS fiber type and design are crucial for improving the light collection efficiency. The Saint Gobain BCF-412 plastic WLS fibers [77], which can absorb photons down to $280 \mathrm{~nm}$, and emit at $435 \mathrm{~nm}$ are good candidates to collect the maximum amount of Cherenkov light within quartz plates. The current fiber design of the CMS HE calorimeter plastic scintillators collects the scintillation photons from the edges of the plates with WLS fibers. This simple fiber geometry works well for the scintillators since the scintillation photons are generated in random directions. However, the Cherenkov photons have a fixed angle with respect to the momentum of the charged particle. Since Cherenkov photons are already scarce, scattering the photons all the way to the edges would yield small amount of photons. Various fiber embedding geometries were studied at test beams and GEANT4 simulations; Bar-shape, HE-shape, Y-shape, and S-shape, as shown in Figure 4.1. Eventually, the bar-shaped geometry proved to be the best option with the light collection of 
$70 \%$ of the original HE plastic scintillator tile [76].

The calorimeter prototype was prepared with bar-shaped fiber geometry embedded into the wedge shaped grooves of $5 \mathrm{~mm}$ thick GE-124 quartz plates. Polymicro Company grooved the quartz plates. Saint Gobain BCF-12 WLS fibers were inserted into each groove. Each quartz plate - WLS fiber combination was readout via Hamamatsu R7525-HA photomultiplier tubes (PMTs) [78, 79]. These 8 stage, head-on (meaning the active area receives light through one end of the glass increasing uniformity compared to side-on), 1 inch diameter PMTs have a peak quantum efficiency at $425 \mathrm{~nm}$, and are a very good fit for this application. Each layer is a stand-alone unit with $20 \mathrm{~cm}$ x $20 \mathrm{~cm}$ x $0.5 \mathrm{~cm}$ quartz plate, embedded WLS fibers, and a Hamamatsu R7525 PMT. The quartz plate and fiber combination is wrapped with aluminized Mylar for specular reflection. Afterwards the whole unit was wrapped with Tyvek for light tightness. By changing the absorber thickness the prototype was utilized as an Electromagnetic (EM) and Hadronic calorimeter. In the EM configuration, the iron absorber thickness was to $2 \mathrm{~cm}$. For the Hadronic configuration $7 \mathrm{~cm}$ thick iron absorbers were used.

\subsection{Prototype Testing}

The calorimeter prototype was tested at Cern H2 test beam facility. Pion beams with energies of $20 \mathrm{GeV}, 50 \mathrm{GeV}, 80 \mathrm{GeV}, 100 \mathrm{GeV}, 150 \mathrm{GeV}, 200 \mathrm{GeV}, 300$ $\mathrm{GeV}$, and $350 \mathrm{GeV}$ were used during the hadronic calorimeter tests. The prototype's response to a $300 \mathrm{GeV}$ pion beam can be seen in Figure 4.3. The EM configuration was tested with $20 \mathrm{GeV}, 50 \mathrm{GeV}, 80 \mathrm{GeV}$, and $100 \mathrm{GeV}$ electron beam. The 100 $\mathrm{GeV}$ electron response of the prototype is shown in Figure 4.4. During each run the PMT gain was set to $10^{6}(1500 \mathrm{~V})$. On each case the total calorimeter response was constructed by adding the signal from all individual layers. The signal is also corrected for PMT gain differences and ADC pedestals. The GEANT4 simulation of 


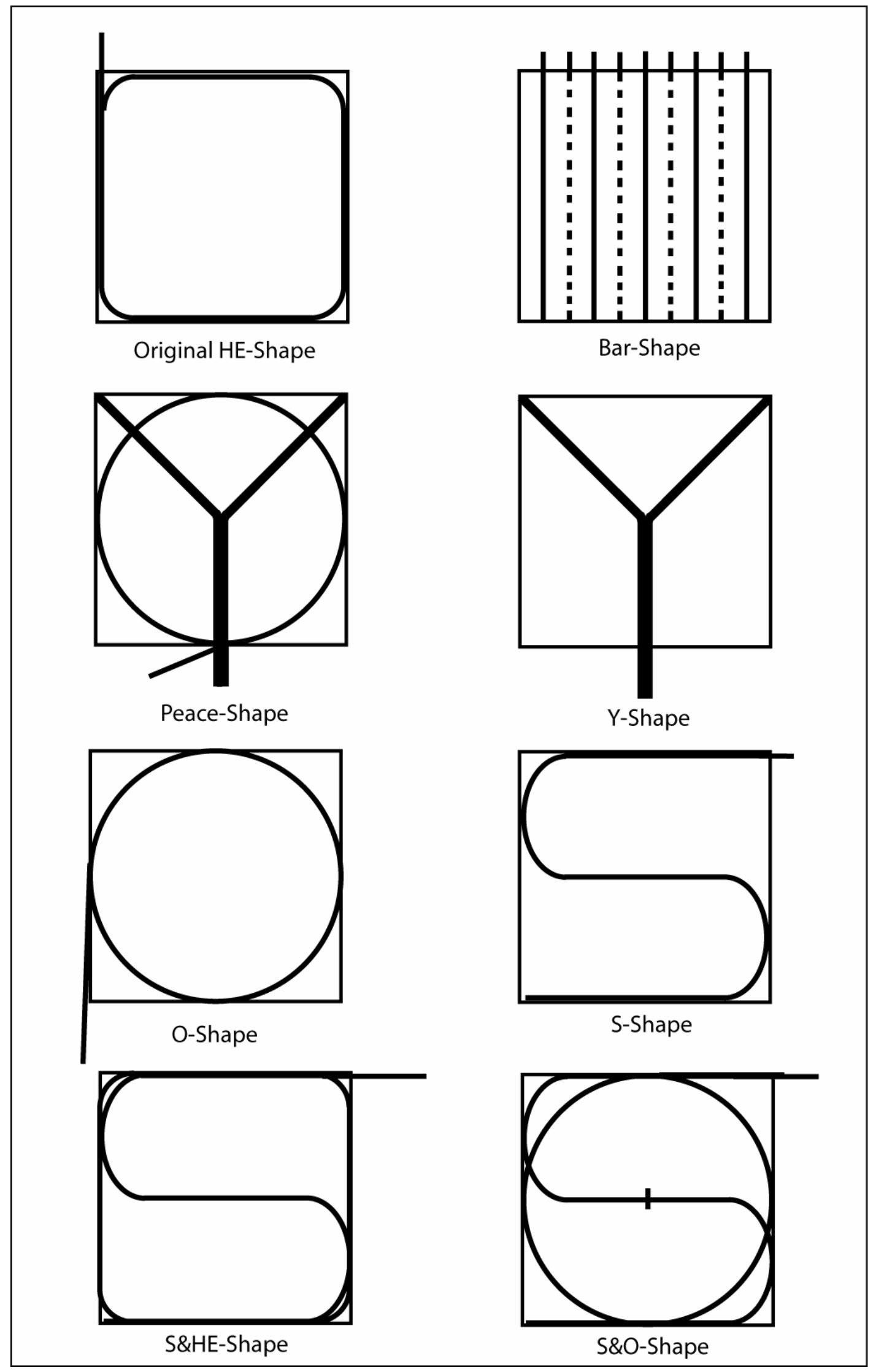

Figure 4.1: Different fiber geometries on plates. 
the model, Figure 4.2, utilized the LHEP physics package. The simulation model counted every photon reaching to the PMT surface. In order to find the resolution the data were fit to the following function:

$$
\frac{\sigma(E)}{E}=\frac{A}{\sqrt{E}}+\frac{B}{E}+C
$$

where $\mathrm{A}$ is the stochastic term, $\mathrm{B}$ is the noise term, and $\mathrm{C}$ is the constant term. The hadronic resolution yields a $235 \pm 4 \%$ stochastic term, negligible $(0.03 \%)$ noise term and $10.9 \pm 0.4 \%$ constant term (see Figure 4.5 ). The calorimeter prototype hadronic response linearity is within $0.1 \%$ up to $350 \mathrm{GeV}$ pion energy (see Figure 4.6). The electromagnetic configuration resolution (see Figure 4.7) fit yields a stochastic term of $31 \pm 2$, with noise of $7.5 \pm 0.5$, and a constant term of $6.7 \pm 0.2$. The electromagnetic response linearity of the calorimeter prototype is also found to be better than $1 \%$ (see Figure 4.8).

\subsection{Summary and Future Prospects}

The LHC luminosity is planned to increase in the coming years. The resulting radiation damage problems will require upgrades on many detectors in LHC experiments. For this purpose, it has been proposed to replace the existing scintillators of CMS HE calorimeter with quartz plates. On previous reports two separate upgrade scenarios, based on quartz plates were proposed. The first model uses pTp to improve the light collection on quartz, and reads signal form the edge of the plate [76]. However, this approach requires the light to be collected by a light detector from the edge of the plate. The $9 \mathrm{~mm}$ gap between current HE calorimeter absorbers, the radiation level as well as the high magnetic field strength at HE location requires a special light detector, which is commercially not available. The second approach, which uses UV absorbing WLS fibers eliminates the radiation and magnetic field 


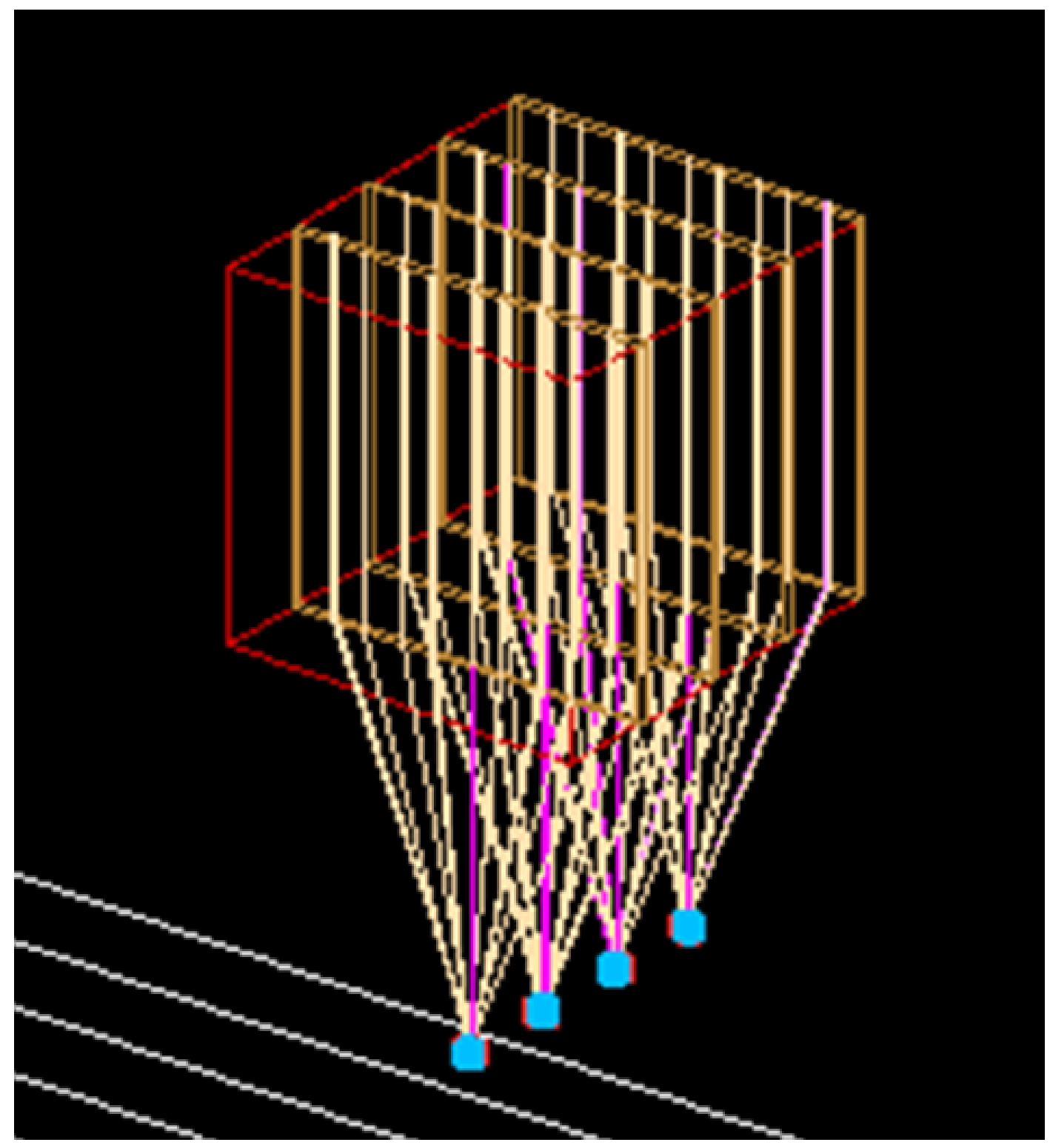

Figure 4.2: This is an image of four of the layers created in the GEANT4 Simulation

problems by carrying the light out of the region. This result shows that by using Saint Gobain BCF-412 WLS fibers, an effective Hadronic and Electromagnetic calorimeter can be built. When considering upgrading the CMS HE calorimeter, the WLS fiber embedded quartz plate calorimeter is a promising option in terms of achieving the current $\mathrm{HE}$ calorimeter performance, which is around $8 \%$ in hadronic 


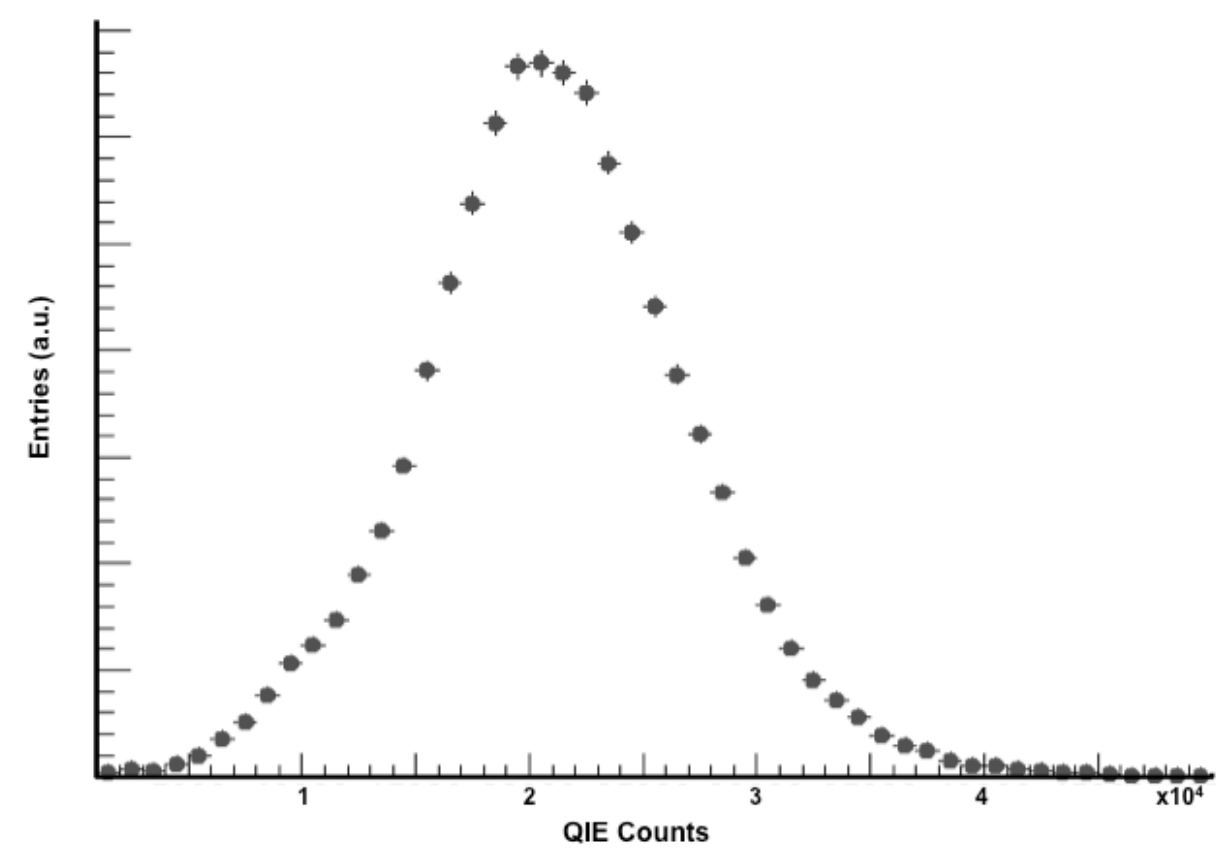

Figure 4.3: The $300 \mathrm{GeV}$ pion response of the calorimeter prototype in the hadronic configuration; where one QIE count corresponds to $2.7 \mathrm{fC}$.

energy resolution at a $300 \mathrm{GeV}$ pion beam energy. Considering the Endcap Electromagnetic (EE) calorimeters, which are located in front of the HE, are exposed to the same radiation levels, quartz plates can be answer for the entire EndCap region. However, the WLS fibers used in this study are only moderately radiation hard $[80,81]$ and cannot be a long-term solution for the future of the LHC. Therefore, the success of this scenario depends on developing radiation hard WLS fiber, which will shape the future of this R\&D study. A radiation hard WLS fiber prototype has been recently built by using quartz fibers and pTp, both known to be radiation hard $[73,74,75]$. 


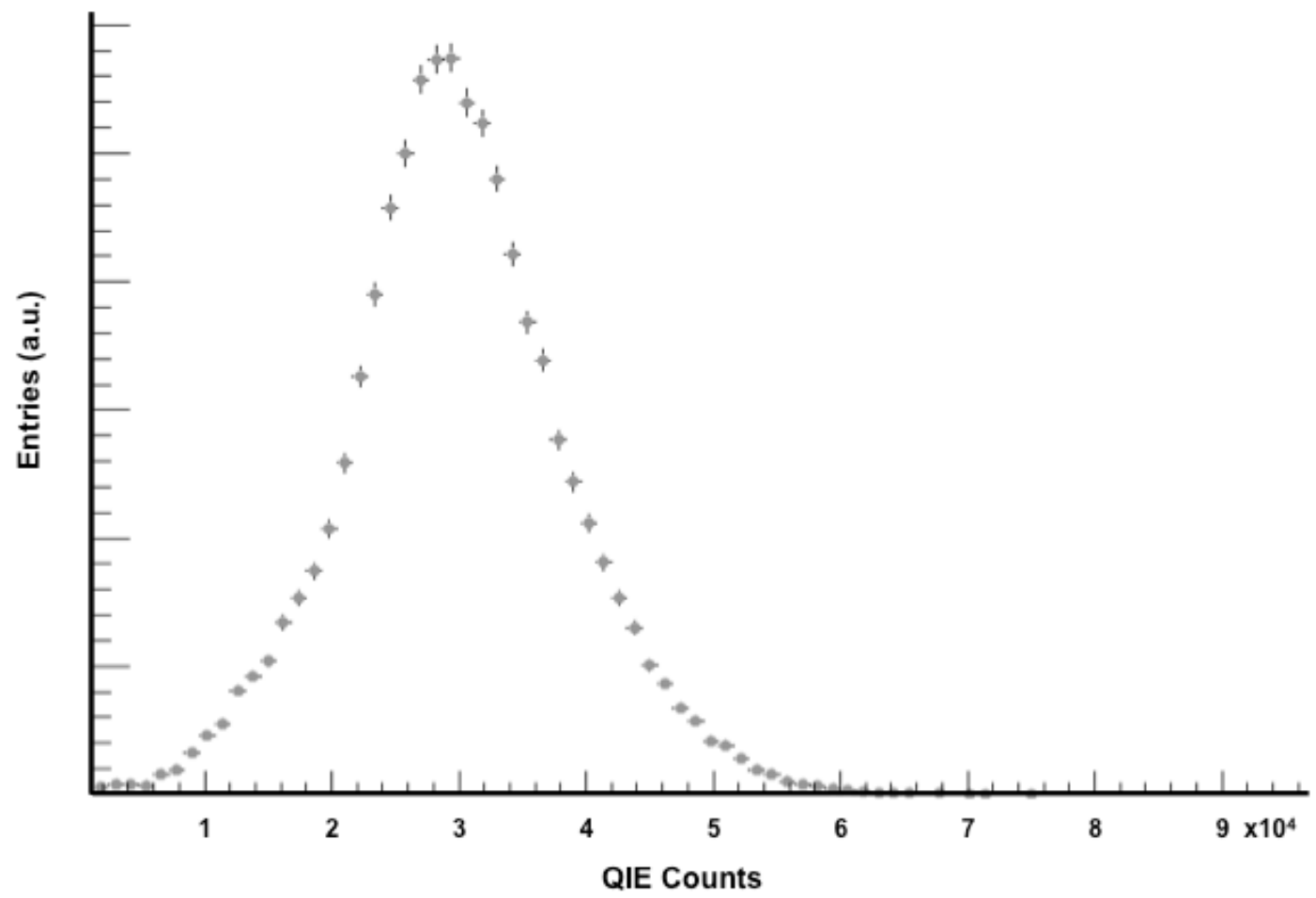

Figure 4.4: The $100 \mathrm{GeV}$ electron response of the calorimeter prototype in the EM configuration; where one QIE count corresponds to $2.7 \mathrm{fC}$. 


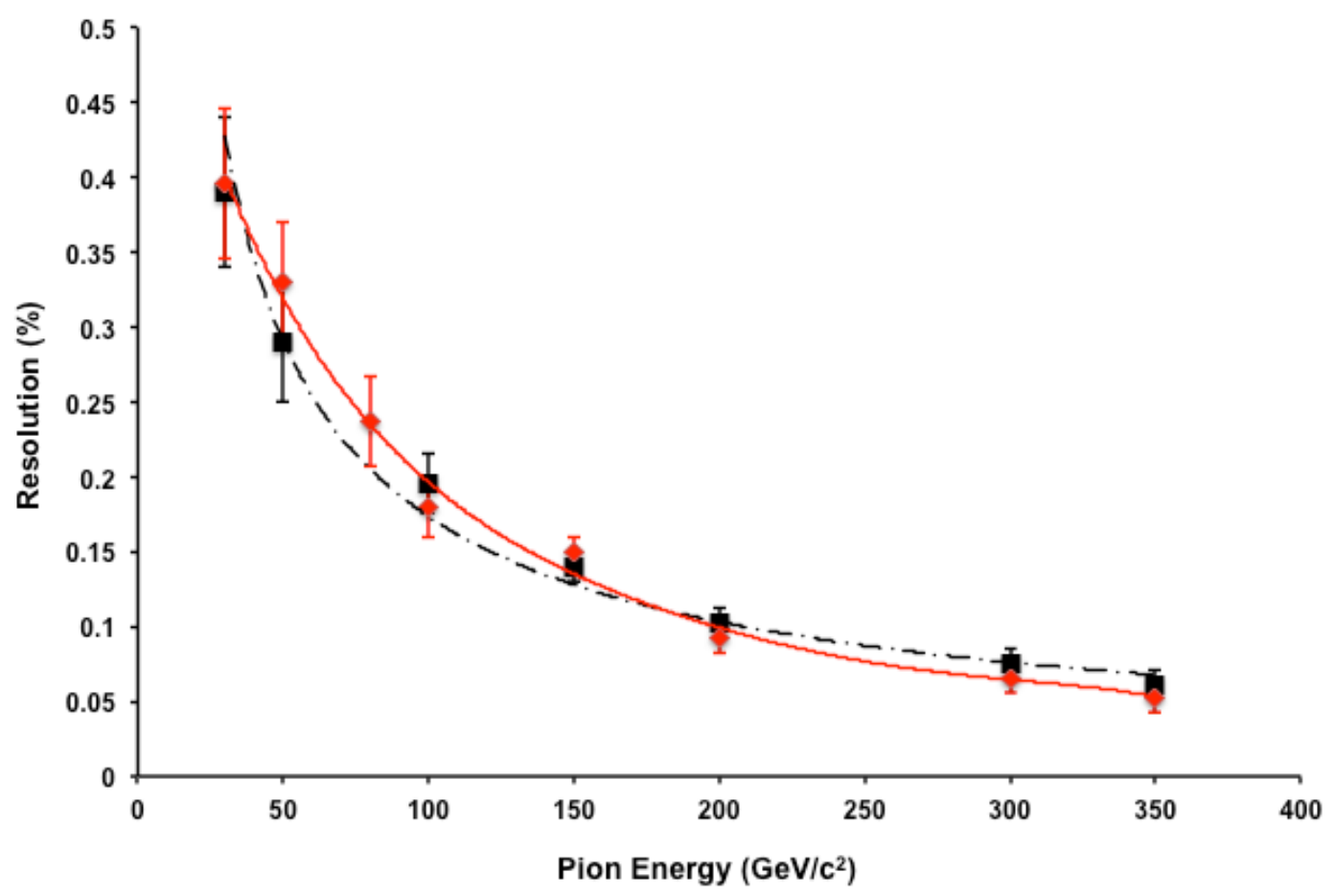

Figure 4.5: The hadronic resolution and statistical error of the quartz plate calorimeter prototype is shown for data (solid red line - circles) and GEANT4 simulations (black dashed line - squares). 


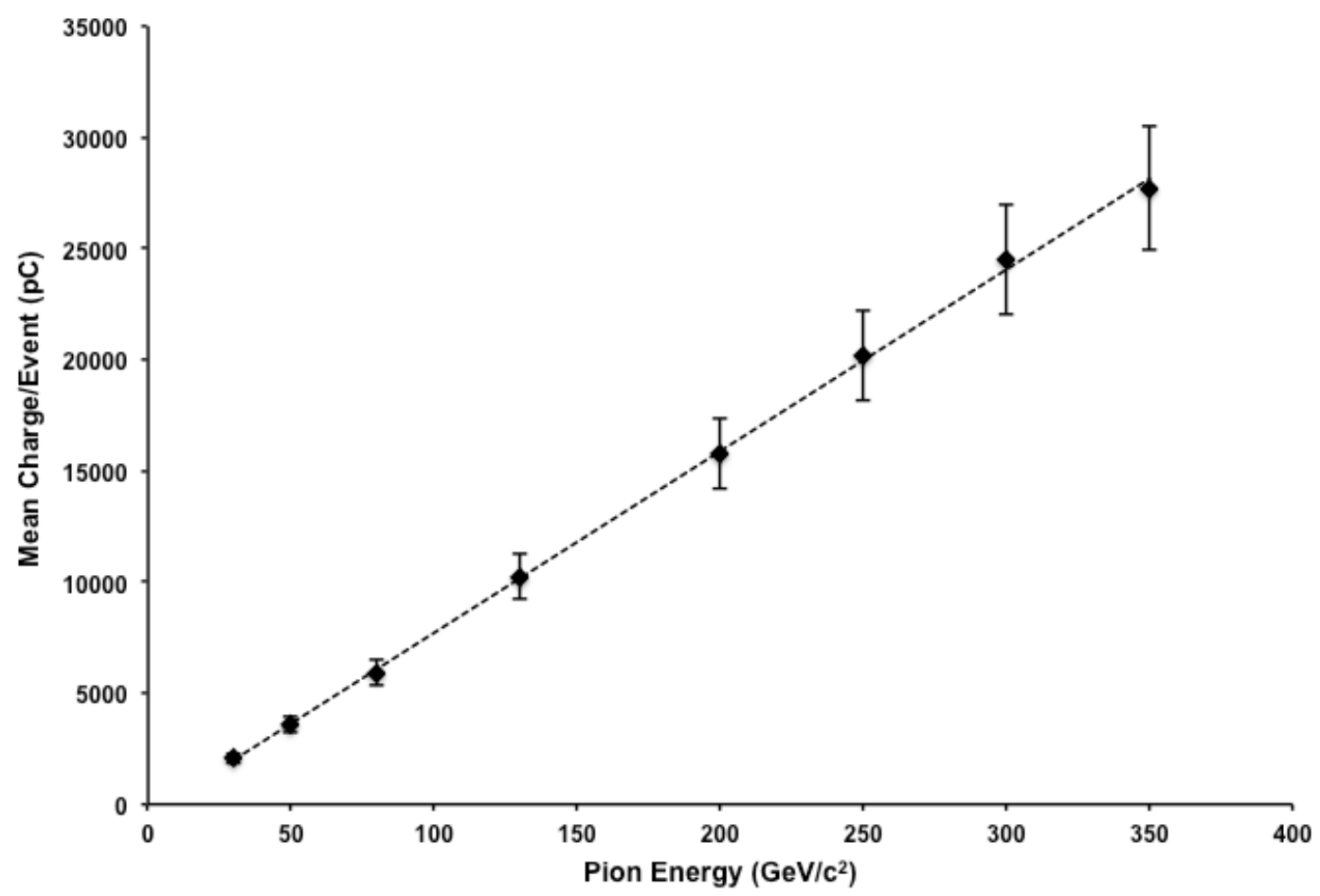

Figure 4.6: The hadronic linearity and statistical error of the quartz plate calorimeter prototype with a coefficient of determination, $R^{2}$, from the fit of 0.99929 .

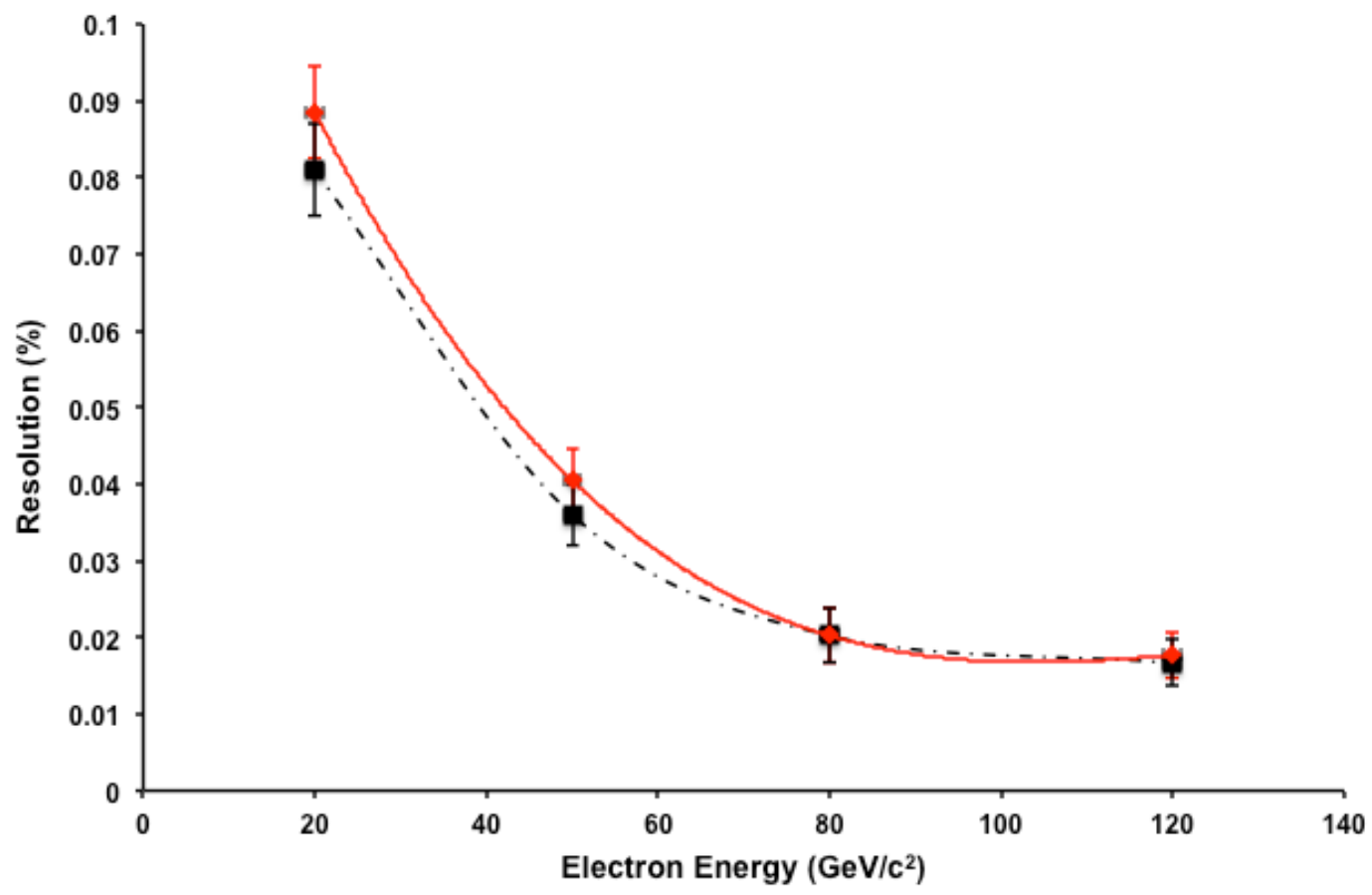

Figure 4.7: The electromagnetic resolution and statistical error of the quartz plate calorimeter prototype is shown for data (solid red line - circles) and GEANT4 simulations (black dashed line - squares). 


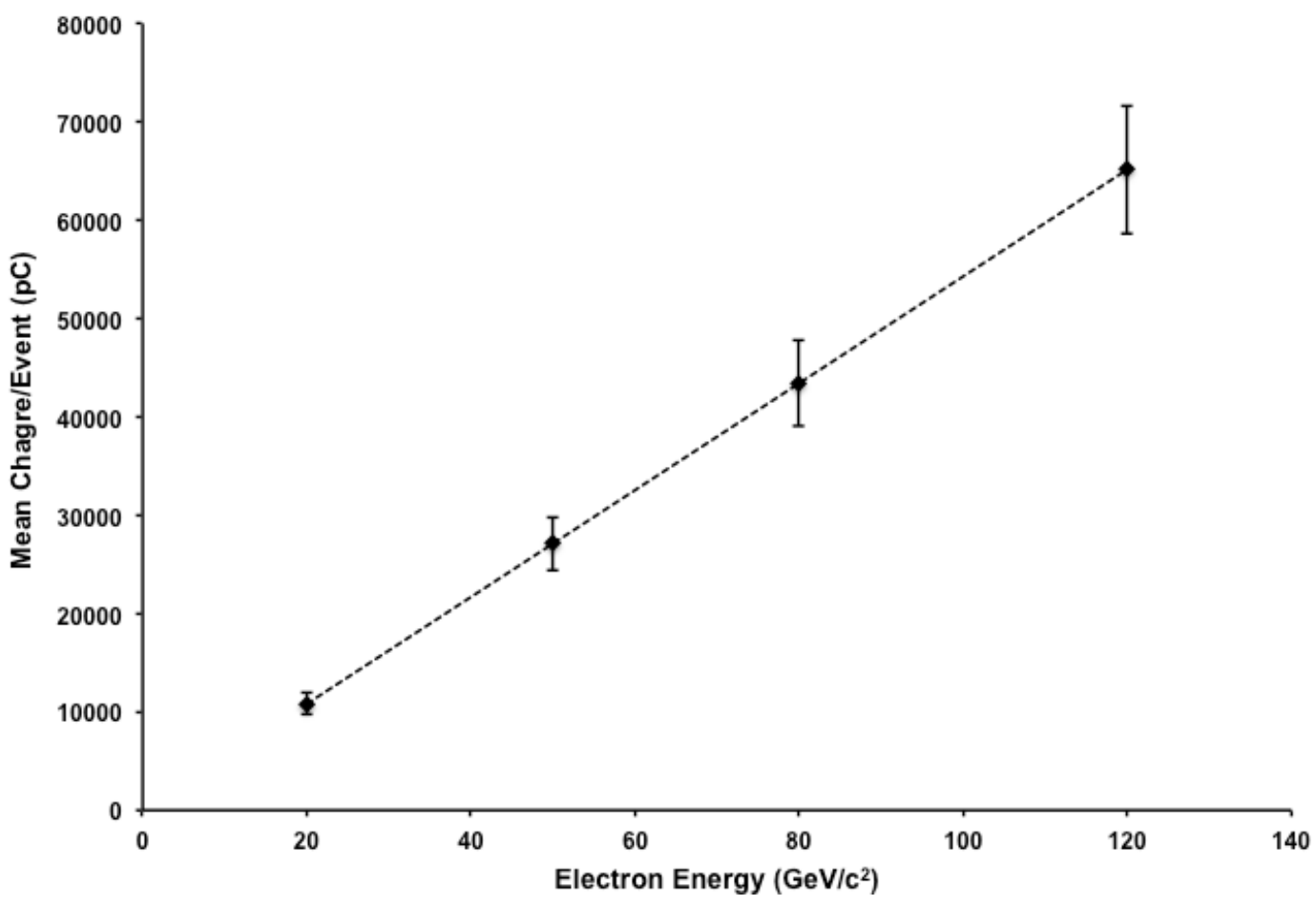

Figure 4.8: The electromagnetic linearity and statistical error of the quartz plate calorimeter prototype; in this case with an $R^{2}$ from the fit of 0.99999 . 


\section{REFERENCES}

[1] D. Griffiths. Introduction to Elementary Particles. WILEY-VCH, Weinheim, Germany, 2008.

[2] R.N. Mohapatra and P.B. Pal. Massive Neutrinos in Physics and Astrophysics. World Scientific Publishing Company, 2004.

[3] M. Fukugita and T. Yanagida. Physics of neutrinos and applications to astrophysics. Texts and monographs in physics. Springer, 2003.

[4] V. Barger, D. Marfatia, and K. Whisnant. "Progress in the physics of massive neutrinos". International Journal of Modern Physics E, 12(05):569-647, 2003.

[5] A. Y. Smirnov. "Neutrino mass and New physics". Journal of Physics: Conference Series, 53(1):44, 2006.

[6] P. Minkowski. " $\mu \rightarrow e \gamma$ at a rate of one out of $10^{9}$ muon decays?". Physics Letters B, 67:421-428, April 1977.

[7] M. Gell-Mann, P. Ramond, and R. Slansky. In P.V. Nieuwenhuizen and D.Z. Freedman, editors, Supergravity: proceedings of the Supergravity Workshop at Stony Brook, page 315. North-Holland, 1979.

[8] T. Yanagida. In O. Sawada and A. Sugamoto, editors, Proceedings of the Workshop on the Unified Theory and the Baryon Number in the Universe, page 95. National Labratory for High Energy Physics (KEK), 1979.

[9] R. N. Mohapatra and G. Senjanović. "Neutrino mass and spontaneous parity nonconservation". Phys. Rev. Lett., 44:912-915, Apr 1980.

[10] W. Pauli. Letter to "Tübigngen Conference". December 4 1930. Translated in L.M. Brown, Phys. Today 23, Sep 1978.

[11] E. Fermi. Z. Phys, 88, 1934. Translated in F.L. Wilson, Am. J. Phys. 36, 1150 (1960).

[12] C. L. Cowan, F. Reines, F. B. Harrison, H. W. Kruse, and A. D. McGuire. Detection of the free neutrino: a confirmation. Science, 124(3212):103-104, 1956. 
[13] B.T. Cleveland et al. Update on the measurement of the solar neutrino flux with the Homestake chlorine detector. Nuclear Physics B - Proceedings Supplements, 38(13):47 - 53, 1995.

[14] R. Davis. A review of the Homestake solar neutrino experiment. Progress in Particle and Nuclear Physics, 32(0):13 - 32, 1994.

[15] J. Beringer et al. "Review of Particle Physics". Phys. Rev. D, 86(010001), 2012.

[16] W.C. Haxton. The Solar-Neutrino Problem. Annual Review of Astronomy and Astrophysics, 33:459-503, 1995.

[17] V. Gribov and B. Pontecorvo. "Neutrino astronomy and lepton charge". Physics Letters B, 28(7):493 - 496, 1969.

[18] Y. Fukuda et al. "Measurement of the flux and zenith-angle distribution of upward throughgoing muons by Super-Kamiokande". Phys. Rev. Lett., 82:26442648, Mar 1999.

[19] Z. Maki, M. Nakagawa, and S. Sakata. "Remarks on the unified model of elementary particles". Progress of Theoretical Physics, 28(5):870-880, 1962.

[20] B. Pontecorvo. "Neutrino experiments and the problem of conservation of leptonic charge". Soviet Journal of Experimental and Theoretical Physics, 26:984, May 1968.

[21] Q. R. Ahmad et al. "Measurement of the rate of $\nu_{e}+d \rightarrow p+p+e^{-}$interactions produced by ${ }^{8} b$ solar neutrinos at the sudbury neutrino observatory". Phys. Rev. Lett., 87:071301, Jul 2001.

[22] The accelerator complex. http://public.web.cern.ch/public/en/research/ AccelComplex-en.html.

[23] L. Evans and P. Bryant. "LHC Machine". Journal of Instrumentation, 3(08):S08001, 2008 .

[24] CMS Collaboration. "The CMS experiment at the CERN LHC". JINST, 3, 2008 .

[25] D. Barney and S. Cittolin. CMS Detector. https://cms-docdb.cern.ch/ cgi-bin/PublicDocDB/ShowDocument?docid=2716, July 2010. 
[26] M.C. Fouz. "The CMS muon system". Nuclear Instruments and Methods in Physics Research Section A: Accelerators, Spectrometers, Detectors and Associated Equipment, 573(12):260 - 263, 2007. Proceedings of the 7th International Conference on Position-Sensitive Detectors.

[27] E. Majorana. "Teoria simmetrica dellelettrone e del positrone". Nuovo Cimento, 9, 1937.

[28] W. Keung and G. Senjanović. "Majorana Neutrinos and the Production of the Right-Handed Charged Gauge Boson". Phys. Rev. Lett., 50:1427-1430, May 1983.

[29] D. A. Dicus, D. D. Karatas, and P. Roy. "Lepton nonconservation at supercollider energies". Phys. Rev. D, 44:2033-2037, Oct 1991.

[30] A. Datta, M. Guchait, and A. Pilaftsis. "Probing lepton number violation via Majorana neutrinos at hadron supercolliders". Phys. Rev. D, 50:3195-3203, Sep 1994.

[31] F. M. L. Almeida, Y. A. Coutinho, J. A. Martins Simões, and M. A. B. do Vale. "Signature for heavy Majorana neutrinos in hadronic collisions". Phys. Rev. D, 62:075004, Sep 2000.

[32] O. Panella, M. Cannoni, C. Carimalo, and Y. N. Srivastava. "Signals of heavy Majorana neutrinos at hadron colliders". Phys. Rev. D, 65:035005, Jan 2002.

[33] T. Han and B. Zhang. "Signatures for Majorana Neutrinos at Hadron Colliders". Phys. Rev. Lett., 97:171804, Oct 2006.

[34] F. del Aguila, J. A. Aguilar-Saavedra, and R. Pittau. "Heavy neutrino signals at large hadron colliders". Journal of High Energy Physics, 2007(10):047, 2007.

[35] A. Atre, T. Han, S. Pascoli, and B. Zhang. "The search for heavy Majorana neutrinos". Journal of High Energy Physics, 2009(05):030, 2009.

[36] P. Abreu et al. "Search for neutral heavy leptons produced in Z decays". Z.Phys., C74:57-71, 1997.

[37] O. Adriani et al. "Search for isosinglet neutral heavy leptons in Z0 decays". Physics Letters B, 295(34):371 - 382, 1992.

[38] T. Sjöstrand, S. Mrenna, and P. Skands. "PYTHIA 6.4 physics and manual". Journal of High Energy Physics, 2006(05):026, 2006. 
[39] M. L. Mangano, F. Piccinini, A. D. Polosa, M. Moretti, and R. Pittau. "ALPGEN, a generator for hard multiparton processes in hadronic collisions". Journal of High Energy Physics, 2003(07):001, 2003.

[40] F. Caravaglios and M. Moretti. "An algorithm to compute Born scattering amplitudes without Feynman graphs". Physics Letters B, 358(3-4):332 - 338, 1995 .

[41] Hung-Liang Lai et al. "New parton distributions for collider physics". Phys.Rev., D82:074024, 2010.

[42] The Durham HEPData Project. http://hepdata.cedar.ac.uk/pdf/pdf3. html.

[43] S. Agostinelli et al. "Geant4-A simulation toolkit". Nuclear Instruments and Methods in Physics Research Section A: Accelerators, Spectrometers, Detectors and Associated Equipment, 506(3):250 - 303, 2003.

[44] CMS Collaboration. "Performance of CMS muon identification in $p p$ collisions at $\sqrt{s}=7 \mathrm{TeV} "$. CMS PAS, MOU-10-002, 2010.

[45] M. Cacciari, G. P. Salam, and G. Soyez. "The anti- $k_{t}$ jet clustering algorithm". Journal of High Energy Physics, 2008(04):063, 2008.

[46] Y.L. Dokshitzer, G.D. Leder, S. Moretti, and B.R. Webber. Better jet clustering algorithms. Journal of High Energy Physics, 1997(08):001, 1997.

[47] CMS Collaboration. "Determination of jet energy calibration and transverse momentum resolution in CMS". JINST, 6, 2011.

[48] M.R. Whalley, D. Bourilkov, and R.C. Group. "The Les Houches accord PDFs (LHAPDF) and Lhaglue". August 2005. http://arxiv.org/abs/hep-ph/ 0508110 .

[49] CMS Collaboration. "Search for a Standard Model Higgs boson produced in the decay channel 4l". CMS PAS, HIG-11-004, 2011.

[50] W. Andrews et al. "Search for higgs boson decay to two w bosons in the fully leptonic final state at $\sqrt{7} \mathrm{TeV}$ with 2011 data with the CMS detector". CMS Note, AN-2011-147, 2011.

[51] D. Barge et al. "Inclusive search for new physics with same-sign dileptons using early LHC data". CMS Note, AN-2010-247, 2010. 
[52] D. Barge et al. "Search for new physics with same-sign dileptons using the 2011 dataset of CMS". CMS Note, AN-2011-258, 2011.

[53] G. Bauer et al. "Lepton effiencies for the inclusive w cross section measurement with 36.1 $\mathrm{pb}^{-1} "$. CMS Note, AN-2011-097, 2011.

[54] D. Barge et al. "Fake rates for dilepton analyses". CMS Note, AN-2010-257, 2010 .

[55] CMS Collaboration. "Search for heavy Majorana neutrinos in $\mu^{ \pm} \mu^{ \pm}$and $e^{ \pm} e^{ \pm}$ events in pp collisions at $\sqrt{s}=7 \mathrm{TeV}$ ". Physics Letters B, 717(13):109 - 128, 2012 .

[56] D Bourilkov, R C Group, and M R Whalley. "LHAPDF: PDF use from the Tevatron to the LHC". 2006. http://arxiv.org/abs/hep-ph/0605240.

[57] J. Berger et al. "Absolute jet energy correction and its uncertainty with $36 \mathrm{pb}^{-1} "$. CMS Note, AN-2011/105, 2011.

[58] Estimating systematic errors due to pileup modeling. https://twiki.cern. ch/twiki/bin/view/CMS/PileupSystematicErrors.

[59] The CMS Collaboration. "Performance of CMS muon reconstruction in pp collisions at $\sqrt{s}=7 \mathrm{TeV}$ ". CMS PAS, CMS-PAS-MOU-10-004, 2010.

[60] A.L. Read. "Presentation of search results: the CLs technique". Journal of Physics G: Nuclear and Particle Physics, 28, 2002.

[61] M. Lorenzo et al. "The RooStats Project". PoS, ACAT2010:057, 2010.

[62] RooStatscl95: "Cross Section in a Counting Experiment". https://twiki. cern.ch/twiki/bin/viewauth/CMS/RooStatsCl95.

[63] E. Nardi, E. Roulet, and D. Tommasini. "New neutral gauge bosons and new heavy fermions in the light of the new LEP data". Physics Letters B, 344(14):225 - 232, 1995.

[64] W. Scandale and F. Zimmermann. "Scenarios for SLHC and vLHC". Nucl. Phys. B (Proc. Suppl.), pages 177-178, 207-211, 2008.

[65] M. Huhtinen. "The radiation enviroment at the CMS experiment at the LHC". Ph.D Thesis, 1996.

[66] S. Abdullin et al. "Physics potential and experimental challenges of the LHC luminosity upgrade". 2002. http://arxiv.org/abs/hep-ph/0204087. 
[67] I. Golutvin et al. "Simulation of radiation damage in HE scintillating tiles and pion energy resolution after 10 years of LHC operation". CMS-Note, 2002/013, 2002.

[68] G. Baiatian et al. "Design, Performance, and Calibration of CMS Hadron Endcap Calorimeters". CMS-Note, 2008/010, 2008.

[69] CMS Collaboration. "CMS Physics TDR: Volume I, Detector Performance and Software". CERN/LHCC, 2006-001, 2006.

[70] U. Akgun et al. "Quartz Plate Calorimeter as SLHC Upgrade to CMS Hadronic Endcap Calorimeters". XIII International Conference on Calorimetry in High Energy Physics, CALOR 2008, 2008.

[71] U. Akgun and Y. Onel. "Radiation-Hard Quartz Cerenkov Calorimeters". AIP Conf. Proc, 867:282-289, 2006.

[72] B. Bilki et al. "CMS Hadron Endcap Calorimeter Upgrade Studies for SuperLHC". Beijing, China, 2010.

[73] I. Dumanoglu et al. "Radiation-hardness Studies of High-OH Content Fibers Irradiated with $500 \mathrm{MeV}$ Electrons". Nucl. Instrum. Meth A, 490:444-455, 2002.

[74] K. Cankocak et al. "Radiation-hardness Measurements of High-OH Content Fibers Irradiated with $24 \mathrm{GeV}$ Protons up to 1.25 Grad". Nucl. Instrum. Meth A, 490:20-27, 2008.

[75] U. Akgun et al. "CMS Hadronic Endcap Calorimeter Upgrade Studies for SLHC, P-Terphenyl Deposited Quartz Plate Calorimeter Prototype". IEEE Trans. Nucl. Sci., 57:754-759, 2010.

[76] F. Duru et al. "CMS Hadronic EndCap Calorimeter Upgrade Studies for SLHC - Cerenkov Light Collection from Quartz Plates". IEEE Trans. Nucl. Sci., 55:734-740, 2008.

[77] Saint Gobain crystals. http://www.detectors.saint-gobain.com.

[78] U. Akgun et al. "Comparison of PMTs from three different manufactures for the CMS-HF Forward Calorimeter". IEEE Trans. Nucl. Sci., 51:1909-1915, 2004.

[79] U. Akgun et al. "Complete Tests of 2000 Hamamatsu R7525HA Phototubes for the CMS-HF Forward Calorimeter". Nucl. Instrum. Meth. A, 550:145-156, 2005 . 
[80] W. Busjan, K. Wick, and T. Zoufal. "On The Behaviour of Plastic Scintillators During an Experiment in a High Dose Rate Environment". Nucl. Instrum. Meth. B, 151:434-437, 1999.

[81] W. Busjan, K. Wick, and T. Zoufal. "Shortlived Absorption Centers in Plastic Scintillators and Their Influence on the Flourescence Light Yield". Nucl Instrum. Meth. B, 152:89-104, 1999. 